\title{
Financial Time Series Forecasting with Deep Learning : A Systematic Literature Review: 2005-2019
}

\author{
Omer Berat Sezer ${ }^{\mathrm{a}}$, M. Ugur Gudelek ${ }^{\mathrm{a}}$, Ahmet Murat Ozbayoglu \\ ${ }^{a}$ Department of Computer Engineering, TOBB University of Economics and Technology, Ankara, Turkey
}

\begin{abstract}
Financial time series forecasting is, without a doubt, the top choice of computational intelligence for finance researchers from both academia and financial industry due to its broad implementation areas and substantial impact. Machine Learning (ML) researchers came up with various models and a vast number of studies have been published accordingly. As such, a significant amount of surveys exist covering ML for financial time series forecasting studies. Lately, Deep Learning (DL) models started appearing within the field, with results that significantly outperform traditional ML counterparts. Even though there is a growing interest in developing models for financial time series forecasting research, there is a lack of review papers that were solely focused on DL for finance. Hence, our motivation in this paper is to provide a comprehensive literature review on DL studies for financial time series forecasting implementations. We not only categorized the studies according to their intended forecasting implementation areas, such as index, forex, commodity forecasting, but also grouped them based on their DL model choices, such as Convolutional Neural Networks (CNNs), Deep Belief Networks (DBNs), Long-Short Term Memory (LSTM). We also tried to envision the future for the field by highlighting the possible setbacks and opportunities, so the interested researchers can benefit.
\end{abstract}

Keywords: deep learning, finance, computational intelligence, machine learning, time series forecasting, CNN, LSTM, RNN

\section{Introduction}

The finance industry has always been interested in successful prediction of financial time series data. Numerous studies have been published that were based on ML models with relatively better performances compared to classical time series forecasting techniques. Meanwhile, the widespread application of automated electronic trading systems coupled with increasing demand for higher yields keeps forcing the researchers and practitioners to continue working on searching for better models. Hence, new publications and implementations keep pouring into finance and computational intelligence literature.

In the last few years, DL started emerging strongly as the best performing predictor class within the ML field in various implementation areas. Financial time series forecasting is no exception, as such, an increasing number of prediction models based on various DL techniques were introduced in the appropriate conferences and journals in recent years. 
Despite the existence of the vast amount of survey papers covering financial time series forecasting and trading systems using traditional soft computing techniques, to the best of our knowledge, no reviews have been performed in literature for DL. Hence, we decided to work on such a comprehensive study focusing on DL implementations of financial time series forecasting. Our motivation is two-fold such that we not only aimed at providing the state-of-the-art snapshot of academic and industry perspectives of the developed DL models but also pinpointing the important and distinctive characteristics of each studied model to prevent researchers and practitioners to make unsatisfactory choices during their system development phase. We also wanted to envision where the industry is heading by indicating possible future directions.

Our fundamental motivation in this paper was to come up with answers for the following research questions:

- Which DL models are used for financial time series forecasting ?

- How is the performance of DL models compared with traditional ML counterparts ?

- What is the future direction for DL research for financial time series forecasting ?

Our focus was solely on DL implementations for financial time series forecasting. For other DL based financial applications such as risk assessment, portfolio management, etc., interested readers can check the recent survey paper [1]. Since we singled out financial time series prediction studies in our survey, we omitted other time series forecasting studies that were not focused on financial data. Meanwhile, we included time-series research papers that had financial use cases or examples even though the papers themselves were not directly intended for financial time series forecasting. Also, we decided to include algorithmic trading papers that were based on financial forecasting, but ignore the ones that did not have a time series forecasting component.

We reviewed journals and conferences for our survey, however, we also included Masters and $\mathrm{PhD}$ theses, book chapters, arXiv papers and noteworthy technical publications that came up in web searches. We decided to only include the articles in the English language.

During our survey through the papers, we realized that most of the papers using the term "deep learning" in their description were published in the last 5 years. However, we also encountered some older studies that implemented deep models; such as Recurrent Neural Networks (RNNs), Jordan-Elman networks. However, at their time of publication, the term "deep learning" was not in common usage. So, we decided to also include those papers.

According to our findings, this will be one of the first comprehensive "financial time series forecasting" survey papers focusing on DL. A lot of ML reviews for financial time series forecasting exist in the literature, meanwhile, we have not encountered any study on DL. Hence, we wanted to fill this gap by analyzing the developed models and applications accordingly. We hope, as a result of this paper, the researchers and model developers will have a better idea of how they can implement DL models for their studies.

We structured the rest of the paper as follows. Following this brief introduction, in Section 2, the existing surveys that are focused on ML and soft computing studies for financial time series forecasting are mentioned. In Section 3, we will cover the existing DL 
models that are used, such as CNN, LSTM, Deep Reinforcement Learning (DRL). Section 4 will focus on the various financial time series forecasting implementation areas using DL, namely stock forecasting, index forecasting, trend forecasting, commodity forecasting, volatility forecasting, foreign exchange forecasting, cryptocurrency forecasting. In each subsection, the problem definition will be given, followed by the particular DL implementations. In Section 5, overall statistical results about our findings will be presented including histograms about the yearly distribution of different subfields, models, publication types, etc. As a result, the state-of-the-art snapshot for financial time series forecasting studies will be given through these statistics. At the same time, it will also show the areas that are already mature, compared against promising or new areas that still have room for improvement. Section 6 will provide discussions about what has been done through academic and industrial achievements and expectations through what might be needed in the future. The section will include highlights about the open areas that need further research. Finally, we will conclude in Section 7 by summarizing our findings.

\section{Financial Time Series Forecasting with ML}

Financial time series forecasting and associated applications have been studied extensively for many years. When ML started gaining popularity, financial prediction applications based on soft computing models also became available accordingly. Even though our focus is particularly on DL implementations of financial time series prediction studies, it will be beneficial to briefly mention about the existing surveys covering ML-based financial time series forecasting studies in order to gain historical perspective.

In our study, we did not include any survey papers that were focused on specific financial application areas other than forecasting studies. However, we were faced with some review publications that included not only financial time-series studies but also other financial applications. We decided to include those papers in order to maintain the comprehensiveness of our coverage.

Examples of these aforementioned publications are provided here. There were published books on stock market forecasting [2], trading system development [3], practical examples of forex and market forecasting applications [4] using ML models like Artificial Neural Networks (ANNs), Evolutionary Computations (ECs), Genetic Programming (GP) and Agent-based models [5].

There were also some existing journal and conference surveys. Bahrammirzaee et. al. [6] surveyed financial prediction and planning studies along with other financial applications using various Artificial Intelligence (AI) techniques like ANN, Expert Systems, hybrid models. The authors of [7] also compared ML methods in different financial applications including stock market prediction studies. In [8], soft computing models for the market, forex prediction and trading systems were analyzed. Mullainathan and Spies [9] surveyed the prediction process in general from an econometric perspective.

There were also a number of survey papers concentrated on a single particular ML model. Even though these papers focused on one technique, the implementation areas generally 
spanned various financial applications including financial time series forecasting studies. Among those soft computing methods, EC and ANN had the most overall interest.

For the EC studies, Chen wrote a book on Genetic Algorithms (GAs) and GP in Computational Finance [10]. Later, Multiobjective Evolutionary Algorithms (MOEAs) were extensively surveyed on various financial applications including financial time series prediction $[11,12,13]$. Meanwhile, Rada reviewed EC applications along with Expert Systems for financial investing models [14].

For the ANN studies, Li and Ma reviewed implementations of ANN for stock price forecasting and some other financial applications [15]. The authors of [16] surveyed different implementations of ANN in financial applications including stock price forecasting. Recently, Elmsili and Outtaj contained ANN applications in economics and management research including economic time series forecasting in their survey [17].

There were also several text mining surveys focused on financial applications (which included financial time series forecasting). Mittermayer and Knolmayer compared various text mining implementations that extract market response to news for prediction [18]. The authors of [19] focused on news analytics studies for prediction of abnormal returns for trading strategies in their survey. Nassirtoussi et. al. reviewed text mining studies for stock or forex market prediction [20]. The authors of [21] also surveyed text mining-based time series forecasting and trading strategies using textual sentiment. Similarly, Kumar and Ravi [22] reviewed text mining studies for forex and stock market prediction. Lately, Xing et. al. [23] surveyed natural language-based financial forecasting studies.

Finally, there were application-specific survey papers that focused on particular financial time series forecasting implementations. Among these studies, stock market forecasting had the most interest. A number of surveys were published for stock market forecasting studies based on various soft computing methods at different times [24, 25, 26, 27, 28, 29, 30, 31]. Chatterjee et. al. [32] and Katarya and Mahajan [33] concentrated on ANN-based financial market prediction studies whereas $\mathrm{Hu}$ et. al. [34] focused on EC implementations for stock forecasting and algorithmic trading models. In a different time series forecasting application, researchers surveyed forex prediction studies using ANN [35] and various other soft computing techniques [36].

Even though, many surveys exist for ML implementations of financial time series forecasting, DL has not been surveyed comprehensively so far despite the existence of various DL implementations in recent years. Hence, this was our main motivation for the survey. At this point, we would like to cover the various DL models used in financial time series forecasting studies.

\section{Deep Learning}

DL is a type of ANN that consists of multiple processing layers and enables high-level abstraction to model data. The key advantage of DL models is extracting the good features of input data automatically using a general-purpose learning procedure. Therefore, in the literature, DL models are used in lots of applications: image, speech, video, audio reconstruction, natural language understanding (particularly topic classification), sentiment 
analysis, question answering and language translation [37]. The historical improvements on DL models are surveyed in [38].

Financial time series forecasting has been very popular among ML researchers for more than 40 years. The financial community got a new boost lately with the introduction of DL models for financial prediction research and a lot of new publications appeared accordingly. The success of DL over ML models is the major attractive point for the finance researchers. With more financial time series data and different deep architectures, new DL methods will be proposed. In our survey, we found that in the vast majority of the studies, DL models were better than ML counterparts.

In literature, there are different kinds of DL models: Deep Multilayer Perceptron (DMLP), RNN, LSTM, CNN, Restricted Boltzmann Machines (RBMs), DBN, Autoencoder (AE), and DRL [37, 38]. Throughout the literature, financial time series forecasting was mostly considered as a regression problem. However, there were also a significant number of studies, in particular trend prediction, that used classification models to tackle financial forecasting problems. In Section 4, different DL implementations are provided along with their model choices.

\subsection{Deep Multi Layer Perceptron (DMLP)}

DMLPs is one of the first developed ANNs. The difference from shallow nets is that DMLP contains more layers. Even though particular model architectures might have variations depending on different problem requirements, DMLP models consist of mainly three layers: input, hidden and output. The number of neurons in each layer and the number of layers are the hyperparameters of the network. In general, each neuron in the hidden layers has input $(x)$, weight $(w)$ and bias $(b)$ terms. In addition, each neuron has a nonlinear activation function which produces a cumulative output of the preceding neurons. Equation 1 [39] illustrates an output of a single neuron in the Neural Network (NN). There are different types of nonlinear activation functions. Most commonly used nonlinear activation functions are: sigmoid (Equation 2) [40], hyperbolic tangent (Equation 3) [41], Rectified Linear Unit (ReLU) (Equation 4) [42], leaky-ReLU (Equation 5) [43], swish (Equation 6) [44], and softmax (Equation 7) [39]. The comparison of the nonlinear activations are studied in [44].

$$
\begin{gathered}
y_{i}=\sigma\left(\sum_{i} W_{i} x_{i}+b_{i}\right) \\
\sigma(z)=\frac{1}{1+e^{-z}} \\
\tanh (z)=\frac{e^{z}-e^{-z}}{e^{z}+e^{-z}} \\
R(z)=\max (0, z) \\
R(z)=1(x<0)(\alpha x)+1(x>=0)(x)
\end{gathered}
$$




$$
\begin{gathered}
f(x)=x \sigma(\beta x) \\
\operatorname{softmax}\left(z_{i}\right)=\frac{\exp z_{i}}{\sum_{j} \exp z_{j}}
\end{gathered}
$$

DMLP models have been appearing in various application areas [45, 37]. Using a DMLP model has advantages and disadvantages depending on the problem requirements. Through DMLP models, problems such as regression and classification can be solved by modeling the input data [46]. However, if the number of the input features is increased (e.g. image as input), the parameter size in the network will increase accordingly due to the fully connected nature of the model and it will jeopardize the computation performance and create storage problems. To overcome this issue, different types of Deep Neural Network (DNN) methods are proposed (such as CNN) [37]. With DMLP, much more efficient classification and regression processes are performed. In Figure 1, a DMLP model, layers, neurons in layers, weights between neurons are shown.

Forward Pass

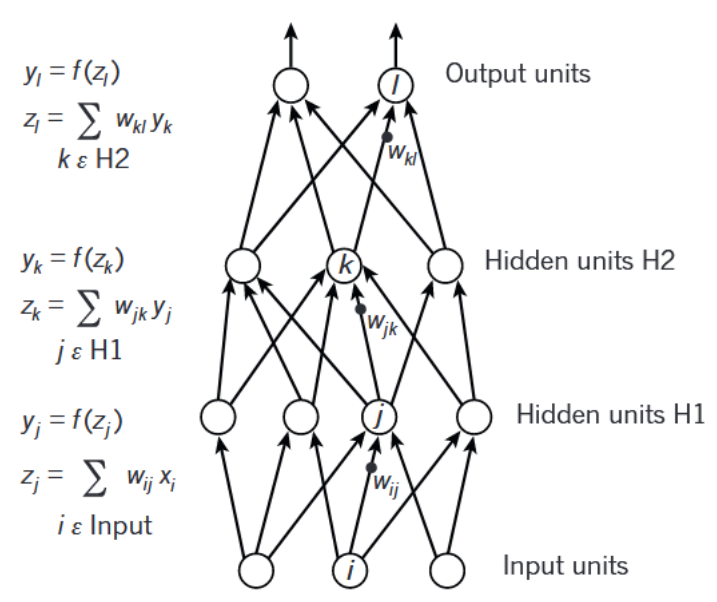

Backpropagation



Figure 1: Deep Multi Layer Neural Network Forward Pass and Backpropagation [37]

DMLP learning stage is implemented through backpropagation. The amount of error in the neurons in the output layer is propagated back to the preceeding layers. Optimization algorithms are used to find the optimum parameters/variables of the NNs. They are used to update the weights of the connections between the layers. There are different optimization algorithms that are developed: Stochastic Gradient Descent (SGD), SGD with Momentum, Adaptive Gradient Algorithm (AdaGrad), Root Mean Square Propagation (RMSProp), Adaptive Moment Estimation (ADAM) [47, 48, 49, 50, 51]. Gradient descent is an iterative method to find optimum parameters of the function that minimizes the cost function. SGD is an algorithm that randomly selects a few samples instead of the whole data 
set for each iteration [47]. SGD with Momentum remembers the update in each iteration that accelerates gradient descent method [48]. AdaGrad is a modified SGD that improves convergence performance over standard SGD algorithm [49]. RMSProp is an optimization algorithm that provides the adaptation of the learning rate for each of the parameters. In RMSProp, the learning rate is divided by a running average of the magnitudes of recent gradients for that weight [50]. ADAM is updated version of RMSProp that uses running averages of both the gradients and the second moments of the gradients. ADAM combines advantages of the RMSProp (works well in online and non-stationary settings) and AdaGrad (works well with sparse gradients) [51].

As shown in Figure 1, the effect of the backpropagation is transferred to the previous layers. If the effect of SGD is gradually lost when the effect reaches the early layers during backpropagation, this problem is called vanishing gradient problem in the literature [52]. In this case, updates between the early layers become unavailable and the learning process stops. The high number of layers in the neural network and the increasing complexity cause the vanishing gradient problem.

The important issue in the DMLP are the hyperparameters of the networks and method of tuning these hyperparameters. Hyperparameters are the variables of the network that affect the network architecture, and the performance of the networks. The number of hidden layers, the number of units in each layer, regularization techniques (dropout, L1, L2), network weight initialization (zero, random, He [53], Xavier [54]), activation functions (Sigmoid, ReLU, hyperbolic tangent, etc.), learning rate, decay rate, momentum values, number of epochs, batch size (minibatch size), and optimization algorithms (SGD, AdaGrad, RMSProp, ADAM, etc.) are the hyperparameters of DMLP. Choosing better hyperparameter values/variables for the network result in better performance. So, finding the best hyperparameters for the network is a significant issue. In literature, there are different methods to find best hyperparameters: Manual Search (MS), Grid Search (GS), RandomSearch (RS), Bayesian Methods (Sequential Model-Based Global Optimization (SMBGO), The Gaussian Process Approach (GPA), Tree-structured Parzen Estimator Approach (TSPEA)) [55, 56].

\subsection{Recurrent Neural Network (RNN)}

RNN is another type of DL network that is used for time series or sequential data, such as language and speech. RNNs are also used in traditional ML models (Back Propagation Through Time (BPTT), Jordan-Elman networks, etc.), however, the time lengths in such models are generally less than the models used in deep RNN models. Deep RNNs are preferred due to their ability to include longer time periods. Unlike Fully Connected Neural Networks (FNNs), RNNs use internal memory to process incoming inputs. RNNs are used in the analysis of time series data in various fields (handwriting recognition, speech recognition, etc. As stated in the literature, RNNs are good at predicting the next character in the text, language translation applications, sequential data processing $[45,37]$.

RNN model architecture consists of different number of layers and different type of units in each layer. The main difference between RNN and FNN is that each RNN unit takes the current and previous input data at the same time. The output depends on the previous data in RNN model. The RNNs process input sequences one by one at any given time, during 
their operation. In the units on the hidden layer, they hold information about the history of the input in the "state vector". When the output of the units in the hidden layer is divided into different discrete time steps, the RNNs are converted into a DMLP [37]. In Figure 2, the information flow in the RNN's hidden layer is divided into discrete times. The status of the node $S$ at different times of $t$ is shown as $s_{t}$, the input value $x$ at different times is $x_{t}$, and the output value $o$ at different times is shown as $o_{t}$. Parameter values $(U, W, V)$ are always used in the same step.

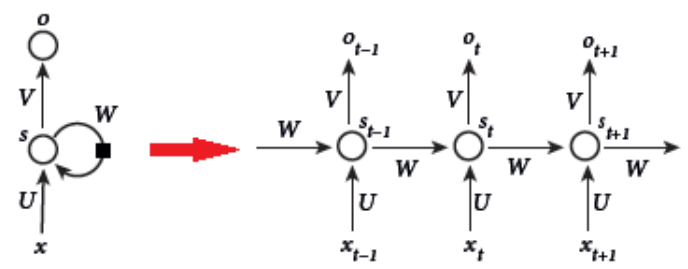

Figure 2: RNN cell through time[37]

RNNs can be trained using the BPTT algorithm. Optimization algorithms (SGD, RMSProp, ADAM) are used for weight adjustment process. With the BPTT learning method, the error change at any $t$ time is reflected in the input and weights of the previous $t$ times. The difficulty of training RNN is due to the fact that the RNN structure has a backward dependence over time. Therefore, RNNs become very complex in terms of the learning period. Although the main aim of using $\mathrm{RNN}$ is to learn long-term dependencies, studies in the literature show that when knowledge is stored for long time periods, it is not easy to learn with RNN (training difficulties on RNN) [57]. In order to solve this particular problem, LSTMs with different structures of ANN were developed [37]. Equations 8, 9 illustrate simpler RNN formulations. Equation 10 shows the total error which is the sum of each error at time step $t^{1}$.

$$
\begin{gathered}
h_{t}=W f\left(h_{t-1}\right)+W^{(h x)} x_{[t]} \\
y_{t}=W^{(S)} f\left(h_{t}\right) \\
\frac{\partial E}{\partial W}=\sum_{t=1}^{T} \frac{\partial E_{t}}{\partial W}
\end{gathered}
$$

Hyperparameters of RNN also define the network architecture and the performance of the network is affected by the parameter choices as was in DMLP case. The number of hidden layers, the number of units in each layer, regularization techniques, network weight initialization, activation functions, learning rate, momentum values, number of epochs, batch

\footnotetext{
${ }^{1}$ Richard Socher, CS224d: Deep Learning for Natural Language Processing, Lecture Notes
} 
size (minibatch size), decay rate, optimization algorithms, model of RNN (Vanilla RNN, Gated-Recurrent Unit (GRU), LSTM), sequence length for RNN are the hyperparameters of RNN. Finding the best hyperparameters for the network is a significant issue. In literature, there are different methods to find best hyperparameters: MS, GS, RS, Bayesian Methods (SMBGO, GPA, TSPEA) [55, 56].

\subsection{Long Short Term Memory (LSTM)}

LSTM [58] is a type of RNN where the network can remember both short term and long term values. LSTM networks are the preferred choice of many DL model developers when tackling complex problems like automatic speech recognition, and handwritten character recognition. LSTM models are mostly used with time-series data. It is used in different applications such as Natural Language Processing (NLP), language modeling, language translation, speech recognition, sentiment analysis, predictive analysis, financial time series analysis, etc. [59, 60]. With attention modules and AE structures, LSTM networks can be more successful on time series data analysis such as language translation [59].

LSTM networks consist of LSTM units. Each LSTM unit merges to form an LSTM layer. An LSTM unit is composed of cells having input gate, output gate and forget gate. Three gates regulate the information flow. With these features, each cell remembers the desired values over arbitrary time intervals. Equations 11-15 show the form of the forward pass of the LSTM unit [58] ( $x_{t}$ : input vector to the LSTM unit, $f_{t}$ : forget gate's activation vector, $i_{t}$ : input gate's activation vector, $o_{t}$ : output gate's activation vector, $h_{t}$ : output vector of the LSTM unit, $c_{t}$ : cell state vector, $\sigma_{g}$ : sigmoid function, $\sigma_{c}, \sigma_{h}$ : hyperbolic tangent function, *: element-wise (Hadamard) product, $W, U$ : weight matrices that need to be learned, $b$ : bias vector parameters that need to be learned) [60].

$$
\begin{gathered}
f_{t}=\sigma_{g}\left(W_{f} x_{t}+U_{f} h_{t-1}+b_{f}\right) \\
i_{t}=\sigma_{g}\left(W_{i} x_{t}+U_{i} h_{t-1}+b_{i}\right) \\
o_{t}=\sigma_{g}\left(W_{o} x_{t}+U_{o} h_{t-1}+b_{o}\right) \\
c_{t}=f_{t} * c_{t-1}+i_{t} * \sigma_{c}\left(W_{c} x_{t}+U_{c} h_{t-1}+b_{c}\right) \\
h_{t}=o_{t} * \sigma_{h}\left(c_{t}\right)
\end{gathered}
$$

LSTM is a specialized version of RNN. Therefore, the weight updates and preferred optimization methods are the same. In addition, the hyperparameters of LSTM are just like RNN: the number of hidden layers, the number of units in each layer, network weight initialization, activation functions, learning rate, momentum values, the number of epochs, batch size (minibatch size), decay rate, optimization algorithms, sequence length for LSTM, gradient clipping, gradient normalization, and dropout[61, 60]. In order to find the best hyperparameters of LSTM, the hyperparameter optimization methods that are used for RNN are also applicable to $\operatorname{LSTM}[55,56]$. 


\subsection{Convolutional Neural Networks (CNNs)}

CNN is a type of DNN that consists of convolutional layers that are based on the convolutional operation. Meanwhile, $\mathrm{CNN}$ is the most common model that is frequently used for vision or image processing based classification problems (image classification, object detection, image segmentation, etc.) $[62,63,64]$. The advantage of the usage of CNN is the number of parameters when comparing the vanilla DL models such as DMLP. Filtering with kernel window function gives an advantage of image processing to CNN architectures with fewer parameters that are beneficial for computing and storage. In CNN architectures, there are different layers: convolutional, max-pooling, dropout and fully connected Multilayer Perceptron (MLP) layer. The convolutional layer consists of the convolution (filtering) operation. Basic convolution operation is shown in Equation 16 ( $t$ denotes time, $s$ denotes feature map, $w$ denotes kernel, $x$ denotes input, $a$ denotes variable). In addition, the convolution operation is implemented on two-dimensional images. Equation 17 shows the convolution operation of two-dimensional image ( $I$ denotes input image, $K$ denotes the kernel, $m$ and $n$ denote the dimension of images, $i$ and $j$ denote variables). Besides, consecutive convolutional and max-pooling layers construct the deep network. Equation 18 provides the details about the NN architecture ( $W$ denotes weights, $x$ denotes input, $b$ denotes bias, $z$ denotes the output of neurons). At the end of the network, the softmax function is used to get the output. Equation 19 and 20 illustrate the softmax function ( $y$ denotes output) [39].

$$
\begin{gathered}
s(t)=(x * w)(t)=\sum_{a=-\infty}^{\infty} x(a) w(t-a) \\
S(i, j)=(I * K)(i, j)=\sum_{m} \sum_{n} I(m, n) K(i-m, j-n) . \\
z_{i}=\sum_{j} W_{i},_{j} x_{j}+b_{i} . \\
y=\operatorname{softmax}(z) \\
\operatorname{softmax}\left(z_{i}\right)=\frac{\exp \left(z_{i}\right)}{\sum_{j} \exp \left(z_{j}\right)}
\end{gathered}
$$

The backpropagation process is used for model learning of CNN. Most commonly used optimization algorithms (SGD, RMSProp) are used to find optimum parameters of CNN. Hyperparameters of CNN are similar to other DL model hyperparameters: the number of hidden layers, the number of units in each layer, network weight initialization, activation functions, learning rate, momentum values, the number of epochs, batch size (minibatch size), decay rate, optimization algorithms, dropout, kernel size, and filter size. In order to find the best hyperparameters of CNN, usual search algorithms are used: MS, GS, RS, and Bayesian Methods. [55, 56]. 


\subsection{Restricted Boltzmann Machines (RBMs)}

RBM is a productive stochastic ANN that can learn probability distribution on the input set [65]. RBMs are mostly used for unsupervised learning [66]. RBMs are used in applications such as dimension reduction, classification, feature learning, collaborative filtering [67]. The advantage of the RBMs is to find hidden patterns with an unsupervised method. The disadvantage of RBMs is its difficult training process. "RBMs are tricky because although there are good estimators of the log-likelihood gradient, there are no known cheap ways of estimating the log-likelihood itself" [68].

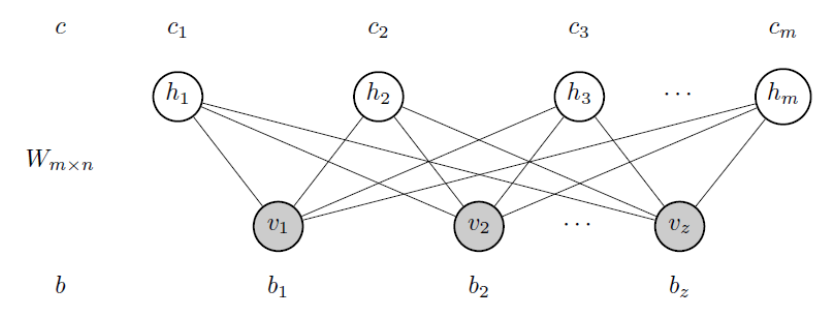

Figure 3: RBM Visible and Hidden Layers [65]

RBM is a two-layer, bipartite, and undirected graphical model that consists of two layers; visible and hidden layers (Figure 3). The layers are not connected among themselves. Each cell is a computational point that processes the input and makes stochastic decisions about whether this nerve node will transmit the input. Inputs are multiplied by specific weights, certain threshold values (bias) are added to input values, then calculated values are passed through an activation function. In reconstruction stage, the results in the outputs re-enter the network as the input, then they exit from the visible layer as the output. The values of the previous input and the values after the processes are compared. The purpose of the comparison is to reduce the difference.

Equation 21 illustrates the probabilistic semantics for an RBM by using its energy function $(P$ denotes the probabilistic semantics for an RBM, $Z$ denotes the partition function, $E$ denotes the energy function, $h$ denotes hidden units, $v$ denotes visible units).Equation 22 illustrates the partition function or the normalizing constant. Equation 23 shows the energy of a configuration (in matrix notation) of the standard type of RBM that has binary-valued hidden and visible units ( $a$ denotes bias weights (offsets) for the visible units, $b$ denotes bias weights for the hidden units, $W$ denotes matrix weight of the connection between hidden and visible units, $T$ denotes the transpose of matrix, $v$ denotes visible units, $h$ denotes hidden units) [69, 70].

$$
\begin{aligned}
& P(v, h)=\frac{1}{Z} \exp (-E(v, h)) \\
& Z=\sum_{v} \sum_{h} \exp (-E(v, h))
\end{aligned}
$$




$$
E(v, h)=-a^{T} v-b^{T} h-v^{T} W h
$$

The learning is performed multiple times on the network [65]. The training of RBMs is implemented through minimizing the negative log-likelihood of the model and data. Contrastive Divergence (CD) algorithm is used for the stochastic approximation algorithm which replaces the model expectation for an estimation using Gibbs Sampling with a limited number of iterations [66]. In the CD algorithm, the Kullback Leibler Divergence (KL-Divergence) algorithm is used to measure the distance between its reconstructed probability distribution and the original probability distribution of the input [71].

Momentum, learning rate, weight-cost (decay rate), batch size (minibatch size), regularization method, the number of epochs, the number of layers, initialization of weights, size of visible units, size of hidden units, type of activation units (sigmoid, softmax, ReLU, Gaussian units, etc.), loss function, and optimization algorithms are the hyperparameters of RBMs. Similar to the other deep networks, the hyperparameters are searched with MS, GS, $\mathrm{RS}$, and bayesian methods (Gaussian process). In addition to these, Annealed Importance Sampling (AIS) is used to estimate the partition function. CD algorithm is also used for the optimization of RBMs [55, 56, 72, 73].

\subsection{Deep Belief Networks (DBNs)}

DBN is a type of deep ANN and consists of a stack of RBM networks (Figure 4). DBN is a probabilistic generative model that consists of latent variables. In DBN, there is no link between units in each layer. DBNs are used to find discriminate independent features in the input set using unsupervised learning [69]. The ability to encode the higher-order network structures and fast inference are the advantages of the DBNs [74]. DBNs have disadvantages of training like RBMs which is mentioned in the RBM section, (DBNs are composed of RBMs).



Figure 4: Deep Belief Network [65]

When DBN is trained on the training set in an unsupervised manner, it can learn to reconstruct the input set in a probabilistic way. Then the layers on the network begin to detect discriminating features in the input. After this learning step, supervised learning 
is carried out to perform the classification [75]. Equation 24 illustrates the probability of generating a visible vector ( $W$ : matrix weight of connection between hidden unit $h$ and visible unit $v, p(h \mid W)$ : the prior distribution over hidden vectors) [69].

$$
p(v)=\sum_{h} p(h \mid W) p(v \mid h, W)
$$

DBN training process can be divided into two steps: stacked RBM learning and backpropagation learning. In stacked RBM learning, iterative CD algorithm is used [66]. In backpropagation learning, optimization algorithms (SGD, RMSProp, ADAM) are used to train network [74]. DBNs' hyperparameters are similar to RBMs' hyperparameters. Momentum, learning rate, weight-cost (decay rate), regularization method, batch size (minibatch size), the number of epochs, the number of layers, initialization of weights, the number of RBM stacks, size of visible units in RBMs' layers, size of hidden units in RBMs' layer, type of units (sigmoid, softmax, rectified, Gaussian units, etc.), network weight initialization, and optimization algorithms are the hyperparameters of DBNs. Similar to the other deep networks, the hyperparameters are searched with MS, GS, RS, and Bayesian methods. CD algorithm is also used for the optimization of DBNs [55, 56, 72, 73].

\subsection{Autoencoders (AEs)}

AE networks are ANN types that are used as unsupervised learning models. In addition, AE networks are commonly used in DL models, wherein they remap the inputs (features) such that the inputs are more representative for classification. In other words, AE networks perform an unsupervised feature learning process, which fits very well with the DL theme. A representation of a data set is learned by reducing the dimensionality with AEs. AEs are similar to Feedforward Neural Networks (FFNNs)' architecture. They consist of an input layer, an output layer and one or more hidden layers that connect them together. The number of nodes in the input layer and the number of nodes in the output layer are equal to each other in AEs, and they have a symmetrical structure. The most notable advantages of AEs are dimensionality reduction and feature learning. Meanwhile, reducing dimensionality and feature extraction in AEs cause some drawbacks. Focusing on minimizing the loss of data relationship in encoding of $\mathrm{AE}$ cause the loss of some significant data relationships. Hence, this may be considered as a drawback of AEs[76].

In general, AEs contain two components: encoder and decoder. The input $x \in[0,1]^{d}$ is converted through function $f(x)$ ( $W_{1}$ denotes a weight matrix of encoder, $b_{1}$ denotes a bias vector of encoder, $\sigma_{1}$ element-wise sigmoid activation function of encoder). Output $h$ is the encoded part of AEs (code), latent variables, or latent representation. The inverse of function $f(x)$, called function $g(h)$, produces the reconstruction of output $r\left(W_{2}\right.$ denotes a weight matrix of decoder, $b_{2}$ denotes a bias vector of decoder, $\sigma_{2}$ element-wise sigmoid activation function of decoder). Equations 25 and 26 illustrate the simple AE process [77]. Equation 27 shows the loss function of the AE, the Mean Squared Error (MSE). In the literature, AEs have been used for feature extraction and dimensionality reduction [39, 77].

$$
h=f(x)=\sigma_{1}\left(W_{1} x+b_{1}\right)
$$




$$
\begin{gathered}
r=g(h)=\sigma_{2}\left(W_{2} h+b_{2}\right) \\
L(x, r)=\|x-r\|^{2}
\end{gathered}
$$

AEs are a specialized version of FFNNs. The backpropagation learning is used for the update of the weights in the network[39]. Optimization algorithms (SGD, RMSProp, ADAM) are used for the learning process of AEs. MSE is used as a loss function in AEs. In addition, recirculation algorithms may also be used for the training of the AEs [39]. AEs' hyperparameters are similar to DL hyperparameters. Learning rate, weight-cost (decay rate), dropout fraction, batch size (minibatch size), the number of epochs, the number of layers, the number of nodes in each encoder layers, type of activation functions, number of nodes in each decoder layers, network weight initialization, optimization algorithms, and the number of nodes in the code layer (size of latent representation) are the hyperparameters of AEs. Similar to the other deep networks, the hyperparameters are searched with MS, GS, RS, and Bayesian methods [55, 56].

\subsection{Deep Reinforcement Learning (DRL)}

Reinforcement learning (RL) is a type of learning method that differs from supervised and unsupervised learning models. It does not need a preliminary data set which is labeled or clustered before. RL is an ML approach inspired by learning action/behavior, which deals with what actions should be taken by subjects to achieve the highest reward in an environment. There are different application areas that are used: game theory, control theory, multi-agent systems, operations research, robotics, information theory, managing investment portfolio, simulation-based optimization, playing Atari games, and statistics [78]. Some of the advantages of using RL for control problems are that an agent can be easily re-trained to adapt to changes in the environment and that the system is continually improved while training is constantly performed. An RL agent learns by interacting with its surroundings and observing the results of these interactions. This learning method mimics the basic way of how people learn.

RL is mainly based on Markov Decision Process (MDP). MDP is used to formalize the RL environment. MDP consists of five tuples: state (finite set of states), action (finite set of actions), reward function (scalar feedback signal), state transition probability matrix $\left(p\left(s^{\prime}, r \mid s, a\right), s^{\prime}\right.$ denotes next state, $r$ denotes reward function, $s$ denotes state, a denotes action), discount factor ( $\gamma$, present value of future rewards). The aim of the agent is to maximize the cumulative reward. The return $\left(G_{t}\right)$ is the total discounted reward. Equation 28 illustrates the total return $\left(G_{t}\right.$ denotes total discounted reward, $R$ denotes rewards, $t$ denotes time, $k$ denotes variable in time).

$$
G_{t}=R_{t+1}+\gamma R_{t+2}+\gamma^{2} R_{t+3}+\ldots=\sum_{k=0}^{\infty} \gamma^{k} R_{t+k+1}
$$

The value function is the prediction of the future values. It informs about how good is state/action. Equation 29 illustrates the formulation of the value function $(v(s)$ denotes 
the value function, $E[$.$] denotes the expectation function, G_{t}$ denotes the total discounted reward, $s$ denotes the given state, $R$ denotes the rewards, $S$ denotes the set of states, $t$ denotes time).

$$
v(s)=E\left[G_{t} \mid S_{t}=s\right]=E\left[R_{t+1}+\gamma v\left(S_{t+1}\right) \mid S_{t}=s\right]
$$

Policy $(\pi)$ is the agent's behavior strategy. It is like a map from state to action. There are two types of value functions to express the actions in the policy: state-value function $\left(v_{\pi}(s)\right)$, action-value function $\left(q_{\pi}(s, a)\right)$. The state-value function (Equation 30) is the expected return of starting from $s$ to following policy $\pi$ ( $E_{\pi}[$.$] denotes expectation function). The$ action-value function (Equation 31) is the expected return of starting from $s$, taking action a to following policy $\pi$ ( $A$ denotes the set of actions, $a$ denotes the given action).

$$
\begin{gathered}
v_{\pi}(s)=E_{\pi}\left[G_{t} \mid S_{t}=s\right]=E_{\pi}\left[\sum_{k=0}^{\infty} \gamma^{k} R_{t+k+1} \mid S_{t}=s\right] \\
q_{\pi}(s, a)=E_{\pi}\left[G_{t} \mid S_{t}=s, A_{t}=a\right]
\end{gathered}
$$

The optimal state-value function (Equation 32) is the maximum value function over all policies. The optimal action-value function (Equation 33) is the maximum action-value function over all policies.

$$
\begin{gathered}
v_{*}(s)=\max \left(v_{\pi}(s)\right) \\
q_{*}(s, a)=\max \left(q_{\pi}(s, a)\right)
\end{gathered}
$$

The RL solutions and methods in the literature are too broad to review in this paper. So, we summarized the important issues of RL, important RL solutions and methods. RL methods are mainly divided into two sections: Model-based methods and model-free methods. The model-based method uses a model that is known by the agent before, value/policy and experience. The experience can be real (sample from the environment) or simulated (sample from the model). Model-based methods are mostly used in the application of robotics, and control algorithms [79]. Model-free methods are mainly divided into two groups: Value-based and policy-based methods. In value-based methods, a policy is produced directly from the value function (e.g. epsilon-greedy). In policy-based methods, the policy is parametrized directly. In value-based methods, there are three main solutions for MDP problems: Dynamic Programming (DP), Monte Carlo (MC), and Temporal Difference (TD).

In DP method, problems are solved with optimal substructure and overlapping subproblems. The full model is known and it is used for planning in MDP. There are two iterations (learning algorithms) in DP: policy iteration and value iteration. MC method learns experience directly by running an episode of game/simulation. MC is a type of model-free method that does not need MDP transitions/rewards. It collects states, returns and it gets mean of returns for the value function. TD is also a model-free method that learns the experience directly by running the episode. In addition, TD learns incomplete episodes like the 
DP method by using bootstrapping. TD method combines MC and DP methods. SARSA (state, action, reward, state, action; $S_{t}, A_{t}, R_{t}, S_{t+1}, A_{t+1}$ ) is a type of TD control algorithm. Q-value (action-value function) is updated with the agent actions. It is an on-policy learning model that learns from actions according to the current policy $\pi$. Equation 34 illustrates the update of the action-value function in SARSA algorithm $\left(S_{t}\right.$ denotes current state, $A_{t}$ denotes current action, $t$ denotes time, $R$ denotes reward, $\alpha$ denotes learning rate, $\gamma$ denotes discount factor). Q-learning is another TD control algorithm. It is an off-policy learning model that learns from different actions that do not need the policy $\pi$ at all. Equation 35 illustrates the update of the action-value function in Q-Learning algorithm (The whole algorithms can be reached in [78], $a^{\prime}$ denotes action).

$$
\begin{gathered}
Q\left(S_{t}, A_{t}\right)=Q\left(S_{t}, A_{t}\right)+\alpha\left[R(t+1)+\gamma Q\left(S_{t+1}, A_{t+1}\right)-Q\left(S_{t}, A_{t}\right)\right] \\
Q\left(S_{t}, A_{t}\right)=Q\left(S_{t}, A_{t}\right)+\alpha\left[R(t+1)+\gamma \max _{a^{\prime}} Q\left(S_{t+1}, a^{\prime}\right)-Q\left(S_{t}, A_{t}\right)\right]
\end{gathered}
$$

In the value-based methods, a policy can be generated directly from the value function (e.g. using epsilon-greedy). The policy-based method uses the policy directly instead of using the value function. It has advantages and disadvantages over the value-based methods. The policy-based methods are more effective in high-dimensional or continuous action spaces, and have better convergence properties when compared against the value-based methods. It can also learn the stochastic policies. On the other hand, the policy-based method evaluates a policy that is typically inefficient and has high variance. It typically converges to a local rather than the global optimum. In the policy-based methods, there are also different solutions: Policy gradient, Reinforce (Monte-Carlo Policy Gradient), Actor-Critic [78] (Details of policy-based methods can be reached in [78]).

DRL methods contain NNs. Therefore, DRL hyperparameters are similar to DL hyperparameters. Learning rate, weight-cost (decay rate), dropout fraction, regularization method, batch size (minibatch size), the number of epochs, the number of layers, the number of nodes in each layer, type of activation functions, network weight initialization, optimization algorithms, discount factor, and the number of episodes are the hyperparameters of DRL. Similar to the other deep networks, the hyperparameters are searched with MS, GS, RS and bayesian methods $[55,56]$.

\section{Financial Time Series Forecasting}

The most widely studied financial application area is forecasting of a given financial time series, in particular asset price forecasting. Even though some variations exist, the main focus is on predicting the next movement of the underlying asset. More than half of the existing implementations of DL were focused on this area. Even though there are several subtopics of this general problem including Stock price forecasting, Index prediction, forex price prediction, commodity (oil, gold, etc) price prediction, bond price forecasting, volatility forecasting, cryptocurrency price forecasting, the underlying dynamics are the same in all of these applications. 
The studies can also be clustered into two main groups based on their expected outputs: price prediction and price movement (trend) prediction. Even though price forecasting is basically a regression problem, in most of the financial time series forecasting applications, correct prediction of the price is not perceived as important as correctly identifying the directional movement. As a result, researchers consider trend prediction, i.e. forecasting which way the price will change, a more crucial study area compared with exact price prediction. In that sense, trend prediction becomes a classification problem. In some studies, only up or down movements are taken into consideration (2-class problem), whereas up, down or neutral movements (3-class problem) also exist.

LSTM and its variations along with some hybrid models dominate the financial time series forecasting domain. LSTM, by its nature utilizes the temporal characteristics of any time series signal, hence forecasting financial time series is a well-studied and successful implementation of LSTM. However, some researchers prefer to either extract appropriate features from the time series or transform the time series in such a way that, the resulting financial data becomes stationary from a temporal perspective, meaning even if we shuffle the data order, we will still be able to properly train the model and achieve successful outof-sample test performance. For those implementations, CNN and Deep Feedforward Neural Network (DFNN) were the most commonly chosen DL models.

Various financial time series forecasting implementations using DL models exist in literature. We will cover each of these aforementioned implementation areas in the following subsections. In this survey paper, we examined the papers using the following criteria:

- First, we grouped the articles according to their subjects.

- Then, we grouped the related papers according to their feature set.

- Finally, we grouped each subgroup according to DL models/methods.

For each implementation area, the related papers will be subgrouped and tabulated. Each table will have the following fields to provide the information about the implementation details for the papers within the group: Article (Art.) and Data Set are trivial, Period refers to the time period for training and testing. Feature Set lists the input features used in the study. Lag has the time length of the input vector (e.g. 30d means the input vector has a 30 day window) and horizon shows how far out into the future is predicted by the model. Some abbreviations are used for these two aforementioned fields: min is minutes, $\mathrm{h}$ is hours, $\mathrm{d}$ is days, w is weeks, $\mathrm{m}$ is months, $\mathrm{y}$ is years, $\mathrm{s}$ is steps, ${ }^{*}$ is mixed. Method shows the DL models that are used in the study. Performance criteria provides the evaluation metrics, and finally the Environment (Env.) lists the development framework/software/tools. Some column values might be empty, indicating there was no relevant information in the paper for the corresponding field.

\subsection{Stock Price Forecasting}

Price prediction of any given stock is the most studied financial application of all. We observed the same trend within the DL implementations. Depending on the prediction time 
horizon, different input parameters are chosen varying from High Frequency Trading (HFT) and intraday price movements to daily, weekly or even monthly stock close prices. Also, technical, fundamental analysis, social media feeds, sentiment, etc. are among the different parameters that are used for the prediction models.

Table 1: Stock Price Forecasting Using Only Raw Time Series Data

\begin{tabular}{|c|c|c|c|c|c|c|c|c|}
\hline Art. & Data Set & Period & Feature Set & Lag & Horizon & Method & $\begin{array}{l}\text { Performance } \\
\text { Criteria }\end{array}$ & Env. \\
\hline [80] & 38 stocks in KOSPI & $2010-2014$ & $\begin{array}{l}\text { Lagged stock re- } \\
\text { turns }\end{array}$ & $50 \mathrm{~min}$ & $5 \mathrm{~min}$ & $\mathrm{DNN}$ & $\begin{array}{l}\text { NMSE, RMSE, } \\
\text { MAE, MI }\end{array}$ & - \\
\hline [81] & $\begin{array}{l}\text { China stock market, } \\
3049 \text { Stocks }\end{array}$ & $1990-2015$ & OCHLV & $30 \mathrm{~d}$ & $3 \mathrm{~d}$ & LSTM & Accuracy & $\begin{array}{l}\text { Theano, } \\
\text { Keras }\end{array}$ \\
\hline [82] & $\begin{array}{lcc}\text { Daily } & \text { returns } & \text { of } \\
\text { 'BRD' } & \text { stock } & \text { in }\end{array}$ & 2001-2016 & OCHLV & - & $1 \mathrm{~d}$ & LSTM & RMSE, MAE & $\begin{array}{l}\text { Python, } \\
\text { Theano }\end{array}$ \\
\hline & Romanian Market & & & & & & & \\
\hline [83] & $\begin{array}{l}297 \text { listed compa- } \\
\text { nies of CSE }\end{array}$ & $2012-2013$ & OCHLV & $2 \mathrm{~d}$ & $1 \mathrm{~d}$ & $\begin{array}{l}\text { LSTM, } \\
\text { SRNN, GRU }\end{array}$ & MAD, MAPE & Keras \\
\hline [84] & 5 stock in NSE & $1997-2016$ & 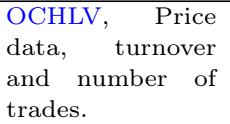 & $200 \mathrm{~d}$ & $1 . .10 \mathrm{~d}$ & $\begin{array}{l}\text { LSTM, RNN, } \\
\text { CNN, MLP }\end{array}$ & MAPE & - \\
\hline [85] & $\begin{array}{l}\text { Stocks of Infosys, } \\
\text { TCS and CIPLA } \\
\text { from NSE }\end{array}$ & 2014 & Price data & - & - & $\begin{array}{l}\text { RNN, LSTM } \\
\text { and CNN }\end{array}$ & Accuracy & - \\
\hline [86] & 10 stocks in $\mathrm{S} \& \mathrm{P} 500$ & $1997-2016$ & $\begin{array}{ll}\text { OCHLV, } & \text { Price } \\
\text { data } & \\
\end{array}$ & $36 \mathrm{~m}$ & $1 \mathrm{~m}$ & $\begin{array}{l}\text { RNN, LSTM, } \\
\text { GRU }\end{array}$ & $\begin{array}{l}\text { Accuracy, } \\
\text { Monthly return }\end{array}$ & $\begin{array}{l}\text { Keras, } \\
\text { Tensorflow }\end{array}$ \\
\hline [87] & $\begin{array}{l}\text { Stocks data from } \\
\text { S\&P500 }\end{array}$ & $2011-2016$ & OCHLV & $1 d$ & $1 d$ & $\mathrm{DBN}$ & $\begin{array}{l}\text { MSE, norm- } \\
\text { RMSE, MAE }\end{array}$ & - \\
\hline [88] & $\begin{array}{l}\text { High-frequency } \\
\text { transaction data of } \\
\text { the CSI300 futures }\end{array}$ & 2017 & Price data & - & $1 \mathrm{~min}$ & $\begin{array}{l}\text { DNN, ELM, } \\
\text { RBF }\end{array}$ & $\begin{array}{l}\text { RMSE, MAPE, } \\
\text { Accuracy }\end{array}$ & Matlab \\
\hline [89] & $\begin{array}{lll}\text { Stocks } & \text { in } & \text { the } \\
\text { S\&P500 } & & \end{array}$ & $1990-2015$ & Price data & $240 \mathrm{~d}$ & $1 d$ & $\begin{array}{l}\text { DNN, GBT, } \\
\text { RF }\end{array}$ & $\begin{array}{ll}\text { Mean } & \text { return, } \\
\text { MDD, } & \text { Calmar } \\
\text { ratio } & \\
\end{array}$ & $\mathrm{H} 2 \mathrm{O}$ \\
\hline [90] & $\begin{array}{l}\text { ACI Worldwide, } \\
\text { Staples, and Sea- } \\
\text { gate in NASDAQ }\end{array}$ & $2006-2010$ & $\begin{array}{l}\text { Daily } \quad \text { closing } \\
\text { prices }\end{array}$ & $17 \mathrm{~d}$ & $1 d$ & RNN, ANN & RMSE & - \\
\hline [91] & Chinese Stocks & $2007-2017$ & OCHLV & $30 \mathrm{~d}$ & $1 . .5 \mathrm{~d}$ & $\begin{array}{l}\text { CNN } \\
\text { LSTM }\end{array}$ & $\begin{array}{l}\text { Annualized } \\
\text { Return, Mxm } \\
\text { Retracement }\end{array}$ & Python \\
\hline [92] & 20 stocks in S\&P500 & $2010-2015$ & Price data & - & - & $\mathrm{AE}+\mathrm{LSTM}$ & Weekly Returns & - \\
\hline [93] & S\&P500 & $1985-2006$ & $\begin{array}{l}\text { Monthly and } \\
\text { daily log-returns }\end{array}$ & $*$ & $1 d$ & $\mathrm{DBN}+\mathrm{MLP}$ & $\begin{array}{l}\text { Validation, Test } \\
\text { Error }\end{array}$ & $\begin{array}{l}\text { Theano, } \\
\text { Python, } \\
\text { Matlab }\end{array}$ \\
\hline [94] & $\begin{array}{l}12 \text { stocks from SSE } \\
\text { Composite Index }\end{array}$ & $2000-2017$ & OCHLV & $60 \mathrm{~d}$ & $1 . .7 \mathrm{~d}$ & DWNN & MSE & Tensorflow \\
\hline [95] & $\begin{array}{ll}50 \quad \text { stocks } & \text { from } \\
\text { NYSE } & \\
\end{array}$ & $2007-2016$ & Price data & - & $\begin{array}{l}1 \mathrm{~d}, 3 \mathrm{~d}, \\
5 \mathrm{~d}\end{array}$ & SFM & MSE & - \\
\hline
\end{tabular}

In this survey, first, we grouped the stock price forecasting articles according to their feature set such as studies using only the raw time series data (price data, Open,Close,High, Low, Volume (OCHLV)) for price prediction; studies using various other data and papers that used text mining techniques. Regarding the first group, the corresponding DL models were directly implemented using the raw time series for price prediction. Table 1 tabulates the stock price forecasting papers that used only raw time series data in the literature. In Table 1, different methods/models are also listed based on four sub-groups: DNN (networks that are deep but without any given topology details) and LSTM models; multi models; hybrid models; novel methods. 
DNN and LSTM models were solely used in 3 papers. In [80], DNN and lagged stock returns were used to predict the stock prices in The Korea Composite Stock Price Index (KOSPI). Chen et. al. [81], Dezsi and Nistor [82] applied the raw price data as the input to LSTM models.

Meanwhile, there were some studies implementing multiple DL models for performance comparison using only the raw price (OCHLV) data for forecasting. Among the noteworthy studies, the authors in [83] compared RNN, Stacked Recurrent Neural Network (SRNN), LSTM and GRU. Hiransha et. al. [84] compared LSTM, RNN, CNN, MLP, whereas in [85] RNN, LSTM, CNN, Autoregressive Integrated Moving Average (ARIMA) were preferred, Lee and Yoo [86] compared 3 RNN models (SRNN, LSTM, GRU) for stock price prediction and then constructed a threshold based portfolio with selecting stocks according to the predictions and Li et. al. [87] implemented DBN. Finally, the authors of [88] compared 4 different ML models (1 DL model - AE and RBM), MLP, Radial Basis Function Neural Network (RBF) and Extreme Learning Machine (ELM) for predicting the next price in 1minute price data. They also compared the results with different sized datasets. The authors of [89] used price data and DNN, Gradient Boosted Trees (GBT), Random Forest (RF) methods for the prediction of the stocks in the Standard's \& Poor's 500 Index (S\&P500). In Chandra and Chan [90], co-operative neuro-evolution, RNN (Elman network) and DFNN were used for the prediction of stock prices in National Association of Securities Dealers Automated Quotations (NASDAQ) (ACI Worldwide, Staples, and Seagate).

Meanwhile, hybrid models were used in some of the papers. The author of [91] applied CNN+LSTM in their studies. Heaton et. al. [92] implemented smart indexing with AE. The authors of [93] combined DBN and MLP to construct a stock portfolio by predicting each stock's monthly log-return and choosing the only stocks that were expected to perform better than the performance of the median stock.

In addition, some novel approaches were adapted in some of the studies. The author of [94] proposed novel Deep and Wide Neural Network (DWNN) which is combination of RNN and CNN. The author of [95] implemented State Frequency Memory (SFM) recurrent network in their studies.

In another group of studies, some researchers again focused on LSTM based models. However, their input parameters came from various sources including the raw price data, technical and/or fundamental analysis, macroeconomic data, financial statements, news, investor sentiment, etc. Table 2 tabulates the stock price forecasting papers that used various data such as the raw price data, technical and/or fundamental analysis, macroeconomic data in the literature. In Table 2, different methods/models are also listed based on five subgroups: DNN model; LSTM and RNN models; multiple and hybrid models; CNN model; novel methods.

DNN models were used in some of the stock price forecasting papers within this group. In [96], DNN model and 25 fundamental features were used for the prediction of the Japan Index constituents. Feng et. al. [97] also used fundamental features and DNN model for the prediction. DNN model, macro economic data such as GDP, unemployment rate, inventories, etc. were used by the authors of [98] for the prediction of the U.S. low-level disaggregated macroeconomic time series. 
LSTM and RNN models were chosen in some of the studies. Kraus and Feuerriegel [99] implemented LSTM with transfer learning using text mining through financial news and the stock market data. Similarly, the author of [100] used LSTM to predict the stock's next day price using corporate action events and macro-economic index. Zhang and Tan [101] implemented DeepStockRanker, an LSTM based model for stock ranking using 11 technical indicators. In another study [102], the authors used the price time series and emotional data from text posts for predicting the stock opening price of the next day with LSTM network. Akita et. al. [103] used textual information and stock prices through Paragraph Vector + LSTM for forecasting the prices and the comparisons were provided with different classifiers. Ozbayoglu [104] used technical indicators along with the stock data on a JordanElman network for price prediction.

There were also multiple and hybrid models that used mostly technical analysis features as their inputs to the DL model. Several technical indicators were fed into LSTM and MLP networks in [105] for predicting intraday price prediction. Recently, Zhou et. al. [106] used GAN for minimizing Forecast error loss and Direction prediction loss (GAN-FD) model for stock price prediction and compared their model performances against ARIMA, ANN and Support Vector Machine (SVM). The authors of [107] used several technical indicator features and time series data with Principal Component Analysis (PCA) for dimensionality reduction cascaded with DNN (2-layer FFNN) for stock price prediction. In [108], the authors used Market microstructures based trade indicators as inputs into RNN with Graves LSTM detecting the buy-sell pressure of movements in Istanbul Stock Exchange Index (BIST) in order to perform the price prediction for intelligent stock trading. In [109], next month's return was predicted and top to be performed portfolios were constructed. Good monthly returns were achieved with LSTM and LSTM-MLP models.

Meanwhile, in some of the papers, CNN models were preferred. The authors of [110] used 250 features: order details, etc for the prediction of the private brokerage company's real data of risky transactions. They used CNN and LSTM for stock price forecasting. The authors of [111] used CNN model, fundamental, technical and market data for the prediction.

Novel methods were also developed in some of the studies. In [112], FI-2010 dataset: bid/ask and volume were used as the feature set for the forecast. In the study, they proposed Weighted Multichannel Time-series Regression (WMTR), Multilinear Discriminant Analysis (MDA). The authors of [113] used 57 characteristic features such as Market equity, Market Beta, Industry momentum, Asset growth, etc. as inputs to a Fama-French n-factor model DL for predicting monthly US equity returns in New York Stock Exchange (NYSE), American Stock Exchange (AMEX), or NASDAQ.

Table 2: Stock Price Forecasting Using Various Data

\begin{tabular}{|c|c|c|c|c|c|c|c|}
\hline Art. & Data Set & Period & Feature Set & Lag & Horizon Method & $\begin{array}{l}\text { Performance } \\
\text { Criteria }\end{array}$ & Env. \\
\hline [96] & $\begin{array}{ll}\text { Japan Index } & \text { con- } \\
\text { stituents } & \text { from } \\
\text { WorldScope } & \end{array}$ & $1990-2016$ & $\begin{array}{l}25 \text { Fundamental } \\
\text { Features }\end{array}$ & $10 \mathrm{~d}$ & $\mathrm{DNN}$ & $\begin{array}{l}\text { Correlation, Ac- } \\
\text { curacy, MSE }\end{array}$ & Tensorflow \\
\hline [97] & Return of S\&P500 & $1926-2016$ & $\begin{array}{l}\text { Fundamental } \\
\text { Features: }\end{array}$ & - & DNN & MSPE & Tensorflow \\
\hline
\end{tabular}


Table 2: Stock Price Forecasting Using Various Data

\begin{tabular}{|c|c|c|c|c|c|c|c|c|}
\hline Art. & Data Set & Period & Feature Set & Lag & Horizon & Method & $\begin{array}{l}\text { Performance } \\
\text { Criteria }\end{array}$ & Env. \\
\hline [98] & $\begin{array}{l}\text { U.S. low-level disag- } \\
\text { gregated macroeco- } \\
\text { nomic time series }\end{array}$ & 1959-2008 & $\begin{array}{l}\text { GDP, Unemploy- } \\
\text { ment rate, Inven- } \\
\text { tories, etc. }\end{array}$ & - & - & DNN & $\mathrm{R}^{2}$ & - \\
\hline [99] & $\begin{array}{l}\text { CDAX stock market } \\
\text { data }\end{array}$ & $2010-2013$ & $\begin{array}{lr}\text { Financial news, } \\
\text { stock market } \\
\text { data }\end{array}$ & $20 \mathrm{~d}$ & $1 d$ & LSTM & $\begin{array}{lr}\text { MSE, } & \text { RMSE, } \\
\text { MAE, Accuracy, } \\
\text { AUC }\end{array}$ & $\begin{array}{l}\text { TensorFlow, } \\
\text { Theano, } \\
\text { Python, } \\
\text { Scikit- } \\
\text { Learn }\end{array}$ \\
\hline [100] & $\begin{array}{l}\text { Stock of Tsugami } \\
\text { Corporation }\end{array}$ & 2013 & Price data & - & - & LSTM & RMSE & $\begin{array}{l}\text { Keras, } \\
\text { Tensorflow }\end{array}$ \\
\hline [101] & $\begin{array}{l}\text { Stocks in China's A- } \\
\text { share }\end{array}$ & 2006-2007 & $\begin{array}{l}11 \text { technical indi- } \\
\text { cators }\end{array}$ & - & $1 d$ & LSTM & $\mathrm{AR}, \mathrm{IR}, \mathrm{IC}$ & - \\
\hline$[102]$ & SCI prices & $2008-2015$ & $\begin{array}{l}\text { OCHL of change } \\
\text { rate, price }\end{array}$ & $7 \mathrm{~d}$ & - & $\begin{array}{l}\text { EmotionalAna } \\
+ \text { LSTM }\end{array}$ & SildSE & - \\
\hline [103] & $\begin{array}{l}10 \text { stocks in Nikkei } \\
225 \text { and news }\end{array}$ & $2001-2008$ & $\begin{array}{l}\text { Textual informa- } \\
\text { tion and Stock } \\
\text { prices }\end{array}$ & $10 \mathrm{~d}$ & - & $\begin{array}{l}\text { Paragraph } \\
\text { Vector } \\
\text { LSTM }\end{array}$ & Profit & - \\
\hline [104] & $\begin{array}{l}\text { TKC stock in NYSE } \\
\text { and QQQQ ETF }\end{array}$ & 1999-2006 & $\begin{array}{l}\text { Technical indica- } \\
\text { tors, Price }\end{array}$ & $50 \mathrm{~d}$ & $1 d$ & $\begin{array}{l}\text { RNN } \\
\text { (Jordan- } \\
\text { Elman) }\end{array}$ & Profit, MSE & Java \\
\hline [105] & 10 Stocks in NYSE & - & $\begin{array}{l}\text { Price data, Tech- } \\
\text { nical indicators }\end{array}$ & $20 \mathrm{~min}$ & $1 \mathrm{~min}$ & LSTM, MLP & RMSE & - \\
\hline [106] & $\begin{array}{l}42 \text { stocks in China's } \\
\text { SSE }\end{array}$ & 2016 & $\begin{array}{l}\text { OCHLV, Techni- } \\
\text { cal Indicators }\end{array}$ & $242 \mathrm{~min}$ & $1 \mathrm{~min}$ & $\begin{array}{l}\text { GAN } \\
(\mathrm{LSTM} \\
\mathrm{CNN})\end{array}$ & $\begin{array}{l}\text { RMSRE, DPA, } \\
\text { GAN-F, GAN-D }\end{array}$ & - \\
\hline [107] & $\begin{array}{l}\text { Google's daily stock } \\
\text { data }\end{array}$ & 2004-2015 & $\begin{array}{l}\text { OCHLV, Techni- } \\
\text { cal indicators }\end{array}$ & $20 \mathrm{~d}$ & $1 \mathrm{~d}$ & $\begin{array}{l}(2 D)^{2} \\
+\mathrm{DNN}\end{array}$ & $\begin{array}{l}\text { SMAPE, PCD, } \\
\text { MAPE, RMSE, } \\
\text { HR, TR, } \mathrm{R}^{2}\end{array}$ & R, Matlab \\
\hline [108] & $\begin{array}{ll}\text { GarantiBank } & \text { in } \\
\text { BIST, Turkey } & \end{array}$ & 2016 & $\begin{array}{l}\text { OCHLV, Volatil- } \\
\text { ity, etc. }\end{array}$ & - & - & $\begin{array}{l}\text { PLR, Graves } \\
\text { LSTM }\end{array}$ & $\begin{array}{l}\text { MSE, RMSE, } \\
\text { MAE, RSE, } R^{2}\end{array}$ & Spark \\
\hline [109] & $\begin{array}{l}\text { Stocks in NYSE, } \\
\text { AMEX, NASDAQ, } \\
\text { TAQ intraday trade }\end{array}$ & 1993-2017 & $\begin{array}{l}\text { Price, } 15 \text { firm } \\
\text { characteristics }\end{array}$ & $80 \mathrm{~d}$ & $1 d$ & LSTM+MLP & $\begin{array}{l}\text { Monthly return, } \\
\text { SR }\end{array}$ & $\begin{array}{l}\text { Python,Keras } \\
\text { Tensorflow } \\
\text { in AWS } \\
\end{array}$ \\
\hline [110] & $\begin{array}{l}\text { Private brokerage } \\
\text { company's real data } \\
\text { of risky transactions }\end{array}$ & - & $\begin{array}{l}250 \text { features: or- } \\
\text { der details, etc. }\end{array}$ & - & - & CNN, LSTM & F1-Score & $\begin{array}{l}\text { Keras, } \\
\text { Tensorflow }\end{array}$ \\
\hline [111] & $\begin{array}{l}\text { Fundamental and } \\
\text { Technical Data, } \\
\text { Economic Data }\end{array}$ & - & $\begin{array}{l}\text { Fundamental } \\
\text { technical and } \\
\text { market informa- } \\
\text { tion }\end{array}$ & - & - & $\mathrm{CNN}$ & - & - \\
\hline [112] & $\begin{array}{l}\text { The LOB of } 5 \text { stocks } \\
\text { of Finnish Stock } \\
\text { Market }\end{array}$ & 2010 & $\begin{array}{l}\text { FI-2010 dataset: } \\
\text { bid/ask and vol- } \\
\text { ume }\end{array}$ & - & $*$ & $\begin{array}{l}\text { WMTR, } \\
\text { MDA }\end{array}$ & $\begin{array}{l}\text { Accuracy, Preci- } \\
\text { sion, Recall, F1- } \\
\text { Score }\end{array}$ & - \\
\hline [113] & $\begin{array}{l}\text { Returns in NYSE, } \\
\text { AMEX, NASDAQ }\end{array}$ & $1975-2017$ & $\begin{array}{l}57 \text { firm character- } \\
\text { istics }\end{array}$ & $*$ & - & $\begin{array}{l}\text { Fama-French } \\
\text { n-factor } \\
\text { model DL }\end{array}$ & $\mathrm{R}^{2}, \mathrm{RMSE}$ & Tensorflow \\
\hline
\end{tabular}

There were a number of research papers that also used text mining techniques for the feature extraction, but used non-LSTM models for the stock price prediction. Table 3 tabulates the stock price forecasting papers that used text mining techniques. In Table 3, different methods/models are clustered into three sub-groups: CNN and LSTM models; GRU, LSTM, and RNN models; novel methods.

CNN and LSTM models were adapted in some of the papers. In [114], events were detected from Reuters and Bloomberg news through text mining and that information was used for the price prediction and stock trading through the CNN model. Vargas et. al. [115] used text mining on S\&P500 index news from Reuters through a LSTM+CNN hybrid model for price prediction and intraday directional movement estimation together. The authors of 
[116] used the financial news data and implemented word embedding with Word2vec along with MA and stochastic oscillator to create inputs for Recurrent CNN (RCNN) for stock price prediction. The authors of [117] also used sentiment analyses through text mining and word embeddings from analyst reports and used sentiment features as inputs to DFNN model for stock price prediction. Then different portfolio selections were implemented based on the projected stock returns.

GRU, LSTM, and RNN models were preferred in the next group of papers. Das et. al. [118] implemented sentiment analysis on Twitter posts along with the stock data for price forecasting using RNN. Similarly, the authors of [119] used sentiment classification (neutral, positive, negative) for the stock open or close price prediction with various LSTM models. They compared their results with SVM and achieved higher overall performance. In [120], text and price data were used for the prediction of the SSE Composite Index (SCI) prices.

Some novel approaches were also found in some of the papers. The authors of [121] used word embeddings for extracting information from web pages and then combined with the stock price data for stock price prediction. They compared Autoregressive (AR) model and $\mathrm{RF}$ with and without news. The results showed embedding news information improved the performance. In [122], financial news and ACE2005 Chinese corpus were used. Different event-types on Chinese companies were classified based on a novel event-type pattern classification algorithm in [122], also next day stock price change was predicted using additional inputs.

Table 3: Stock Price Forecasting Using Text Mining Techniques for Feature Extrac-

tion

\begin{tabular}{|c|c|c|c|c|c|c|c|c|}
\hline Art. & Data Set & Period & Feature Set & Lag & Horizon & Method & $\begin{array}{l}\text { Performance } \\
\text { Criteria }\end{array}$ & Env. \\
\hline [114] & $\begin{array}{l}\text { S\&P500 Index, } 15 \\
\text { stocks in S\&P500 }\end{array}$ & 2006-2013 & $\begin{array}{lr}\text { News } & \text { from } \\
\text { Reuters } & \text { and } \\
\text { Bloomberg } & \\
\end{array}$ & - & - & $\mathrm{CNN}$ & Accuracy, MCC & - \\
\hline [115] & $\begin{array}{l}\text { S\&P500 index news } \\
\text { from Reuters }\end{array}$ & $2006-2013$ & $\begin{array}{l}\text { Financial news } \\
\text { titles, Technical } \\
\text { indicators }\end{array}$ & $1 \mathrm{~d}$ & $1 \mathrm{~d}$ & RCNN & Accuracy & - \\
\hline [116] & $\begin{array}{lcc}\text { TWSE } & \text { index, } & 4 \\
\text { stocks in TWSE } & \end{array}$ & $2001-2017$ & $\begin{array}{l}\text { Technical indica- } \\
\text { tors, Price data, } \\
\text { News }\end{array}$ & $15 \mathrm{~d}$ & - & $\begin{array}{l}\text { CNN } \\
\text { LSTM }\end{array}$ & RMSE, Profit & $\begin{array}{l}\text { Keras, } \\
\text { Python, } \\
\text { TALIB }\end{array}$ \\
\hline$[117]$ & $\begin{array}{l}\text { Analyst reports on } \\
\text { the TSE and Osaka } \\
\text { Exchange }\end{array}$ & 2016-2018 & Text & - & - & $\begin{array}{l}\text { LSTM, CNN, } \\
\text { Bi-LSTM }\end{array}$ & $\begin{array}{l}\text { Accuracy, } \\
\text { squared }\end{array}$ & $\begin{array}{l}\text { R, } \\
\text { Python, } \\
\text { MeCab }\end{array}$ \\
\hline [118] & $\begin{array}{l}\text { Stocks of Google, } \\
\text { Microsoft and Apple }\end{array}$ & $2016-2017$ & $\begin{array}{ll}\text { Twitter } & \text { senti- } \\
\text { ment and } & \text { stock } \\
\text { prices } & \end{array}$ & - & - & RNN & - & $\begin{array}{l}\text { Spark, } \\
\text { Flume, } \\
\text { Twitter } \\
\text { API, }\end{array}$ \\
\hline [119] & $\begin{array}{l}\text { Stocks of CSI300 } \\
\text { index, OCHLV of } \\
\text { CSI300 index }\end{array}$ & 2009-2014 & $\begin{array}{l}\text { Sentiment Posts, } \\
\text { Price data }\end{array}$ & $1 \mathrm{~d}$ & $1 \mathrm{~d}$ & $\begin{array}{l}\text { Naive Bayes } \\
+ \text { LSTM }\end{array}$ & $\begin{array}{l}\text { Precision, Recall, } \\
\text { F1-score, Accu- } \\
\text { racy }\end{array}$ & $\begin{array}{l}\text { Python, } \\
\text { Keras }\end{array}$ \\
\hline [120] & SCI prices & $2013-2016$ & $\begin{array}{l}\text { Text data and } \\
\text { Price data }\end{array}$ & $7 \mathrm{~d}$ & $1 \mathrm{~d}$ & LSTM & $\begin{array}{l}\text { Accuracy, F1- } \\
\text { Measure }\end{array}$ & $\begin{array}{l}\text { Python, } \\
\text { Keras }\end{array}$ \\
\hline$[121]$ & Stocks from S\&P500 & 2006-2013 & $\begin{array}{l}\text { Text (news) and } \\
\text { Price data }\end{array}$ & $7 \mathrm{~d}$ & $1 \mathrm{~d}$ & $\begin{array}{l}\text { LAR+News, } \\
\mathrm{RF}+\text { News }\end{array}$ & MAPE, RMSE & - \\
\hline [122] & $\begin{array}{l}\text { News from } \\
\text { Sina.com, ACE2005 } \\
\text { Chinese corpus }\end{array}$ & $2012-2016$ & A set of news text & - & - & $\begin{array}{l}\text { Their unique } \\
\text { algorithm }\end{array}$ & $\begin{array}{l}\text { Precision, Recall, } \\
\text { F1-score }\end{array}$ & - \\
\hline
\end{tabular}




\subsection{Index Forecasting}

Instead of trying to forecast the price of a single stock, several researchers preferred to predict the stock market index. Indices generally are less volatile than individual stocks, since they are composed of multiple stocks from different sectors and are more indicative of the overall momentum and general state of the economy.

In the literature, different stock market index data were used for the experiments. Most commonly used index data can be listed as follows: S\&P500, China Securities Index (CSI)300, National Stock Exchange of India (NIFTY), Tokyo Nikkei Index (NIKKEI)225, Dow Jones Industrial Average (DJIA), Shanghai Stock Exchange (SSE)180, Hong Kong Hang Seng Index (HSI), Shenzhen Stock Exchange Composite Index (SZSE), London Financial Times Stock Exchange Index (FTSE)100, Taiwan Capitalization Weighted Stock Index (TAIEX), BIST, NASDAQ, Dow Jones Industrial Average 30 (DOW30), KOSPI, S\&P500 Volatility Index (VIX), NASDAQ100 Volatility Index (VXN), Brazilian Stock Exchange (Bovespa), Stockholm Stock Exchange (OMX), NYSE. The authors of [123, 124, 125, 126, 127, 128, 129, 130, 131, 132, 133, 134, 114] used S\&P500 as their dataset. The authors of $[123,124,135,136,137]$ used NIKKEI as their dataset. KOSPI was used in [135, 131, 132]. DJIA was used as the dataset in [123, 136, 137, 138, 139]. Besides, the authors of $[123,135,137,131]$ used HSI as the dataset in their studies. SZSE is used in studies of $[140,135,141,142]$.

In addition, in the literature, there were different methods for the prediction of the index data. While some of the studies used only the raw time series data, some others used various other data such as technical indicators, index data, social media feeds, news from Reuters, Bloomberg, the statistical features of data (standard deviation, skewness, kurtosis, omega ratio, fund alpha). In this survey, first, we grouped the index forecasting articles according to their feature set such as studies using only the raw time series data (price/index data, OCHLV); then in the second group we clustered the studies using various other data. Table 4 tabulates the index forecasting papers using only the raw time series data. Moreover, different methods (models) were used for index forecasting. MLP, RNN, LSTM, DNN (most probably DFNN, or DMLP) methods were the most used methods for index forecasting. In Table 4, these various methods/models are also listed as four sub-groups: ANN, DNN, MLP, and Fuzzy Deep Direct Reinforcement Learning (FDDR) models; RL and DL models; LSTM and RNN models; novel methods.

Table 4: Index Forecasting Using Only Raw Time Series Data

\begin{tabular}{|c|c|c|c|c|c|c|c|c|}
\hline Art. & Data Set & Period & Feature Set & Lag & Horizon & Method & $\begin{array}{l}\text { Performance } \\
\text { Criteria }\end{array}$ & Env. \\
\hline [124] & $\begin{array}{l}\text { S\&P500, Nikkei225, } \\
\text { USD Exchanges }\end{array}$ & 2011-2015 & Index data & - & $\begin{array}{l}1 \mathrm{~d}, \\
5 \mathrm{~d}, \\
7 \mathrm{~d}, \\
10 \mathrm{~d}\end{array}$ & $\begin{array}{l}\text { LRNFIS } \\
\text { with Firefly- } \\
\text { Harmony } \\
\text { Search }\end{array}$ & $\begin{array}{l}\text { RMSE, MAPE, } \\
\text { MAE }\end{array}$ & - \\
\hline$[125]$ & S\&P500 Index & 1989-2005 & $\begin{array}{l}\text { Index data, Vol- } \\
\text { ume }\end{array}$ & $240 \mathrm{~d}$ & $1 \mathrm{~d}$ & LSTM & $\begin{array}{l}\text { Return, STD, } \\
\text { SR, Accuracy }\end{array}$ & $\begin{array}{l}\text { Python, } \\
\text { Tensor- } \\
\text { Flow, } \\
\text { Keras, R, } \\
\text { H2O }\end{array}$ \\
\hline [127] & S\&P500, VIX & $2005-2016$ & Index data & * & $1 \mathrm{~d}$ & $\mathrm{uWN}, \mathrm{cWN}$ & $\begin{array}{l}\text { MASE, } \\
\text { RMSE }\end{array}$ & - \\
\hline
\end{tabular}


Table 4: Index Forecasting Using Only Raw Time Series Data

\begin{tabular}{|c|c|c|c|c|c|c|c|c|}
\hline Art. & Data Set & Period & Feature Set & Lag & Horizon & Method & $\begin{array}{l}\text { Performance } \\
\text { Criteria }\end{array}$ & Env. \\
\hline$[128]$ & S\&P500 Index & $2010-2017$ & Index data & $10 \mathrm{~d}$ & $\begin{array}{l}1 \mathrm{~d}, \\
30 \mathrm{~d}\end{array}$ & $\begin{array}{l}\text { Stacked } \\
\text { LSTM, Bi- } \\
\text { LSTM }\end{array}$ & $\begin{array}{l}\text { MAE, RMSE, R- } \\
\text { squared }\end{array}$ & $\begin{array}{l}\text { Python, } \\
\text { Keras, } \\
\text { Tensorflow }\end{array}$ \\
\hline [131] & $\begin{array}{l}\text { S\&P500, KOSPI, } \\
\text { HSI, and Eu- } \\
\text { roStoxx50 }\end{array}$ & $1987-2017$ & $\begin{array}{l}200 \text {-days stock } \\
\text { price }\end{array}$ & $200 \mathrm{~d}$ & $1 d$ & $\begin{array}{l}\text { Deep Q- } \\
\text { Learning and } \\
\text { DNN }\end{array}$ & $\begin{array}{l}\text { Total profit, Cor- } \\
\text { relation }\end{array}$ & - \\
\hline$[132]$ & $\begin{array}{l}\text { S\&P500, } \\
\text { KOSPI200, } \\
\text { stocks }\end{array}$ & $2000-2017$ & Index data & $20 \mathrm{~d}$ & $1 d$ & $\begin{array}{l}\text { ModAugNet: } \\
\text { LSTM }\end{array}$ & $\begin{array}{l}\text { MSE, } \\
\text { MAE }\end{array}$ & Keras \\
\hline [133] & $\begin{array}{l}\text { S\&P500, } \\
\text { Bovespa50, OMX30 }\end{array}$ & $2009-2017$ & $\begin{array}{l}\text { Autoregressive } \\
\text { part of the time } \\
\text { series }\end{array}$ & - & $1 d$ & LSTM & MSE, Accuracy & $\begin{array}{l}\text { Tensorflow, } \\
\text { Keras, R }\end{array}$ \\
\hline$[134]$ & S\&P500 & $2000-2017$ & Index data & - & $\begin{array}{l}1 . .4 \mathrm{~d}, \\
1 \mathrm{w} \\
1 . .3 \mathrm{~m} \\
\end{array}$ & $\begin{array}{l}\text { GLM, } \\
\text { LSTM+RNN }\end{array}$ & MAE, RMSE & Python \\
\hline$[136]$ & $\begin{array}{l}\text { Nikkei225, IXIC, } \\
\text { HSI, GSPC, DJIA }\end{array}$ & $1985-2018$ & OCHLV & $5 \mathrm{~d}$ & $1 d$ & LSTM & RMSE & $\begin{array}{l}\text { Python, } \\
\text { Keras, } \\
\text { Theano }\end{array}$ \\
\hline$[138]$ & DJIA & - & Index data & - & - & $\begin{array}{l}\text { Genetic Deep } \\
\text { Neural Net- } \\
\text { work }\end{array}$ & MSE & Java \\
\hline [139] & $\begin{array}{l}\text { Log returns of the } \\
\text { DJIA }\end{array}$ & $1971-2002$ & Index data & $20 \mathrm{~d}$ & $1 d$ & RNN & $\begin{array}{lr}\text { TR, sign } & \text { rate, } \\
\mathrm{PT} / \mathrm{HM} & \text { test, } \\
\mathrm{MSFE}, \mathrm{SR}, \text { profit }\end{array}$ & - \\
\hline$[140]$ & $\begin{array}{lr}\begin{array}{l}\text { Shanghai } \\
\text { composite }\end{array} & \text { index } \\
\text { SZSE } & \\
\end{array}$ & $2006-2016$ & OCHLV & $10 \mathrm{~d}$ & - & $\begin{array}{l}\text { Embedded } \\
\text { layer } \\
\text { LSTM }\end{array}$ & Accuracy, MSE & $\begin{array}{l}\text { Python, } \\
\text { Matlab, } \\
\text { Theano }\end{array}$ \\
\hline [141] & $\begin{array}{l}300 \text { stocks from } \\
\text { SZSE, Commodity }\end{array}$ & 2014-2015 & Index data & - & - & $\begin{array}{l}\text { FDDR, DNN } \\
+\mathrm{RL}\end{array}$ & $\begin{array}{l}\text { Profit, return, } \\
\text { SR, profit-loss } \\
\text { curves }\end{array}$ & Keras \\
\hline$[142]$ & $\begin{array}{l}\text { Shanghai composite } \\
\text { index and SZSE }\end{array}$ & $1990-2016$ & OCHLV & $20 \mathrm{~d}$ & $1 d$ & $\begin{array}{l}\text { Ensembles of } \\
\text { ANN }\end{array}$ & Accuracy & - \\
\hline$[143]$ & TUNINDEX & $2013-2017$ & $\begin{array}{l}\text { Log returns of in- } \\
\text { dex data }\end{array}$ & - & $5 \mathrm{~min}$ & $\begin{array}{l}\text { DNN with hi- } \\
\text { erarchical in- } \\
\text { put }\end{array}$ & Accuracy, MSE & Java \\
\hline$[144]$ & $\begin{array}{ll}\text { Singapore } & \text { Stock } \\
\text { Market Index } & \\
\end{array}$ & $2010-2017$ & $\begin{array}{l}\text { OCHL of last } 10 \\
\text { days of index }\end{array}$ & $10 \mathrm{~d}$ & $3 \mathrm{~d}$ & $\begin{array}{l}\text { Feed-forward } \\
\text { DNN }\end{array}$ & $\begin{array}{l}\text { RMSE, MAPE, } \\
\text { Profit, SR }\end{array}$ & - \\
\hline [145] & BIST & $1990-2002$ & Index data & $7 \mathrm{~d}$ & $1 d$ & $\begin{array}{l}\text { MLP, } \\
\text { MoE }\end{array}$ & $\begin{array}{l}\mathrm{HIT}, \text { posi- } \\
\text { tive/negative } \\
\text { HIT, MSE, MAE }\end{array}$ & - \\
\hline$[146]$ & SCI & $2012-2017$ & $\begin{array}{ll}\text { OCHLV, } & \text { Index } \\
\text { data } & \\
\end{array}$ & - & $1 . .10 \mathrm{~d}$ & $\begin{array}{l}\text { Wavelet } \quad+ \\
\text { LSTM }\end{array}$ & $\begin{array}{l}\text { MAPE, theil un- } \\
\text { equal coefficient }\end{array}$ & - \\
\hline$[147]$ & S\&P500 & $1950-2016$ & Index data & $15 \mathrm{~d}$ & $1 \mathrm{~d}$ & LSTM & RMSE & Keras \\
\hline [148] & ISE100 & $1987-2008$ & Index data & - & $\begin{array}{l}2 \mathrm{~d}, \\
4 \mathrm{~d}, \\
8 \mathrm{~d}, \\
12 \mathrm{~d}, \\
18 \mathrm{~d}\end{array}$ & $\begin{array}{l}\text { TAR-VEC- } \\
\text { MLP, TAR- } \\
\text { VEC-RBF, } \\
\text { TAR-VEC- } \\
\text { RHE } \\
\end{array}$ & RMSE & - \\
\hline [149] & VIX, VXN, VXD & $2002-2014$ & $\begin{array}{l}\text { First five autore- } \\
\text { gressive lags }\end{array}$ & $5 \mathrm{~d}$ & $\begin{array}{l}\mathrm{d}, \\
22 \mathrm{~d}\end{array}$ & $\begin{array}{l}\text { HAR- } \\
\text { GASVR }\end{array}$ & MAE, RMSE & - \\
\hline
\end{tabular}

ANN, DNN, MLP, and FDDR models were used in some of the studies. In [143], log returns of the index data was used with DNN with hierarchical input for the prediction of the TUNINDEX data. The authors of [144] used deep FFNN and Open,Close,High, Low (OCHL) of the last 10 days of index data for prediction. In addition, MLP and ANN were used for the prediction of index data. In [145], the raw index data was used with MLP, RNN, Mixture of Experts (MoE) and Exponential GARCH (EGARCH) for the forecast. In [142], ensembles of ANN with OCHLV of the data were used for the prediction of the 
Shanghai composite index.

Furthermore, RL and DL methods were used together for the prediction of the index data in some of the studies. In [141], FDDR, DNN and RL methods were used to predict 300 stocks from SZSE index data and commodity prices. In [131], Deep Q-Learning and DNN methods and 200-days stock price dataset were used together for the prediction of S\&P500 index.

Most of the preferred methods for prediction of the index data using the raw time series data were based on LSTM and RNN. In [139], RNN was used for prediction of the log returns of DJIA index. In [125], LSTM was used to predict S\&P500 Index data. The authors of [128] used stacked LSTM, Bidirectional LSTM (Bi-LSTM) methods for S\&P500 Index forecasting. The authors of [146] used LSTM network to predict the next day closing price of Shanghai stock Index. In their study, they used wavelet decomposition to reconstruct the financial time series for denoising and better learning. In [140], LSTM was used for the prediction of Shanghai A-shares composite index. The authors of [136] used LSTM to predict NIKKEI225, IXIC, HIS, GSPC and DJIA index data. In [147] and [132], LSTM was also used for the prediction of S\&P500 and KOSPI200 index. The authors of [132] developed an LSTM based stock index forecasting model called ModAugNet. The proposed method was able to beat Buy and Hold $(\mathrm{B} \& \mathrm{H})$ in the long term with an overfitting prevention mechanism. The authors of [134] compared different ML models (linear model), Generalized Linear Model (GML) and several LSTM, RNN models for stock index price prediction. In [133], LSTM and autoregressive part of the time series index data were used for prediction of S\&P500, Bovespa50, OMX30 indices.

Also, some studies adapted novel appraches. In [138], genetic DNN was used for DJIA index forecasting. The authors of [127] proposed a new DNN model which is called Wavenet convolutional net for time series forecasting. The authors of [148] proposed a (Threshold Autoregressive (TAR)-Vector Error Correction model (VEC)-Recurrent Hybrid Elman (RHE)) model for forex and stock index of return prediction and compared several models. The authors of [124] proposed a method that is called Locally Recurrent Neuro-fuzzy Information System (LRNFIS) with Firefly Harmony Search Optimization (FHSO) Evolutionary Algorithm (EA) to predict S\&P500, NIKKEI225 indices and USD Exchange price data. The authors of [149] proposed a Heterogeneous Autoregressive Process (HAR) with a GA with a SVR (GASVR) model that was called HAR-GASVR for prediction of VIX, VXN, Dow Jones Industrial Average Volatility Index (VXD) indices.

In the literature, some of the studies used various input data such as technical indicators, index data, social media news, news from Reuters, Bloomberg, the statistical features of data (standard deviation, skewness, kurtosis, omega ratio, fund alpha). Table 5 tabulates the index forecasting papers using these aforementioned various data. DNN, RNN, LSTM, CNN methods were the most commonly used models in index forecasting. In Table 5, different methods/models are also listed within four sub-groups: DNN model; RNN and LSTM models; CNN model; novel methods.

DNN was used as the classification model in some of the papers. In [150], DNN and some of the feature of the data (Return, Sharpe-ratio (SR), Standard Deviation (STD), Skewness, Kurtosis, Omega ratio, Fund alpha) were used for the prediction. In [126], DNN, RNN and 
technical indicators were used for the prediction of FTSE100, OMX30, S\&P500 indices.

In addition, RNN and LSTM models with various other data were also used for the prediction of the indices. The authors of [137] used RNN and OCHLV of indices, technical indicators to predict DJIA, FTSE, Nikkei, TAIEX indices. The authors of [151] used GASVR, LSTM for the forecast. The authors of [152] used four LSTM models (technical analysis, attention mechanism and market vector embedded) for the prediction of the daily return ratio of HSI300 index. In [135], LSTM with wavelet denoising and index data, volume, technical indicators were used for the prediction of the HSI, SSE, SZSE, TAIEX, NIKKEI, KOSPI indices. The authors of [153] used MODRL+LSTM method to predict Chinese stock-IF-IH-IC contract indices. The authors of [123] used stacked AEs to generate deep features using OCHL of the stock prices, technical indicators and macroeconomic conditions to feed to LSTM to predict the future stock prices.

Table 5: Index Forecasting Using Various Data

\begin{tabular}{|c|c|c|c|c|c|c|c|c|}
\hline Art. & Data Set & Period & Feature Set & Lag & Horizon & Method & $\begin{array}{l}\text { Performance } \\
\text { Criteria }\end{array}$ & Env. \\
\hline [114] & $\begin{array}{ll}\text { S\&P500 Index, } & 15 \\
\text { stocks in S\&P500 }\end{array}$ & $2006-2013$ & $\begin{array}{lr}\text { News } & \text { from } \\
\text { Reuters } & \text { and } \\
\text { Bloomberg } & \end{array}$ & - & - & $\mathrm{CNN}$ & Accuracy, MCC & - \\
\hline [116] & $\begin{array}{lll}\text { TWSE } & \text { index, } & 4 \\
\text { stocks in } & \text { TWSE }\end{array}$ & $2001-2017$ & $\begin{array}{l}\text { Technical indica- } \\
\text { tors, Index data, } \\
\text { News }\end{array}$ & $15 \mathrm{~d}$ & - & $\begin{array}{l}\text { CNN } \quad+ \\
\text { LSTM }\end{array}$ & RMSE, Profit & $\begin{array}{l}\text { Keras, } \\
\text { Python, } \\
\text { TALIB }\end{array}$ \\
\hline [123] & $\begin{array}{l}\text { CSI300, NIFTY50, } \\
\text { HSI, NIKKEI225, } \\
\text { S\&P500, DJIA }\end{array}$ & $2010-2016$ & $\begin{array}{l}\text { OCHLV, Techni- } \\
\text { cal Indicators }\end{array}$ & - & $1 \mathrm{~d}$ & $\begin{array}{l}\text { WT, Stacked } \\
\text { autoen- } \\
\text { coders, } \\
\text { LSTM }\end{array}$ & $\begin{array}{l}\text { MAPE, Correla- } \\
\text { tion coefficient, } \\
\text { THEIL-U }\end{array}$ & - \\
\hline$[126]$ & $\begin{array}{l}\text { FTSE100, OMXS } \\
30, \quad \text { SP500, Com- } \\
\text { modity, Forex }\end{array}$ & $1993-2017$ & $\begin{array}{l}\text { Technical indica- } \\
\text { tors }\end{array}$ & $60 \mathrm{~d}$ & $1 \mathrm{~d}$ & DNN, RNN & Accuracy, p-value & - \\
\hline [129] & $\begin{array}{l}\text { S\&P500, DOW30, } \\
\text { NASDAQ100, Com- } \\
\text { modity, } \\
\text { Bitcoin }\end{array}$ & $2003-2016$ & $\begin{array}{l}\text { Index data, Tech- } \\
\text { nical indicators }\end{array}$ & - & $\begin{array}{l}1 \mathrm{w} \\
1 \mathrm{~m}\end{array}$ & CNN & Accuracy & Tensorflow \\
\hline [130] & BSE, S\&P500 & $2004-2012$ & $\begin{array}{l}\text { Index data, tech- } \\
\text { nical indicators }\end{array}$ & $5 \mathrm{~d}$ & $1 \mathrm{~d} . .1 \mathrm{~m}$ & $\begin{array}{l}\mathrm{PSO}, \quad \mathrm{HM}- \\
\mathrm{RPSO}, \mathrm{DE}, \\
\text { RCEFLANN }\end{array}$ & RMSE, MAPE & - \\
\hline [135] & $\begin{array}{l}\text { HSI, SSE, SZSE, } \\
\text { TAIEX, NIKKEI, } \\
\text { KOSPI }\end{array}$ & $2010-2016$ & $\begin{array}{l}\text { Index data, vol- } \\
\text { ume, technical } \\
\text { indicators }\end{array}$ & $2 \mathrm{~d} . .512 \mathrm{~d}$ & $1 \mathrm{~d}$ & $\begin{array}{l}\text { LSTM with } \\
\text { wavelet } \\
\text { denoising }\end{array}$ & Accuracy, MAPE & - \\
\hline [137] & $\begin{array}{l}\text { DJIA, } \quad \text { FTSE, } \\
\text { NIKKEI, TAIEX }\end{array}$ & $1997-2008$ & $\begin{array}{l}\text { OCHLV, Techni- } \\
\text { cal indicators }\end{array}$ & $26 \mathrm{~d}$ & $1 \mathrm{~d}$ & RNN & $\begin{array}{l}\text { RMSE, } \text { MAE, } \\
\text { MAPE, THEIL- } \\
\text { U }\end{array}$ & $\mathrm{C}$ \\
\hline [150] & $\begin{array}{l}\text { Hedge fund monthly } \\
\text { return data }\end{array}$ & 1996-2015 & $\begin{array}{l}\text { Return, SR, } \\
\text { STD, Skewness, } \\
\text { Kurtosis, Omega } \\
\text { ratio, Fund alpha }\end{array}$ & $12 \mathrm{~m}$ & $\begin{array}{l}3 \mathrm{~m}, \\
6 \mathrm{~m} \\
12 \mathrm{~m}\end{array}$ & DNN & $\begin{array}{l}\text { Sharpe ratio, } \\
\text { Annual return, } \\
\text { Cum. return }\end{array}$ & - \\
\hline [151] &  & 2009-2014 & $\begin{array}{l}\text { FTSE100, } \\
\text { DJIA, GDAX, } \\
\text { NIKKEI225, } \\
\text { EUR/USD, Gold }\end{array}$ & $\begin{array}{l}1 \mathrm{~d} \\
2 \mathrm{~d} \\
5 \mathrm{~d} \\
10 \mathrm{~d}\end{array}$ & $1 \mathrm{~d}$ & $\begin{array}{l}\text { GASVR, } \\
\text { LSTM }\end{array}$ & $\begin{array}{l}\text { Return, volatil- } \\
\text { ity, SR, Accuracy }\end{array}$ & Tensorflow \\
\hline [152] & $\begin{array}{l}\text { Daily return ratio of } \\
\text { HS300 index }\end{array}$ & $2004-2018$ & $\begin{array}{l}\text { OCHLV, Techni- } \\
\text { cal indicators }\end{array}$ & - & - & $\begin{array}{l}\text { Market } \\
\text { Vector + } \\
\text { Tech. ind. } \\
+ \text { LSTM + } \\
\text { Attention }\end{array}$ & MSE, MAE & $\begin{array}{l}\text { Python, } \\
\text { Tensorflow }\end{array}$ \\
\hline [153] & $\begin{array}{l}\text { Chinese stock-IF- } \\
\text { IH-IC contract }\end{array}$ & $2016-2017$ & $\begin{array}{l}\text { Decisions for in- } \\
\text { dex change }\end{array}$ & $240 \mathrm{~min}$ & $1 \mathrm{~min}$ & MODRL+LST & $\begin{array}{l}\text { IProfit and loss, } \\
\text { SR }\end{array}$ & - \\
\hline
\end{tabular}


Table 5: Index Forecasting Using Various Data

\begin{tabular}{|c|c|c|c|c|c|c|c|c|}
\hline Art. & Data Set & Period & Feature Set & Lag & Horizon & Method & $\begin{array}{l}\text { Performance } \\
\text { Criteria }\end{array}$ & Env. \\
\hline [154] & HS300 & $2015-2017$ & $\begin{array}{l}\text { Social media } \\
\text { news, Index data }\end{array}$ & $1 d$ & $1 d$ & $\begin{array}{l}\text { RNN-Boost } \\
\text { with LDA }\end{array}$ & $\begin{array}{l}\text { Accuracy, MAE, } \\
\text { MAPE, RMSE }\end{array}$ & $\begin{array}{l}\text { Python, } \\
\text { Scikit- } \\
\text { learn }\end{array}$ \\
\hline
\end{tabular}

Besides, different CNN implementations with various data (technical indicators, news, index data) were used in the literature. In [129], CNN and index data, technical indicators were used for the S\&P500, DOW30, NASDAQ100 indices and Commodity, Forex, Bitcoin prices. In [114], CNN model with news from Reuters and Bloomberg were used for the prediction of S\&P500 Index and 15 stocks' prices in S\&P500. In [116], CNN + LSTM and technical indicators, index data, news were used for the forecasting of Taiwan Stock Exchange (TWSE) index and 4 stocks' prices in TWSE.

In addition, there were some novel methods proposed for the index forecasting. The authors of [130] used RNN models, Recurrent Computationally Efficient Functional Link Neural Network (RCEFLANN) and Functional Link Neural network (FLANN), with their weights optimized using various EA like Particle Swarm Optimization (PSO), HMRPSO and PSO for time series forecasting. The authors of [154] used social media news to predict the index price and index direction with RNN-Boost with Latent Dirichlet Allocation (LDA) features.

\subsection{Commodity Price Forecasting}

There were a number of studies particularly focused on the price prediction of any given commodity, such as gold, silver, oil, copper, etc. With increasing number of commodities that are available for public trading through online stock exchanges, interest in this topic will likely grow in the following years.

In the literature, there were different methods that were used for commodity price forecasting. DNN, RNN, FDDR, CNN were the most used models to predict the commodity prices. Table 6 provides the details about the commodity price forecasting studies with DL.

In [129], the authors used CNN for predicting the next week and next month price directional movement. Meanwhile, RNN and LSTM models were used in some of the commodity forecasting studies. In [155], DNN was used for Commodity forecasting. In [126], different datasets (Commodity, forex, index) were used as datasets. DNN and RNN were used to predict the prices of the time series data. Technical indicators were used as the feature set which consist of Relative Strength Index (RSI), Williams Percent Range (William\%R), Commodity Channel Index (CCI), Percentage Price Oscillator (PPOSC), momentum, Exponential Moving Average (EMA). In [156], the authors used Elman RNN to predict COMEX copper spot price (through New York Mercantile Exchange (NYMEX)) from daily close prices.

Hybrid and novel models were adapted in some studies. In [157], FNN and Stacked Denoising Autoencoders (SDAE) deep models were compared against Support Vector Regressor (SVR), Random Walk (RW) and Markov Regime Switching (MRS) models for WTI oil price forecasting. As performance criteria, accuracy, Mean Absolute Percentage Error (MAPE), 
Root Mean Square Error (RMSE) were used. In [158], authors tried to predict WTI crude oil prices using several models including combinations of DBN, LSTM, Autoregressive Moving Average (ARMA) and RW. MSE was used as the performance criteria. In [141], the authors used FDDR for stock price prediction and trading signal generation. They combined DNN and RL. Profit, return, SR, profit-loss curves were used as the performance criteria.

Table 6: Commodity Price Forecasting

\begin{tabular}{|c|c|c|c|c|c|c|c|c|}
\hline Art. & Data Set & Period & Feature Set & Lag & Horizon & Method & $\begin{array}{l}\text { Performance } \\
\text { Criteria }\end{array}$ & Env. \\
\hline [129] & $\begin{array}{l}\text { S\&P500, DOW30, } \\
\text { NASDAQ100, Com- } \\
\text { modity, } \\
\text { Bitcoin }\end{array}$ & $2003-2016$ & $\begin{array}{l}\text { Price data, Tech- } \\
\text { nical indicators }\end{array}$ & - & $\begin{array}{l}1 \mathrm{w} \\
1 \mathrm{~m}\end{array}$ & $\mathrm{CNN}$ & Accuracy & Tensorflow \\
\hline [155] & $\begin{array}{l}\text { Commodity, FX fu- } \\
\text { ture, ETF }\end{array}$ & 1991-2014 & Price Data & $100^{*} 5 \mathrm{~min}$ & $5 \mathrm{~min}$ & DNN & $\begin{array}{l}\text { SR, capability ra- } \\
\text { tio, return }\end{array}$ & $\begin{array}{l}\text { C }++, \\
\text { Python }\end{array}$ \\
\hline [126] & $\begin{array}{l}\text { FTSE100, OMX30, } \\
\text { S\&P500, Commod- } \\
\text { ity, Forex }\end{array}$ & $1993-2017$ & $\begin{array}{l}\text { Technical indica- } \\
\text { tors }\end{array}$ & $60 d$ & $1 \mathrm{~d}$ & DNN, RNN & Accuracy, p-value & - \\
\hline$[156]$ & $\begin{array}{l}\text { Copper prices from } \\
\text { NYMEX }\end{array}$ & $2002-2014$ & Price data & - & - & Elman RNN & RMSE & $\mathrm{R}$ \\
\hline$[157]$ & WTI crude oil price & 1986-2016 & Price data & $1 \mathrm{~m}$ & $1 \mathrm{~m}$ & $\begin{array}{l}\text { SDAE, Boot- } \\
\text { strap aggre- } \\
\text { gation }\end{array}$ & $\begin{array}{l}\text { Accuracy, } \\
\text { MAPE, RMSE }\end{array}$ & Matlab \\
\hline [158] & $\begin{array}{lll}\text { WTI } & \text { Crude } & \text { Oil } \\
\text { Prices } & & \end{array}$ & $2007-2017$ & Price data & - & - & $\begin{array}{ll}\text { ARMA }+ \\
\text { DBN, RW }+ \\
\text { LSTM }\end{array}$ & MSE & $\begin{array}{l}\text { Python, } \\
\text { Keras, } \\
\text { Tensorflow }\end{array}$ \\
\hline$[141]$ & $\begin{array}{l}300 \text { stocks from } \\
\text { SZSE, Commodity }\end{array}$ & $2014-2015$ & Price data & - & - & $\begin{array}{l}\text { FDDR, DNN } \\
+\mathrm{RL}\end{array}$ & $\begin{array}{lr}\begin{array}{l}\text { Profit, } \\
\text { SR, } \\
\text { curves }\end{array} & \text { profit-losn, } \\
\end{array}$ & Keras \\
\hline
\end{tabular}

\subsection{Volatility Forecasting}

Volatility is directly related with the price variations in a given time period and is mostly used for risk assesment and asset pricing. Some researchers implemented models for accurately forecasting the underlying volatility of any given asset.

In the literature, there were different methods that were used for volatility forecasting. LSTM, RNN, CNN, MM, Generalised Auto-Regressive Conditional Heteroscedasticity (GARCH) models were shown as some of these methods. Table 7 summarizes the studies that were focused on volatility forecasting. In Table 7, different methods/models are also represented as three sub-groups: CNN model; RNN and LSTM models; hybrid and novel models.

CNN model was used in one volatility forecasting study based on HFT data [159].

Meanwhile, RNN and LSTM models were used in some of the researches. In [160], the authors used financial time series data to predict volatility changes with Markov Models and Elman RNN for profitable straddle options trading. The authors of [161] used the price data and different types of Google Domestic trends with LSTM. The authors of [162] used CSI300, 28 words of the daily search volume based on Baidu as the dataset with LSTM to predict the index volatility. The authors of [163] developed several LSTM models integrated with GARCH for the prediction of volatility.

Hybrid and novel approaches were also adapted in some of the researches. In [164], RMDN with a GARCH (RMDN-GARCH) model was proposed. In addition, several models 
including traditional forecasting models and DL models were compared for the estimation of volatility. The authors of [149] proposed a novel method that is called HAR with a GASVR (HAR-GASVR) for volatility index forecasting.

Table 7: Volatility Forecasting

\begin{tabular}{|c|c|c|c|c|c|c|c|c|}
\hline Art. & Data Set & Period & Feature Set & Lag & Horizon & Method & $\begin{array}{l}\text { Performance } \\
\text { Criteria }\end{array}$ & Env. \\
\hline [159] & $\begin{array}{l}\text { London Stock Ex- } \\
\text { change }\end{array}$ & $2007-2008$ & $\begin{array}{l}\text { Limit order book } \\
\text { state, trades, } \\
\text { buy/sell orders, } \\
\text { order deletions }\end{array}$ & - & - & $\mathrm{CNN}$ & Accuracy, kappa & Caffe \\
\hline [160] & $\begin{array}{l}\text { DAX, FTSE100, } \\
\text { call/put options }\end{array}$ & 1991-1998 & Price data & $*$ & $*$ & MM, RNN & $\begin{array}{l}\text { Ewa-measure, } \\
\text { iv, daily profits' } \\
\text { mean and std }\end{array}$ & - \\
\hline [161] & S\&P 500 & $2004-2015$ & $\begin{array}{l}\text { Price data, } 25 \\
\text { Google Domestic } \\
\text { trend dimensions }\end{array}$ & - & $1 d$ & LSTM & MAPE, RMSE & - \\
\hline [162] & $\begin{array}{l}\text { CSI } 300,28 \text { words of } \\
\text { the daily search vol- } \\
\text { ume based on Baidu }\end{array}$ & 2006-2017 & $\begin{array}{l}\text { Price data and } \\
\text { text }\end{array}$ & $5 \mathrm{~d}$ & $5 \mathrm{~d}$ & LSTM & MSE, MAPE & $\begin{array}{l}\text { Python, } \\
\text { Keras }\end{array}$ \\
\hline [163] & $\begin{array}{l}\text { KOSPI200, Korea } \\
\text { Treasury Bond } \\
\text { interest rate, AA- } \\
\text { grade corporate } \\
\text { bond interest rate, } \\
\text { gold, crude oil }\end{array}$ & $2001-2011$ & Price data & $22 \mathrm{~d}$ & $1 d$ & $\begin{array}{ll}\text { LSTM } & + \\
\text { GARCH }\end{array}$ & $\begin{array}{l}\text { MAE, MSE, } \\
\text { HMAE, HMSE }\end{array}$ & - \\
\hline [164] & $\begin{array}{ll}\text { DEM/GBP } & \text { ex- } \\
\text { change rate }\end{array}$ & - & Returns & - & - & $\begin{array}{l}\text { RMDN- } \\
\text { GARCH }\end{array}$ & $\begin{array}{l}\text { NMSE, NMAE, } \\
\text { HR, WHR }\end{array}$ & - \\
\hline [149] & VIX, VXN, VXD & $2002-2014$ & $\begin{array}{l}\text { First five autore- } \\
\text { gressive lags }\end{array}$ & $5 \mathrm{~d}$ & $\begin{array}{l}1 \mathrm{~d}, \\
22 \mathrm{~d}\end{array}$ & $\begin{array}{l}\text { HAR- } \\
\text { GASVR }\end{array}$ & MAE, RMSE & - \\
\hline
\end{tabular}

\subsection{Bond Price Forecasting}

Some financial experts follow the changes in the bond prices to analyze the state of the economy, claiming bond prices represent the health of the economy better than the stock market [165]. Historically, long term rates are higher than the short term rates under normal economic expansion times, whereas just before recessions short term rates pass the long term rates, i.e. the inverted yield curve. Hence, accurate bond price prediction is very useful. However, DL implementations for bond price prediction is very scarce. In one study [166], excess bond return was predicted using several ML models including RF, AE and PCA network and a 2-3-4-layer DFNN. 4 layer NN outperformed the other models.

\subsection{Forex Price Forecasting}

Foreign exchange market has the highest volume among all existing financial markets in the world. It is open $24 / 7$ and trillions of dollars worth of foreign exhange transactions happen in a single day. According to the Bank for International Settlements, foreign-exchange trading had a volume of more than 5 trillion USD a day [167]. In addition, there are a large number of online forex trading platforms that provide leveraged transaction opportunities to their subscribers. As a result, there is a huge interest for profitable trading strategies by traders. Hence, there were a number of forex forecasting and trading studies that were based on DL models. Since most of the global financial transactions were based on US 
Dollar, almost all forex prediction research papers include USD in their analyses. However, depending on regional differences and intended research focus, various models were developed accordingly.

In the literature, there were different methods that were used for forex price forecasting. RNN, LSTM, CNN, DBN, DNN, AE, MLP methods were shown as some of these methods. Table 8 provides details about these implementations. In Table 8 , different methods/models are listed as four sub-groups: Continuous-valued Deep Belief Networks (CDBN), DBN, DBN+RBM, and AE models; DNN, RNN, Psi-Sigma Network (PSN), and LSTM models; CNN models; hybrid models.

CDBN, DBN, DBN+RBM, and AE models were used in some of the studies. In [168], Fuzzy information granulation integrated with CDBN was applied for predicting EUR/USD and GBU/USD exchange rates. They extended DBN with Continuous Restricted Boltzman machine (CRBM) to improve the performance. In [169], weekly GBP/USD and INR/USD prices were predicted, whereas in [170], CNY/USD and INR/USD was the main focus. In both cases, DBN was compared with FFNN. Similarly, the authors in [171] implemented several different DBN networks to predict weekly GBP/USD, BRL/USD and INR/USD exchange rate returns. The researchers in [172] combined Stacked AE and SVR for predicting 28 normalized currency pairs using the time series data of (USD, GBP, EUR, JPY, AUD, $\mathrm{CAD}, \mathrm{CHF}$ ).

DNN, RNN, PSN, and LSTM models were preferred in some of the researches. In [155], multiple DMLP models were developed for predicting AD and BP futures using 5minute data in a 130 day period. The authors of [173] used MLP, RNN, GP and other ML techniques along with traditional regression methods for also predicting EUR/USD time series. They also integrated Kalman filter, LASSO operator and other models to further improve the results in [174]. They further extended their analyses by including PSN and providing comparisons along with traditional forecasters like ARIMA, RW and STAR [175]. To improve the performance they also integrated hybrid time-varying volatility leverage. In [176], the authors implemented RMB exchange rate forecasting against JPY, HKB, EUR and USD by comparing RW, RNN and FFNN performances. In [177], the authors predicted various Forex time series and created portfolios consisted of these investments. Each network used LSTM (RNN EVOLINO) and different risk appetites for users have been tested. The authors of [178] also used EVOLINO RNN + orthogonal input data for predicting USD/JPY and XAU/USD prices for different periods.

Different CNN models were used in some of the studies. In [179], EUR/USD was once again forecasted using multiple DL models including MLP, CNN, RNN and Wavelet+CNN. The authors of [180] implemented forex trading (GBP/PLN) using several different input parameters on a multi-agent based trading environment. One of the agents was using $\mathrm{AE}+\mathrm{CNN}$ as the prediction model and outperformed all other models.

Hybrid models were also adapted in some of the researches. The authors of [148] developed several (TAR-VEC-RHE) models for predicting monthly returns for TRY/USD and compared model performances. In [164], the authors compared several models including traditional forecasting models and DL models for DEM/GBP prediction. The authors in [124] predicted AUD, CHF, MAX and BRL against USD currency time series data using 
LRNFIS and compared it with different models. Meanwhile, instead of using LMS based error minimization during the learning, they used FHSO.

Table 8: Forex Price Forecasting

\begin{tabular}{|c|c|c|c|c|c|c|c|c|}
\hline Art. & Data Set & Period & Feature Set & Lag & Horizon & Method & $\begin{array}{l}\text { Performance } \\
\text { Criteria }\end{array}$ & Env. \\
\hline [168] & $\begin{array}{l}\text { EUR/USD, } \\
\text { GBP/USD }\end{array}$ & $2009-2012$ & Price data & $*$ & $1 \mathrm{~d}$ & CDBN-FG & Profit & - \\
\hline [169] & $\begin{array}{l}\text { GBP/USD, } \\
\text { INR/USD }\end{array}$ & $1976-2003$ & Price data & $10 \mathrm{w}$ & $1 \mathrm{w}$ & $\mathrm{DBN}$ & $\begin{array}{lr}\text { RMSE, } & \text { MAE, } \\
\text { MAPE, } & \text { DA, } \\
\text { PCC } & \end{array}$ & - \\
\hline$[170]$ & CNY/USD,INR/USD & $1997-2016$ & Price data & - & $1 \mathrm{w}$ & $\mathrm{DBN}$ & $\begin{array}{l}\text { MAPE, } \\
\text { squared }\end{array}$ & - \\
\hline$[171]$ & $\begin{array}{l}\text { GBP/USD, } \\
\mathrm{BRL} / \mathrm{USD} \\
\mathrm{INR} / \mathrm{USD}\end{array}$ & $1976-2003$ & Price data & $10 \mathrm{w}$ & $1 \mathrm{w}$ & $\mathrm{DBN}+\mathrm{RBM}$ & $\begin{array}{l}\text { RMSE, MAE, } \\
\text { MAPE, accuracy, } \\
\text { PCC }\end{array}$ & - \\
\hline$[172]$ & $\begin{array}{lr}\text { Combination } & \text { of } \\
\text { USD, GBP, EUR, } \\
\text { JPY, AUD, CAD, } \\
\text { CHF }\end{array}$ & 2009-2016 & Price data & - & - & $\begin{array}{l}\text { Stacked } \\
+ \text { SVR }\end{array}$ & $\begin{array}{l}\text { MAE, } \\
\text { RMSE }\end{array}$ & Matlab \\
\hline [155] & $\begin{array}{l}\text { Commodity, FX fu- } \\
\text { ture, ETF }\end{array}$ & $1991-2014$ & Price Data & $100^{*} 5 \mathrm{~min}$ & $5 \min$ & $\overline{D N N}$ & $\begin{array}{l}\text { SR, capability ra- } \\
\text { tio, return }\end{array}$ & $\begin{array}{l}\mathrm{C}++, \\
\text { Python }\end{array}$ \\
\hline [126] & $\begin{array}{l}\text { FTSE100, OMX30, } \\
\text { S\&P500, Commod- } \\
\text { ity, Forex }\end{array}$ & 1993-2017 & $\begin{array}{l}\text { Technical indica- } \\
\text { tors }\end{array}$ & $60 d$ & $1 \mathrm{~d}$ & DNN, RNN & Accuracy, p-value & - \\
\hline$[173]$ & EUR/USD & $2001-2010$ & Close data & $11 \mathrm{~d}$ & $1 d$ & $\begin{array}{ll}\mathrm{RNN} & \text { and } \\
\text { more }\end{array}$ & $\begin{array}{l}\text { MAE, } \text { MAPE, } \\
\text { RMSE, THEIL-U }\end{array}$ & - \\
\hline$[174]$ & $\mathrm{EUR} / \mathrm{USD}$ & $2002-2010$ & Price data & $13 \mathrm{~d}$ & $1 d$ & $\begin{array}{l}\text { RNN, MLP, } \\
\text { PSN }\end{array}$ & $\begin{array}{l}\text { MAE, } \text { MAPE, } \\
\text { RMSE, THEIL-U }\end{array}$ & - \\
\hline$[175]$ & $\begin{array}{l}\mathrm{EUR} / \mathrm{USD} \\
\mathrm{EUR} / \mathrm{GBP} \\
\mathrm{EUR} / \mathrm{JPY} \\
\mathrm{EUR} / \mathrm{CHF}\end{array}$ & $1999-2012$ & Price data & $12 \mathrm{~d}$ & $1 \mathrm{~d}$ & $\begin{array}{l}\text { RNN, MLP, } \\
\text { PSN }\end{array}$ & $\begin{array}{l}\text { MAE, MAPE, } \\
\text { RMSE, THEIL-U }\end{array}$ & - \\
\hline$[176]$ & $\begin{array}{l}\text { RMB against USD, } \\
\text { EUR, JPY, HKD }\end{array}$ & $2006-2008$ & Price data & $10 \mathrm{~d}$ & $1 \mathrm{~d}$ & RNN, ANN & $\begin{array}{l}\text { RMSE, } \\
\text { MSE }\end{array}$ & - \\
\hline$[177]$ & $\begin{array}{l}\text { EUR/USD, } \\
\text { EUR/JPY, } \\
\text { USD/JPY, } \\
\text { EUR/CHF, } \\
\text { XAU/USD, } \\
\text { XAG/USD, QM, } \\
\text { QG }\end{array}$ & $2011-2012$ & Price data & - & - & Evolino RNN & $\begin{array}{l}\text { Correlation be- } \\
\text { tween predicted, } \\
\text { real values }\end{array}$ & - \\
\hline [178] & USD/JPY & $2009-2010$ & Price data, Gold & - & $5 \mathrm{~d}$ & $\begin{array}{l}\text { EVOLINO } \\
\text { RNN }+ \\
\text { orthogonal } \\
\text { input data }\end{array}$ & RMSE & - \\
\hline$[179]$ & S\&P500, EUR/USD & $1950-2016$ & Price data & $\begin{array}{l}30 \mathrm{~d}, \\
30 \mathrm{~d}^{*} \min \end{array}$ & $\begin{array}{l}1 \mathrm{~d}, \\
1 \mathrm{~min}\end{array}$ & Wavelet+CNN & $\begin{array}{l}\text { Accuracy, log- } \\
\text { loss }\end{array}$ & Keras \\
\hline [180] & $\begin{array}{l}\text { USD/GBP, } \\
\text { S\&P500, FTSE100, } \\
\text { oil, gold }\end{array}$ & 2016 & Price data & - & $5 \mathrm{~min}$ & $\mathrm{AE}+\mathrm{CNN}$ & $\begin{array}{l}\text { SR, \% volatility, } \\
\text { avg return/trans, } \\
\text { rate of return }\end{array}$ & $\mathrm{H} 2 \mathrm{O}$ \\
\hline [148] & ISE100, TRY/USD & $1987-2008$ & Price data & - & $\begin{array}{l}2 \mathrm{~d}, \\
4 \mathrm{~d}, \\
8 \mathrm{~d}, \\
12 \mathrm{~d}, \\
18 \mathrm{~d}\end{array}$ & $\begin{array}{l}\text { TAR-VEC- } \\
\text { MLP, TAR- } \\
\text { VEC-RBF, } \\
\text { TAR-VEC- } \\
\text { RHE }\end{array}$ & RMSE & - \\
\hline [164] & $\begin{array}{l}\text { DEM/GBP } \\
\text { change rate }\end{array}$ & - & Returns & - & - & $\begin{array}{l}\text { RMDN- } \\
\text { GARCH }\end{array}$ & $\begin{array}{l}\text { NMSE, NMAE, } \\
\text { HR, WHR }\end{array}$ & - \\
\hline$[124]$ & $\begin{array}{l}\text { S\&P500, } \\
\text { NIKKEI225, USD } \\
\text { Exchanges }\end{array}$ & $2011-2015$ & Price data & - & $\begin{array}{l}1 \mathrm{~d} \\
5 \mathrm{~d}, \\
7 \mathrm{~d} \\
10 \mathrm{~d}\end{array}$ & $\begin{array}{l}\text { LRNFIS with } \\
\text { FHSO }\end{array}$ & $\begin{array}{l}\text { RMSE, MAPE, } \\
\text { MAE }\end{array}$ & - \\
\hline
\end{tabular}




\subsection{Cryptocurrency Price Forecasting}

Since cryptocurrencies became a hot topic for discussion in the finance world, lots of studies and implementations started emerging in recent years. Most of the cryptocurrency studies were focused on price forecasting.

The rise of bitcoin from 1000 USD in January 2017 to 20,000 USD in January 2018 has attracted a lot of attention not only from the financial world, but also from ordinary people on the street. Recently, some papers have been published for price prediction and trading strategy development for bitcoin and other cryptocurrencies. Given the attention that the underlying technology has attracted, there is a great chance that some new studies will start appearing in the near future.

In the literature, DNN, LSTM, GRU, RNN, Classical methods (ARMA, ARIMA, Autoregressive Conditional Heteroscedasticity (ARCH), GARCH, etc) were used for cryptocurrency price forecasting. Table 9 tabulates the studies that utilize these methods. In [181], the author combined the opinion market and price prediction for cryptocurrency trading. Text mining combined with 2 models CNN and LSTM were used to extract the opinion. Bitcoin, Litecoin, StockTwits were used as the dataset. OCHLV of prices, technical indicators, and sentiment analysis were used as the feature set. In [182], the authors compared Bayesian optimized RNN, LSTM and ARIMA to predict bitcoin price direction. Sensitivity, specificity, precision, accuracy, RMSE were used as the performance metrics.

Table 9: Cryptocurrency Price Prediction

\begin{tabular}{|c|c|c|c|c|c|c|c|c|}
\hline Art. & Data Set & Period & Feature Set & Lag & Horizon & Method & $\begin{array}{l}\text { Performance } \\
\text { Criteria }\end{array}$ & Env. \\
\hline$[181]$ & $\begin{array}{l}\text { Bitcoin, Litecoin, } \\
\text { StockTwits }\end{array}$ & $2015-2018$ & $\begin{array}{l}\text { OCHLV, tech- } \\
\text { nical indicators, } \\
\text { sentiment analy- } \\
\text { sis }\end{array}$ & - & $\begin{array}{l}30 \mathrm{~min}, \\
4 \mathrm{~h}, 1 \mathrm{~d}\end{array}$ & $\begin{array}{l}\text { CNN, LSTM, } \\
\text { State Fre- } \\
\text { quency } \\
\text { Model }\end{array}$ & MSE & $\begin{array}{l}\text { Keras, } \\
\text { Tensorflow }\end{array}$ \\
\hline [182] & Bitcoin & $2013-2016$ & Price data & $100 d$ & $30 \mathrm{~d}$ & $\begin{array}{l}\text { Bayesian } \\
\text { optimized } \\
\text { RNN, LSTM }\end{array}$ & $\begin{array}{l}\text { Sensitivity, speci- } \\
\text { ficity, precision, } \\
\text { accuracy, RMSE }\end{array}$ & $\begin{array}{l}\text { Keras, } \\
\text { Python, } \\
\text { Hyperas }\end{array}$ \\
\hline
\end{tabular}

\subsection{Trend Forecasting}

Even though trend forecasting and price forecasting share the same input characteristics, some researchers prefer to predict the price direction of the asset instead of the actual price. This alters the nature of the problem from regression to classification and the corresponding performance metrics also change. However, it is worth to mention that these two approaches are not really different, the difference is in the interpretation of the output.

In the literature, there were different methods for trend forecasting. In this survey, we grouped the articles according to their feature set such as studies using only the raw time series data (only price data, OCHLV); studies using technical indicators \& price data \& fundamental data at the same time; studies using text mining techniques and studies using other various data. Table 10 tabulates the trend forecasting using only the raw time series data. 
Table 10: Trend Forecasting Using Only Raw Time Series Data

\begin{tabular}{|c|c|c|c|c|c|c|c|c|}
\hline Art. & Data Set & Period & Feature Set & Lag & Horizon & Method & $\begin{array}{l}\text { Performance } \\
\text { Criteria }\end{array}$ & Env. \\
\hline [183] & $\begin{array}{l}\text { S\&P500 stock in- } \\
\text { dexes }\end{array}$ & $1963-2016$ & Price data & $30 \mathrm{~d}$ & $1 d$ & $\mathrm{NN}$ & $\begin{array}{l}\text { Accuracy, preci- } \\
\text { sion, recall, F1- } \\
\text { score, AUROC }\end{array}$ & $\begin{array}{l}\mathrm{R}, \mathrm{H} 2 \mathrm{o}, \\
\text { Python, } \\
\text { Tensorflow }\end{array}$ \\
\hline [184] & $\begin{array}{l}\text { SPY ETF, } 10 \text { stocks } \\
\text { from S\&P } 500\end{array}$ & 2014-2016 & Price data & $60 \mathrm{~min}$ & $30 \mathrm{~min}$ & FNN & Cumulative gain & $\begin{array}{l}\text { MatConvNet } \\
\text { Matlab }\end{array}$ \\
\hline [142] & $\begin{array}{l}\text { Shanghai composite } \\
\text { index and SZSE }\end{array}$ & $1990-2016$ & OCHLV & $20 \mathrm{~d}$ & $1 \mathrm{~d}$ & $\begin{array}{l}\text { Ensembles of } \\
\text { ANN }\end{array}$ & Accuracy & - \\
\hline [185] & $\begin{array}{ll}10 \quad \text { stocks } & \text { from } \\
\text { S\&P } 500 & \end{array}$ & - & Price data & & & $\begin{array}{l}\text { TDNN, } \\
\text { RNN, PNN }\end{array}$ & $\begin{array}{l}\text { Missed } \quad \text { oppor- } \\
\text { tunities, false } \\
\text { alarms ratio }\end{array}$ & - \\
\hline$[186]$ & $\begin{array}{l}\text { GOOGL stock daily } \\
\text { price data }\end{array}$ & $2012-2016$ & $\begin{array}{l}\text { Time window } \\
\text { of } 30 \text { days of } \\
\text { OCHLV }\end{array}$ & $\begin{array}{l}22 \mathrm{~d}, \\
50 \mathrm{~d}, \\
70 \mathrm{~d}\end{array}$ & $*$ & $\begin{array}{l}\text { LSTM, GRU, } \\
\text { RNN }\end{array}$ & $\begin{array}{l}\text { Accuracy, } \\
\text { Logloss }\end{array}$ & $\begin{array}{l}\text { Python, } \\
\text { Keras }\end{array}$ \\
\hline [133] & $\begin{array}{l}\text { S\&P500, } \\
\text { Bovespa50, OMX30 }\end{array}$ & 2009-2017 & $\begin{array}{l}\text { Autoregressive } \\
\text { part of the price } \\
\text { data }\end{array}$ & $30 \mathrm{~d}$ & $1 . .15 \mathrm{~d}$ & LSTM & MSE, Accuracy & $\begin{array}{l}\text { Tensorflow, } \\
\text { Keras, R }\end{array}$ \\
\hline [187] & HSI, DAX, S\&P500 & 1991-2017 & Price data & - & $1 \mathrm{~d}$ & $\begin{array}{l}\text { GRU, GRU- } \\
\text { SVM }\end{array}$ & Daily return \% & $\begin{array}{l}\text { Python, } \\
\text { Tensorflow }\end{array}$ \\
\hline [188] & $\begin{array}{l}\text { Taiwan Stock Index } \\
\text { Futures }\end{array}$ & $2001-2015$ & OCHLV & $240 \mathrm{~d}$ & $1 . .2 \mathrm{~d}$ & $\begin{array}{lr}\text { CNN } r i t h \\
\text { GAF, MAM, } \\
\text { Candlestick }\end{array}$ & Accuracy & Matlab \\
\hline [189] & ETF and Dow30 & $1997-2007$ & Price data & & & $\begin{array}{l}\text { CNN with } \\
\text { feature imag- } \\
\text { ing }\end{array}$ & $\begin{array}{l}\text { Annualized } \\
\text { return }\end{array}$ & $\begin{array}{l}\text { Keras, } \\
\text { Tensorflow }\end{array}$ \\
\hline [190] & $\begin{array}{ll}\text { SSEC, NASDAQ, } \\
\text { S\&P500 }\end{array}$ & $2007-2016$ & Price data & $20 \mathrm{~min}$ & $7 \mathrm{~min}$ & EMD2FNN & $\begin{array}{l}\text { MAE, RMSE, } \\
\text { MAPE }\end{array}$ & - \\
\hline [191] & $\begin{array}{l}23 \text { cap stocks from } \\
\text { the OMX30 index in } \\
\text { Nasdaq Stockholm }\end{array}$ & $2000-2017$ & $\begin{array}{l}\text { Price data and } \\
\text { returns }\end{array}$ & $30 \mathrm{~d}$ & $*$ & $\mathrm{DBN}$ & MAE & $\begin{array}{l}\text { Python, } \\
\text { Theano }\end{array}$ \\
\hline
\end{tabular}

Different methods and models were used for trend forecasting. In Table 10, these are divided into three sub-groups: ANN, DNN, and FFNN models; LSTM, RNN, and Probabilistic NN models; novel methods. ANN, DNN, DFNN, and FFNN methods were used in some of the studies. In [183], NN with the price data were used for prediction of the trend of S\&P500 stock indices. The authors of [184] combined deep FNN with a selective trading strategy unit to predict the next price. The authors of [142] created an ensemble network of several Backpropagation and ADAM models for trend prediction.

In the literature, LSTM, RNN, Probabilistic Neural Network (PNN) methods with the raw time series data were also used for trend forecasting. In [185], the authors compared Timedelay Neural Network (TDNN), RNN and PNN for trend detection using 10 stocks from S\&P500. The authors of [186] compared 3 different RNN models (basic RNN, LSTM, GRU) to predict the movement of Google stock price. The authors of [133] used LSTM (and other classical forecasting techniques) to predict the trend of the stocks prices. In [187], GRU and GRU-SVM models were used for the trend of HSI, The Deutscher Aktienindex (DAX), S\&P500 indices.

There were also novel methods that used only the raw time series price/index data in the literature. The author of [188] proposed a method that used CNN with Gramian Angular Field (GAF), Moving Average Mapping (MAM), Candlestick with converted image data. In [189], a novel method, CNN with feature imaging was proposed for the prediction of the buy/sell/hold positions of the Exchange-Traded Funds (ETFs)' prices and Dow30 stocks' 
prices. The authors of [190] proposed a method that uses Empirical Mode Decomposition and Factorization Machine based Neural Network (EMD2FNN) models to forecast the stock close prices' direction accurately. In [191], DBN with the price data were used for the prediction of the trend of 23 large cap stocks from the OMX30 index.

Table 11: Trend Forecasting Using Technical Indicators \& Price Data \& Fundamental Data

\begin{tabular}{|c|c|c|c|c|c|c|c|c|}
\hline Art. & Data Set & Period & Feature Set & Lag & Horizon & Method & $\begin{array}{l}\text { Performance } \\
\text { Criteria }\end{array}$ & Env. \\
\hline [192] & KSE100 index & - & $\begin{array}{l}\text { Price data, sev- } \\
\text { eral fundamental } \\
\text { data }\end{array}$ & - & - & $\begin{array}{l}\text { ANN, } \\
\text { MLP, } \\
\text { DBNF, } \\
\text { DVM }\end{array}$ & Accuracy & - \\
\hline [193] & Stocks in Dow30 & $1997-2017$ & $\begin{array}{l}\text { RSI (Technical } \\
\text { Indicators) }\end{array}$ & $200 d$ & $1 d$ & $\begin{array}{ll}\text { DMLP } & \text { with } \\
\text { genetic } & \text { algo- } \\
\text { rithm } & \end{array}$ & $\begin{array}{l}\text { Annualized } \\
\text { return }\end{array}$ & $\begin{array}{l}\text { Spark ML- } \\
\text { lib, Java }\end{array}$ \\
\hline [194] & $\begin{array}{l}\text { SSE Composite In- } \\
\text { dex, FTSE100, Pin- } \\
\text { gAnBank }\end{array}$ & 1999-2016 & $\begin{array}{l}\text { Technical indi- } \\
\text { cators, OCHLV } \\
\text { price }\end{array}$ & $24 d$ & $1 d$ & RBM & Accuracy & - \\
\hline [195] & Dow30 stocks & $2012-2016$ & $\begin{array}{l}\text { Price data, sev- } \\
\text { eral technical in- } \\
\text { dicators }\end{array}$ & $40 \mathrm{~d}$ & - & LSTM & Accuracy & $\begin{array}{l}\text { Python, } \\
\text { Keras, } \\
\text { Tensor- } \\
\text { flow, } \\
\text { TALIB }\end{array}$ \\
\hline [196] & $\begin{array}{l}\text { Stock price from } \\
\text { IBOVESPA index }\end{array}$ & $2008-2015$ & $\begin{array}{l}\text { Technical indica- } \\
\text { tors, OCHLV of } \\
\text { price }\end{array}$ & - & $15 \mathrm{~min}$ & LSTM & $\begin{array}{l}\text { Accuracy, Preci- } \\
\text { sion, Recall, F1- } \\
\text { score, \% return, } \\
\text { Maximum draw- } \\
\text { down }\end{array}$ & Keras \\
\hline [197] & $\begin{array}{l}20 \text { stocks from NAS- } \\
\text { DAQ and NYSE }\end{array}$ & $2010-2017$ & $\begin{array}{l}\text { Price data, tech- } \\
\text { nical indicators }\end{array}$ & $5 \mathrm{~d}$ & $1 d$ & $\begin{array}{l}\text { LSTM, GRU, } \\
\text { SVM, XG- } \\
\text { Boost }\end{array}$ & Accuracy & $\begin{array}{l}\text { Keras, } \\
\text { Tensor- } \\
\text { flow, } \\
\text { Python }\end{array}$ \\
\hline [198] & $17 \mathrm{ETF}$ & $2000-2016$ & $\begin{array}{l}\text { Price data, tech- } \\
\text { nical indicators }\end{array}$ & $28 \mathrm{~d}$ & $1 \mathrm{~d}$ & CNN & $\begin{array}{l}\text { Accuracy, MSE, } \\
\text { Profit, AUROC }\end{array}$ & $\begin{array}{l}\text { Keras, } \\
\text { Tensorflow }\end{array}$ \\
\hline [199] & $\begin{array}{lrr}\text { Stocks } & \text { in } & \text { Dow30 } \\
\text { and } 9 & \text { Top } & \text { Volume } \\
\text { ETF } & & \end{array}$ & $1997-2017$ & $\begin{array}{l}\text { Price data, tech- } \\
\text { nical indicators }\end{array}$ & $20 \mathrm{~d}$ & $1 \mathrm{~d}$ & $\begin{array}{l}\text { CNN with } \\
\text { feature imag- } \\
\text { ing }\end{array}$ & $\begin{array}{l}\text { Recall, precision, } \\
\text { F1-score, annual- } \\
\text { ized return }\end{array}$ & $\begin{array}{l}\text { Python, } \\
\text { Keras, } \\
\text { Tensor- } \\
\text { flow, Java }\end{array}$ \\
\hline [200] & $\begin{array}{l}\text { Borsa Istanbul } 100 \\
\text { Stocks }\end{array}$ & $2011-2015$ & $\begin{array}{l}75 \text { technical in- } \\
\text { dicators, OCHLV } \\
\text { of price }\end{array}$ & - & $1 \mathrm{~h}$ & $\mathrm{CNN}$ & Accuracy & Keras \\
\hline
\end{tabular}

In the literature, some of the studies used technical indicators \& price data \& fundamental data at the same time. Table 11 tabulates the trend forecasting papers using technical indicators, price data, fundamental data. In addition, these studies are clustered into three sub-groups: ANN, MLP, DBN, and RBM models; LSTM and GRU models; novel methods. ANN, MLP, DBN, and RBM methods were used with technical indicators, price data and fundamental data in some of the studies. In [192], several classical, ML models and DBN were compared for trend forecasting. In [193], technical analysis indicator's (RSI) buy \& sell limits were optimized with GA which was used for buy-sell signals. After optimization, DMLP was also used for function approximation. The authors of [194] used technical analysis parameters, OCHLV of prices and RBM for stock trend prediction.

Besides, LSTM and GRU methods with technical indicators \& price data \& fundamental data were also used in some of the papers. In [195], the crossover and Moving Average Convergence and Divergence (MACD) signals were used to predict the trend of the Dow 30 
stocks prices. The authors of [196] used LSTM for stock price movement estimation. The author of [197] used stock prices, technical analysis features and four different ML Models (LSTM, GRU, SVM and eXtreme Gradient Boosting (XGBoost)) to predict the trend of the stocks prices.

In addition, there were also novel and new methods that used CNN with the price data and technical indicators. The authors of [198] converted the time series of price data to 2-dimensional images using technical analysis and classified them with deep CNN. Similarly, the authors of [199] also proposed a novel technique that converted financial time series data that consisted of technical analysis indicator outputs to 2-dimensional images and classified these images using CNN to determine the trading signals. The authors of [200] proposed a method that used CNN with correlated features combined together to predict the trend of the stocks prices.

Besides, there were also studies that used text mining techniques in the literature. Table 12 tabulates the trend forecasting papers using text mining techniques. Different methods/models are represented within four sub-groups in that table: DNN, DMLP, and CNN with text mining models; GRU model; LSTM, CNN, and LSTM+CNN models; novel methods. In the first group of studies, DNN, DMLP, CNN with text mining were used for trend forecasting. In [201], the authors used different models that included Hidden Markov Model (HMM), DMLP and CNN using Twitter moods to predict the next days' move. In [202], the authors used the combination of text mining and word embeddings to extract information from financial news and DNN model for prediction of the stock trends.

Moreover, GRU methods with text mining techniques were also used for trend forecasting. The authors of [203] used financial news from Reuters, Bloomberg and stock prices data and Bidirectional Gated Recurrent Unit (Bi-GRU) model to predict the stock movements in the future. The authors of [204] used Stock2Vec and Two-stream GRU (TGRU) models to generate input data from financial news and stock prices. Then, they used the sign difference between the previous close and next open for the classification of the stock prices. The results were better than the state-of-the-art models.

LSTM, CNN and LSTM + CNN models were also used for trend forecasting. The authors of [205] combined news data with financial data to classify the stock price movement and assessed them with certain factors. They used LSTM model as the NN architecture. The authors of [206] proposed a novel method that used character-based neural language model using financial news and LSTM for trend prediction. In [207], sentiment/mood prediction and price prediction based on sentiment, price prediction with text mining and DL models (LSTM, NN, CNN) were used for trend forecasting. The authors of [208] proposed a method that used two separate LSTM networks to construct an ensemble network. One of the LSTM models was used for word embeddings with word2Vec to create a matrix information as input to CNN. The other one was used for price prediction using technical analysis features and stock prices.

In the literature, there were also novel and different methods to predict the trend of the time series data. In [209], the authors proposed a novel method that uses a combination of RBM, DBN and word embedding to create word vectors for RNN-RBM-DBN network to predict the trend of stock prices. The authors of [210] proposed a novel method (called 
DeepClue) that visually interpretted text-based DL models in predicting stock price movements. In their proposed method, financial news, charts and social media tweets were used together to predict the stock price movement. The authors of [211] proposed a method that performed information fusion from several news and social media sources to predict the trend of the stocks. The authors of [212] proposed a novel method that used text mining techniques and Hybrid Attention Networks based on financial news for the forecast of the trend of stocks. The authors of [213] combined technical analysis and sentiment analysis of social media (related financial topics) and created Deep Random Subspace Ensembles (DRSE) method for classification. The authors of [214] proposed a method that used Deep Neural Generative Model (DGM) with news articles using Paragraph Vector algorithm to create the input vector for the prediction of the trend of stocks. The authors of [215] implemented intraday stock price direction classification using financial news and stocks prices.

Table 12: Trend Forecasting Using Text Mining Techniques

\begin{tabular}{|c|c|c|c|c|c|c|c|c|}
\hline Art. & Data Set & Period & Feature Set & Lag & Horizon & Method & $\begin{array}{l}\text { Performance } \\
\text { Criteria }\end{array}$ & Env. \\
\hline [201] & $\begin{array}{l}\text { S\&P500, } \\
\text { Composite, DJIA, } \\
\text { NASDAQ Compos- } \\
\text { ite }\end{array}$ & $2009-2011$ & $\begin{array}{l}\text { Twitter moods, } \\
\text { index data }\end{array}$ & $7 \mathrm{~d}$ & $1 \mathrm{~d}$ & $\mathrm{DNN}, \mathrm{CNN}$ & Error rate & $\begin{array}{l}\text { Keras, } \\
\text { Theano }\end{array}$ \\
\hline [202] & $\begin{array}{lr}\text { News from Reuters } \\
\text { and Bloomberg, } \\
\text { Historical stock } \\
\text { security data }\end{array}$ & 2006-2013 & News, price data & $5 \mathrm{~d}$ & $1 d$ & DNN & Accuracy & - \\
\hline [203] & $\begin{array}{l}\text { News from Reuters, } \\
\text { Bloomberg }\end{array}$ & 2006-2013 & $\begin{array}{l}\text { Financial news, } \\
\text { price data }\end{array}$ & - & $\begin{array}{l}1 \mathrm{~d}, 2 \mathrm{~d} \\
5 \mathrm{~d}, 7 \mathrm{~d}\end{array}$ & Bi-GRU & Accuracy & $\begin{array}{l}\text { Python, } \\
\text { Keras }\end{array}$ \\
\hline [204] & $\begin{array}{l}\text { News about Apple, } \\
\text { Airbus, Amazon } \\
\text { from Reuters, } \\
\text { Bloomberg, S\&P500 } \\
\text { stock prices }\end{array}$ & 2006-2013 & $\begin{array}{l}\text { Price data, news, } \\
\text { technical indica- } \\
\text { tors }\end{array}$ & - & - & $\begin{array}{l}\text { Two-stream } \\
\text { GRU, } \\
\text { stock2vec }\end{array}$ & $\begin{array}{l}\text { Accuracy, preci- } \\
\text { sion, AUROC }\end{array}$ & $\begin{array}{l}\text { Keras, } \\
\text { Python }\end{array}$ \\
\hline [205] & $\begin{array}{lc}\text { NIFTY50 } & \text { In- } \\
\text { dex, } & \text { NIFTY } \\
\text { Bank/Auto/IT/Energy } \\
\text { Index, News }\end{array}$ & 2013-2017 & Index data, news & $\begin{array}{l}1 \mathrm{~d}, 2 \mathrm{~d} \\
5 \mathrm{~d}\end{array}$ & $1 \mathrm{~d}$ & LSTM & MCC, Accuracy & - \\
\hline [206] & $\begin{array}{lr}\text { News from Reuters, } \\
\text { Bloomberg, } & \text { stock } \\
\text { price/index } & \text { data } \\
\text { from S\&P500 } & \end{array}$ & 2006-2013 & $\begin{array}{l}\text { News and sen- } \\
\text { tences }\end{array}$ & - & $1 \mathrm{~h}, 1 \mathrm{~d}$ & LSTM & Accuracy & - \\
\hline [207] & $\begin{array}{l}30 \text { DJIA stocks, } \\
\text { S\&P 500, DJI, news } \\
\text { from Reuters }\end{array}$ & $2002-2016$ & $\begin{array}{l}\text { Price data and } \\
\text { features from } \\
\text { news articles }\end{array}$ & $1 \mathrm{~m}$ & $1 \mathrm{~d}$ & $\begin{array}{l}\text { LSTM, NN, } \\
\text { CNN and } \\
\text { word2vec }\end{array}$ & Accuracy & VADER \\
\hline [208] & $\begin{array}{l}\text { APPL from S\&P500 } \\
\text { and news from } \\
\text { Reuters }\end{array}$ & 2011-2017 & $\begin{array}{l}\text { News, OCHLV, } \\
\text { Technical indica- } \\
\text { tors }\end{array}$ & - & $1 \mathrm{~d}$ & $\begin{array}{l}\text { CNN }+ \\
\text { LSTM, } \\
\text { CNN+SVM }\end{array}$ & $\begin{array}{l}\text { Accuracy, F1- } \\
\text { score }\end{array}$ & Tensorflow \\
\hline [209] & $\begin{array}{l}\text { News, Nikkei Stock } \\
\text { Average and 10- } \\
\text { Nikkei companies }\end{array}$ & 1999-2008 & News, MACD & - & $1 d$ & $\begin{array}{l}\mathrm{RNN}, \\
\mathrm{RBM}+\mathrm{DBN}\end{array}$ & $\begin{array}{l}\text { Accuracy, } \quad \text { P- } \\
\text { value }\end{array}$ & - \\
\hline [210] & $\begin{array}{l}\text { News from Reuters } \\
\text { and Bloomberg for } \\
\text { S\&P500 stocks }\end{array}$ & 2006-2015 & $\begin{array}{l}\text { Financial news, } \\
\text { price data }\end{array}$ & $1 \mathrm{~d}$ & $1 \mathrm{~d}$ & DeepClue & Accuracy & $\begin{array}{l}\text { Dynet } \\
\text { software }\end{array}$ \\
\hline [211] & $\begin{array}{ll}\text { Price data, } & \text { index } \\
\text { data, news, } & \text { social } \\
\text { media data } & \\
\end{array}$ & 2015 & $\begin{array}{l}\text { Price data, news } \\
\text { from articles and } \\
\text { social media }\end{array}$ & $1 \mathrm{~d}$ & $1 \mathrm{~d}$ & $\begin{array}{l}\text { Coupled ma- } \\
\text { trix and ten- } \\
\text { sor }\end{array}$ & Accuracy, MCC & Jieba \\
\hline$[212]$ & $\begin{array}{l}\text { News and Chinese } \\
\text { stock data }\end{array}$ & 2014-2017 & $\begin{array}{l}\text { Selected words in } \\
\text { a news }\end{array}$ & $10 \mathrm{~d}$ & $1 d$ & HAN & $\begin{array}{ll}\text { Accuracy, } & \text { An- } \\
\text { nual return }\end{array}$ & - \\
\hline
\end{tabular}


Table 12: Trend Forecasting Using Text Mining Techniques

\begin{tabular}{|c|c|c|c|c|c|c|c|c|}
\hline Art. & Data Set & Period & Feature Set & Lag & Horizon Method & $\begin{array}{l}\text { Performa } \\
\text { Criteria }\end{array}$ & nce & Env. \\
\hline [213] & $\begin{array}{l}\text { Sina Weibo, Stock } \\
\text { market records }\end{array}$ & $2012-2015$ & $\begin{array}{l}\text { Technical indica- } \\
\text { tors, sentences }\end{array}$ & - & DRSE & $\begin{array}{l}\text { F1-score, } \\
\text { cision, } \\
\text { accuracy, } \\
\text { ROC }\end{array}$ & $\begin{array}{r}\text { pre- } \\
\text { recall, } \\
\text { AU- }\end{array}$ & Python \\
\hline$[214]$ & $\begin{array}{l}\text { Nikkei225, S\&P500, } \\
\text { news from Reuters } \\
\text { and Bloomberg }\end{array}$ & $2001-2013$ & $\begin{array}{l}\text { Price data and } \\
\text { news }\end{array}$ & $1 d$ & $\overline{D G M}$ & $\begin{array}{l}\text { Accuracy, } \\
\text { \%profit }\end{array}$ & MCC, & - \\
\hline$[215]$ & $\begin{array}{l}\text { News, stock prices } \\
\text { from Hong Kong } \\
\text { Stock Exchange }\end{array}$ & 2001 & $\begin{array}{lr}\text { Price data } & \text { and } \\
\text { TF-IDF } & \text { from } \\
\text { news } & \end{array}$ & $60 \mathrm{~min}$ & $\begin{array}{r}(1 . .6)^{*} 5 \mathrm{miELM}, \quad \text { DLR, } \\
\text { PCA, BELM, } \\
\text { KELM, NN }\end{array}$ & Accuracy & & Matlab \\
\hline
\end{tabular}

Moreover, there were also studies that used different data variations in the literature. Table 13 tabulates the trend forecasting papers using these various data clustered into two sub-groups: LSTM, RNN, GRU models; CNN model.

LSTM, RNN, GRU methods with various data representations were used in some trend forecasting papers. In [216], the authors used the limit order book time series data and LSTM method for trend prediction. The authors of [217] proposed a novel method that used limit order book flow and history information for the determination of the stock movements using LSTM. The results of the proposed method were remarkably stationary. The authors of [154] used social media news, LDA features and RNN model to predict the trend of the index price. The authors of [218] proposed a novel method that used expert recommendations (Buy, Hold or Sell), emsemble of GRU and LSTM to predict the trend of the stocks prices.

CNN models with different data representations were also used for trend prediction. In [219], the authors used the last 100 entries from the limit order book to create images for the stock price prediction using CNN. Using the limit order book data to create 2D matrixlike format with CNN for predicting directional movement was innovative. In [159], HFT microstructures forecasting with CNN was implemented.

Table 13: Trend Forecasting Using Various Data

\begin{tabular}{|c|c|c|c|c|c|c|c|c|}
\hline Art. & Data Set & Period & Feature Set & Lag & Horizon & Method & $\begin{array}{l}\text { Performance } \\
\text { Criteria }\end{array}$ & Env. \\
\hline$[216]$ & $\begin{array}{lr}\text { Nasdaq } & \text { Nordic } \\
\text { (Kesko } & \text { Oyj, } \\
\text { Outokumpu } & \\
\text { Oyj, } & \text { Sampo, } \\
\text { Rautaruukki, Wart- } & \\
\text { sila Oyj) } & \\
\end{array}$ & 2010 & $\begin{array}{l}\text { Price and volume } \\
\text { data in LOB }\end{array}$ & $100 \mathrm{~s}$ & $\begin{array}{l}10 \mathrm{~s}, \\
20 \mathrm{~s}, \\
50 \mathrm{~s}\end{array}$ & LSTM & $\begin{array}{l}\text { Precision, Re- } \\
\text { call, F1-score, } \\
\text { Cohen's k }\end{array}$ & - \\
\hline$[217]$ & $\begin{array}{l}\text { High-frequency } \\
\text { record of all orders }\end{array}$ & $2014-2017$ & $\begin{array}{l}\text { Price } \\
\text { record of ata, } \\
\text { orders, transac- } \\
\text { tions }\end{array}$ & $2 \mathrm{~h}$ & - & LSTM & Accuracy & - \\
\hline$[154]$ & $\begin{array}{l}\text { Chinese, } \\
\text { Shanghai-Shenzhen } \\
300 \text { Stock Index } \\
\text { (HS300 }\end{array}$ & $2015-2017$ & $\begin{array}{lr}\begin{array}{l}\text { Social } \\
\text { news }\end{array} & \begin{array}{r}\text { media } \\
\text { (Sina }\end{array} \\
\text { Weibo), } & \text { price } \\
\text { data } & \\
\end{array}$ & $1 \mathrm{~d}$ & $1 \mathrm{~d}$ & $\begin{array}{l}\text { RNN-Boost } \\
\text { with LDA }\end{array}$ & $\begin{array}{l}\text { Accuracy, MAE, } \\
\text { MAPE, RMSE }\end{array}$ & $\begin{array}{l}\text { Python, } \\
\text { Scikit } \\
\text { learn }\end{array}$ \\
\hline$[218]$ & $\begin{array}{l}\text { ISMIS } 2017 \text { Data } \\
\text { Mining Competition } \\
\text { dataset }\end{array}$ & - & $\begin{array}{l}\text { Expert identifier, } \\
\text { class predicted by } \\
\text { expert }\end{array}$ & - & - & $\begin{array}{l}\text { LSTM } \\
\text { GRU } \\
\text { FCNN }\end{array}$ & Accuracy & - \\
\hline
\end{tabular}


Table 13: Trend Forecasting Using Various Data

\begin{tabular}{|c|c|c|c|c|c|c|c|c|}
\hline Art. & Data Set & Period & Feature Set & Lag & Horizon & Method & $\begin{array}{l}\text { Performance } \\
\text { Criteria }\end{array}$ & Env. \\
\hline$[219]$ & $\begin{array}{lr}\text { Nasdaq } & \text { Nordic } \\
\text { (Kesko } & \text { Oyj, } \\
\text { Outokumpu } & \\
\text { Oyj, } & \text { Sampo, } \\
\text { Rautaruukki, } & \text { Wart- } \\
\text { sila Oyj) } & \\
\end{array}$ & 2010 & $\begin{array}{l}\text { Price, } \quad \text { Volume } \\
\text { data, } 10 \text { orders } \\
\text { of the LOB }\end{array}$ & - & - & $\mathrm{CNN}$ & $\begin{array}{l}\text { Precision, Re- } \\
\text { call, F1-score, } \\
\text { Cohen's k }\end{array}$ & $\begin{array}{l}\text { Theano, } \\
\text { Scikit } \\
\text { learn, } \\
\text { Python }\end{array}$ \\
\hline [159] & $\begin{array}{l}\begin{array}{l}\text { London } \\
\text { change }\end{array} \\
\text { chock Ex- }\end{array}$ & $2007-2008$ & $\begin{array}{l}\text { Limit order book } \\
\text { state, trades, } \\
\text { buy/sell orders, } \\
\text { order deletions }\end{array}$ & - & - & $\mathrm{CNN}$ & Accuracy, kappa & Caffe \\
\hline
\end{tabular}

\section{Current Snaphot of The Field}

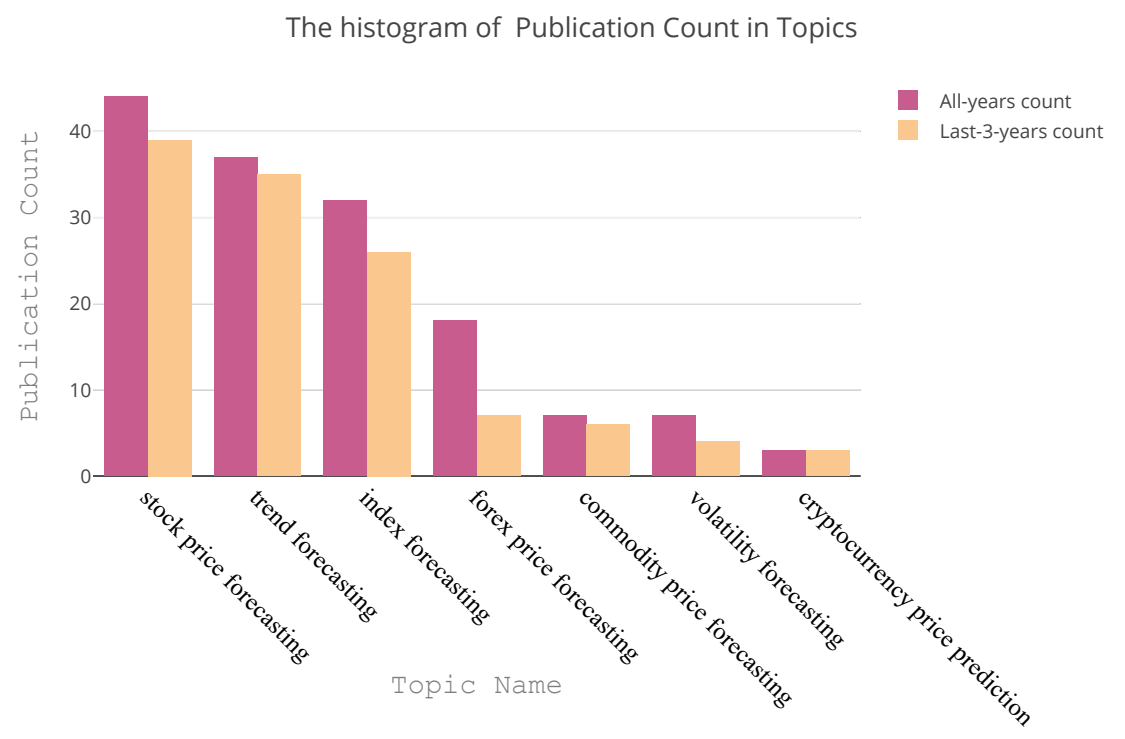

Figure 5: The histogram of Publication Count in Topics

After reviewing through all the research papers specifically targeted for financial time series forecasting implementations using DL models, we are now ready to provide some overall statistics about the current state of the studies. The number of papers that we were able to locate to be included in our survey was 140 . We categorized the papers according to their forecasted asset type. Furthermore, we also analyzed the studies through their DL model choices, frameworks for the development environment, data sets, comparable benchmarks, and some other differentiating criteria like feature sets, number of citations, etc. which we were not able to include in the paper due to space constraints. We will now summarize our notable observations to provide important highlights for the interested researchers within the field. 


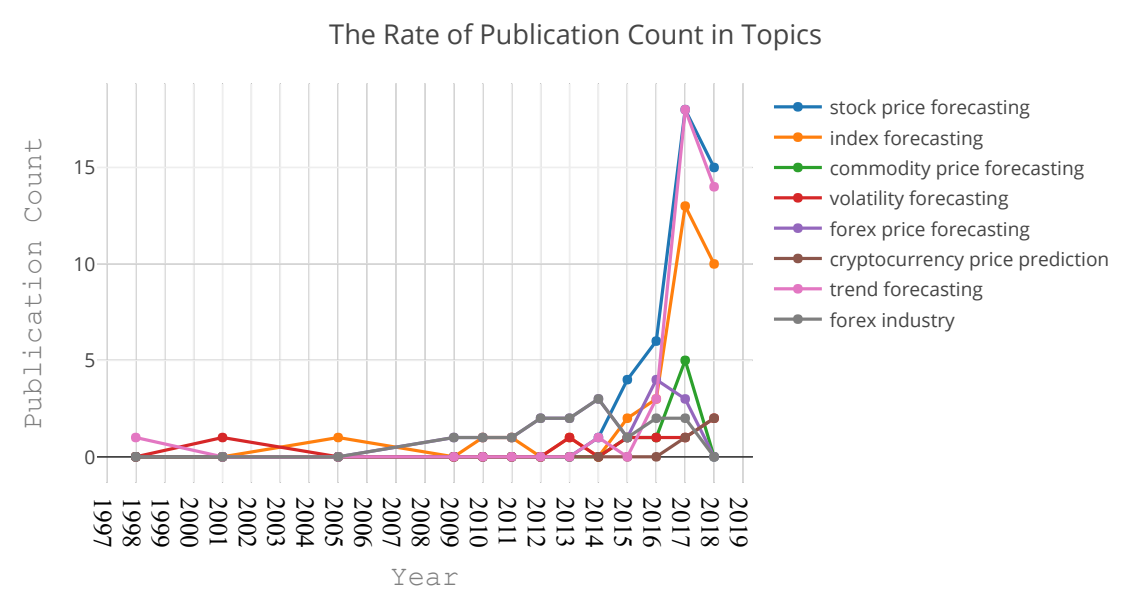

Figure 6: The rate of Publication Count in Topics

Figure 5 presents the various asset types that the researchers decided to develop their corresponding forecasting models for. As expected, stock market-related prediction studies dominate the field. Stock price forecasting, trend forecasting and index forecasting were the top three picks for the financial time series forecasting research. So far, 46 papers were published for stock price forecasting, 38 for trend forecasting and 33 for index forecasting, respectively. These studies constitute more than $70 \%$ of all studies indicating high interest. Following those include 19 papers for forex prediction and 7 papers for volatility forecasting. Meanwhile cryptocurrency forecasting has started attracting researchers, however, there were just 3 papers published yet, but this number is expected to increase in coming years [220]. Figure 6 highlights the rate of publication counts for various implementation areas throughout the years. Meanwhile Figure 7 provides more details about the choice of DL models over various implementation areas.

Figure 8 illustrates the accelerating appetite in the last 3 years by researchers for developing DL models for the financial time series implementations. Meanwhile, as Figure 9 indicates, most of the studies were published in journals (57 of them) and conferences (49 papers) even though a considerable amount of arXiv papers (11) and graduate theses (6) also exist.

One of the most important questions for a researcher is where he/she can publish their research findings. During our review of the papers, we also carefully investigated where each paper was published. We tabulated our results for the top journals for financial time series forecasting in Fig 10. According to these results, the journals with the most published papers include Expert Systems with Applications, Neurocomputing, Applied Soft Computing, The Journal of Supercomputing, Decision Support Systems, Knowledge-based Systems, 


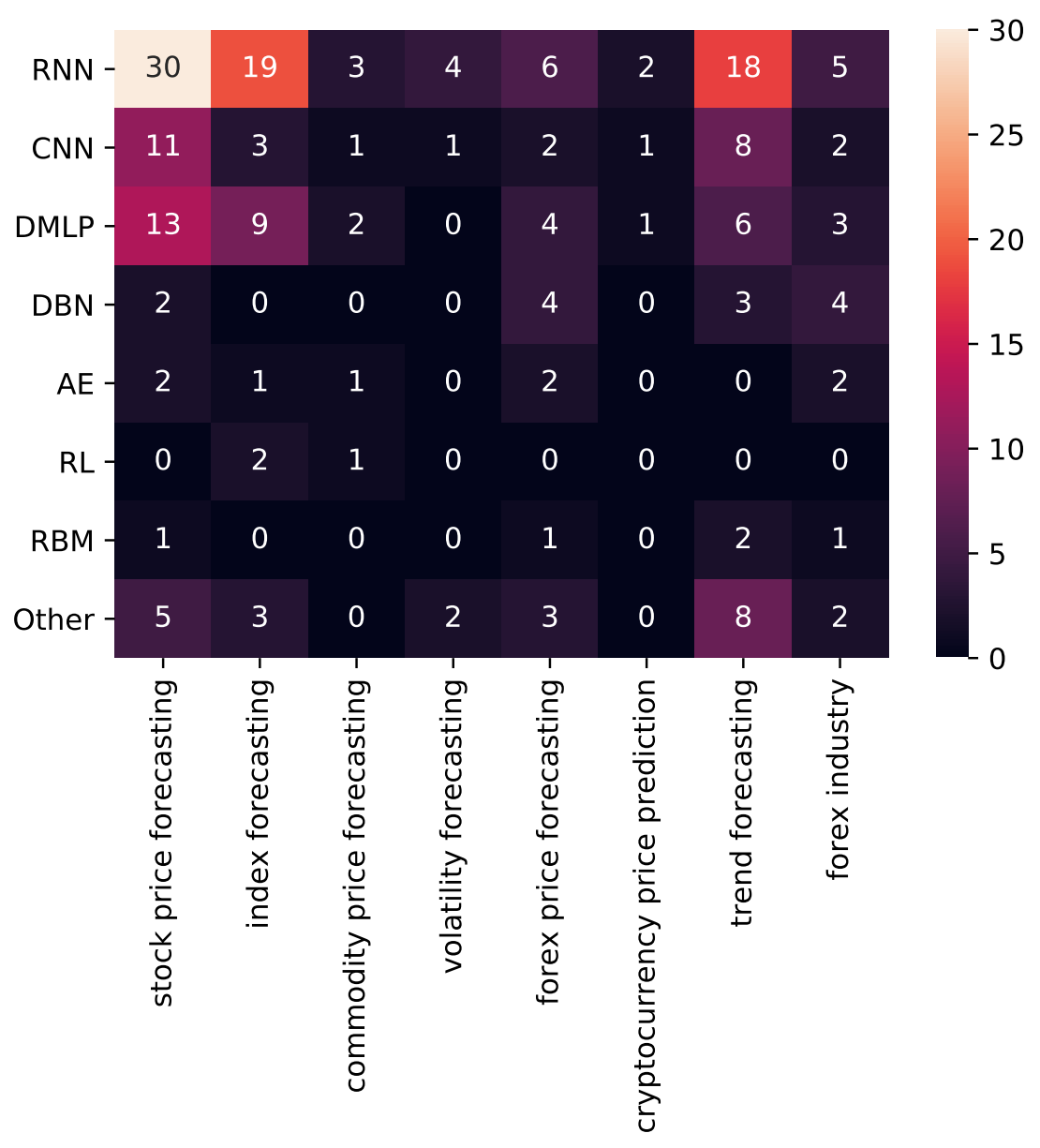

Figure 7: Topic-Model Heatmap

European Journal of Operational Research and IEEE Access. The interested researchers should also consider the trend within the last 3 years, as tendencies can be slightly varying depending on the particular implementation areas.

Carefully analyzing Figure 11 clearly validates the dominance of RNN based models (65 papers) among all others for DL model choices, followed by DMLP (23 papers) and CNN (20 papers). The inner-circle represents all years considered, meanwhile the outer circle just provides the studies within the last 3 years. We should note that RNN is a general model with several versions including LSTM, GRU, etc. Within RNN, the researchers mostly prefer LSTM due to its relative easiness of model development phase, however, other types of RNN are also common. Figure 12 provides a snapshot of the RNN model distribution. As mentioned above, LSTM had the highest interest among all with 58 papers, while Vanilla RNN and GRU had 27 and 10 papers respectively. Hence, it is clear that LSTM was the most popular DL model for financial time series forecasting or regression studies.

Meanwhile, DMLP and CNN generally were preferred for classification problems. Since the time series data generally consists of temporal components, some data preprocessing 


\section{Histogram of Publication Count in Years}



Figure 8: The histogram of Publication Count in Years

The histogram of Publication Count in Publication Type



Figure 9: The histogram of Publication Count in Publication Types

might be required before the actual classification can occur. Hence, a lot of these implementations utilize feature extraction, selection techniques along with possible dimensionality reduction methods. A lot of researchers decided to use DMLP mostly due to the fact that its shallow version MLP has been used extensively before and has a proven successful track record for many different financial applications including financial time series forecasting. 
The histogram of Top Journals

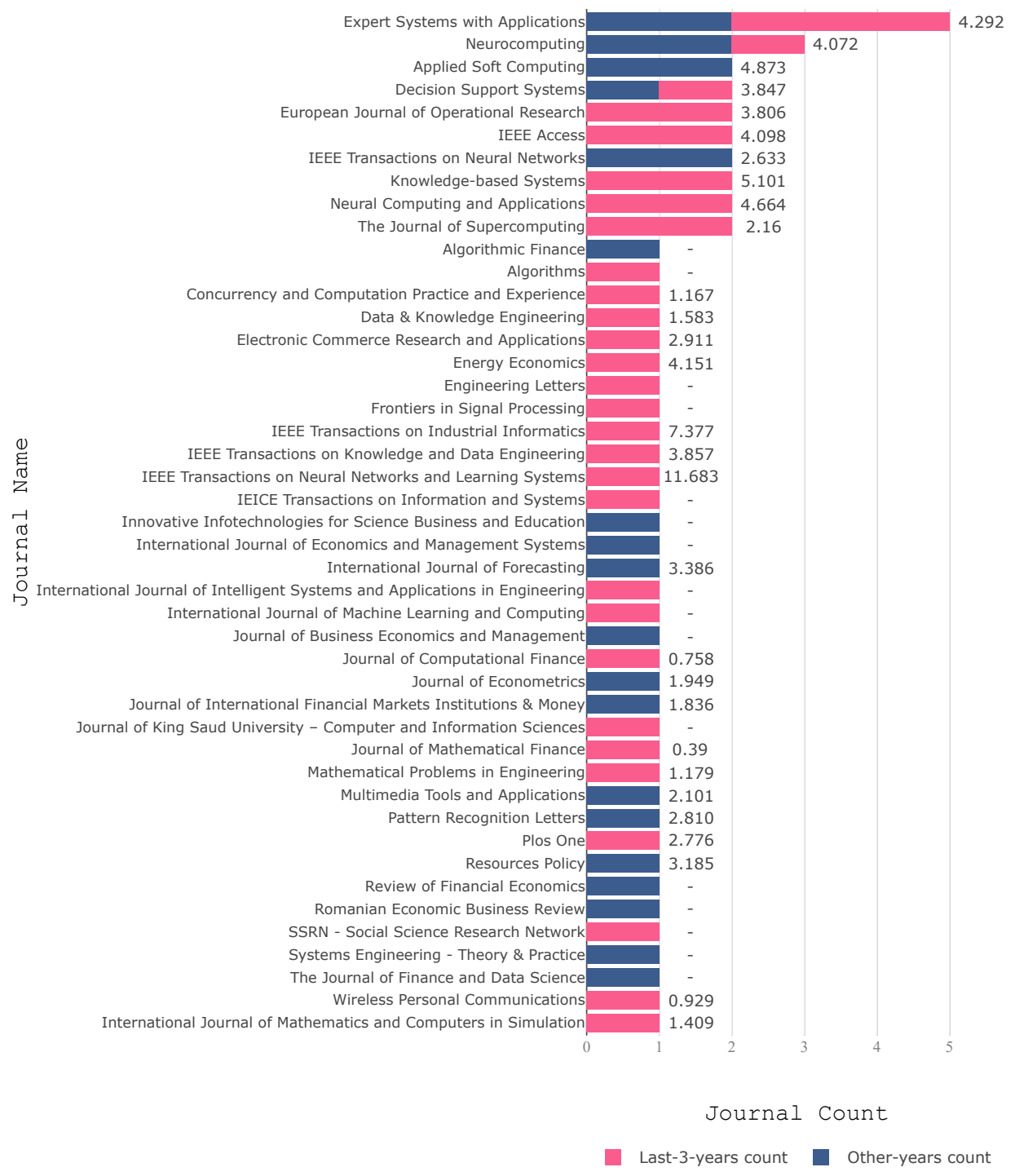

Figure 10: Top Journals - corresponding numbers next to the bar graph are representing the impact factor of the journals

Consistent with our observations, DMLP was also mostly preferred in the stock, index or in particular trend forecasting, since it is by definition, a classification problem with two (uptrend or downtrend) and three (uptrend, stationary or downtrend) class instances.

In addition to DMLP, CNN was also a popular choice for classification type financial time series forecasting implementations. Most of these studies appeared within the last 3 years. 


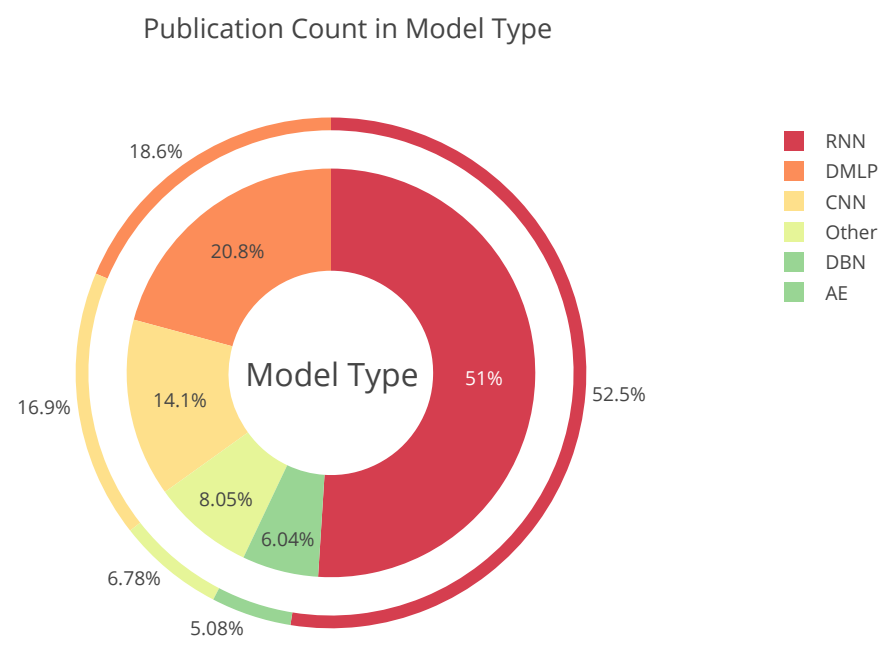

Figure 11: The Piechart of Publication Count in Model Types

Distribution of RNN Models

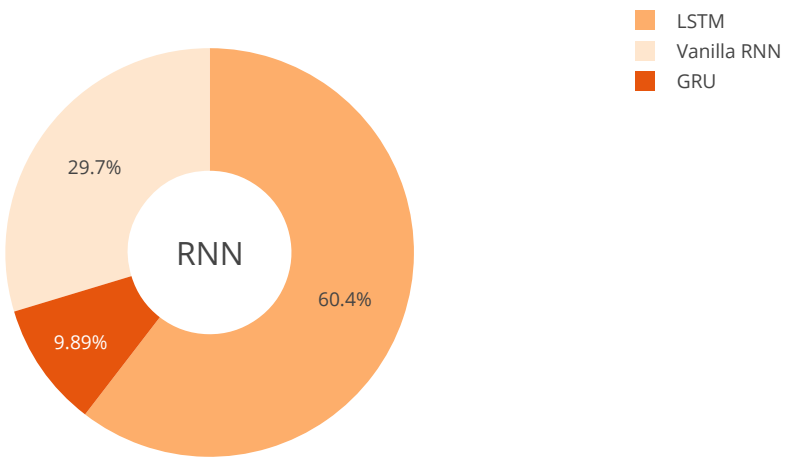

Figure 12: Distribution of RNN Models

As mentioned before, in order to convert the temporal time-varying sequential data into a more stationary classifiable form, some preprocessing might be necessary. Even though some 1-D representations exist, the 2-D implementation for CNN was more common, mostly inherited through image recognition applications of CNN from computer vision implementations. In some studies [188, 189, 193, 199, 219], innovative transformations of financial time 
series data into an image-like representation has been adapted and impressive performance results have been achieved. As a result, CNN might increase its share of interest for financial time series forecasting in the next few years.

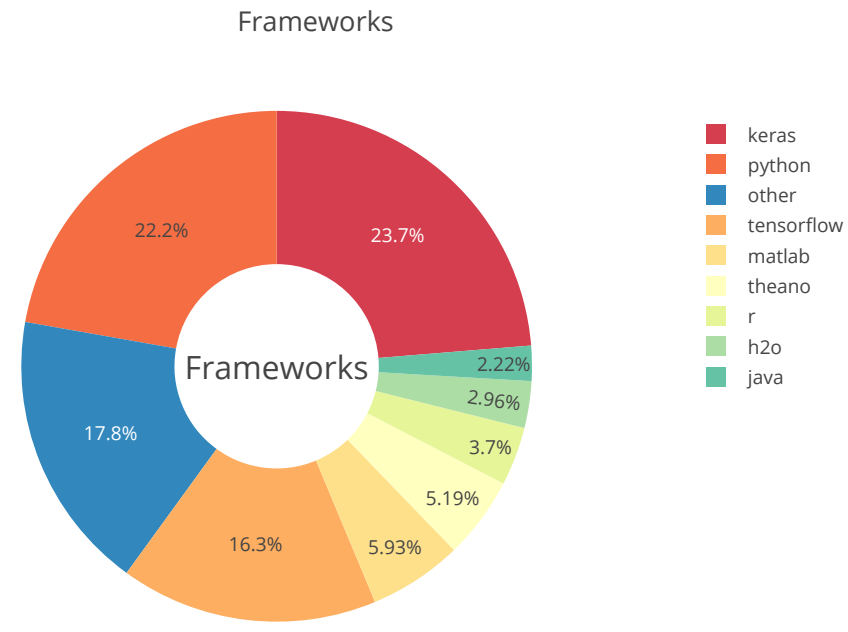

Figure 13: The Preferred Development Environments

As one final note, Figure 13 shows which frameworks and platforms the researchers and developers used while implementing their work. We tried to extract this information from the papers to the best of our effort. However, we need to keep in mind that not every publication provided their development environment. Also in most of the papers, generally, the details were not given preventing us from a more thorough comparison chart, i.e. some researchers claimed they used Python, but no further information was given, while some others mentioned the use of Keras or TensorFlow providing more details. Also, within the "Other" section the usage of Pytorch is on the rise in the last year or so, even though it is not visible from the chart. Regardless, Python-related tools were the most influential technologies behind the implementations covered in this survey.

\section{Discussion and Open Issues}

From an application perspective, even though financial time series forecasting has a relatively narrow focus, i.e. the implementations were mainly based on price or trend prediction, depending on the underlying DL model, very different and versatile models exist in literature. We need to keep in mind that, even though financial time series forecasting is a subset of time-series studies, due to the embedded profit-making expectations through successful prediction models, some differences exist, such that higher prediction accuracy sometimes might not reflect a profitable model. Hence, the risk and reward structure must also be 
taken into consideration. At this point, we will try to elaborate on our observations about these differences in various model designs and implementations.

\subsection{Models for financial time series forecasting}

According to the publication statistics, LSTM was the preferred choice of most researchers for financial time series forecasting. LSTM and its variations utilized the timevarying data with feedback embedded representations, resulting in higher performances for time series prediction implementations. Since most of the financial data, one way or another, included time-dependent components, LSTM was the natural choice in financial time series forecasting problems. Meanwhile, LSTM is a special DL model deriven from a more general classifier family, namely RNN.

Careful analysis of Figure 11 illustrates the dominance of RNN (which is highly consisted of LSTM). As a matter of fact, more than half of the published papers for time series forecasting studies fall into the RNN model category. Regardless of its problem type, price or trend prediction, the ordinal nature of the data representation forced the researchers to consider RNN, GRU and LSTM as viable preferences for their model choices. Hence, RNN models were chosen, at least for benchmarking, in a lot of studies for performance comparison against other developed models.

Meanwhile, other models were also used for time series forecasting problems. Among those, DMLP had the most interest due to the market dominance of its shallow cousin, MLP and its wide acceptance and long history within ML society. However, there is a fundamental difference in how DMLP and RNN based models were used for financial time series prediction problems.

DMLP fits well for both regression and classification problems. However, in general, data order independence must be preserved for better utilizing the internal working dynamics of such networks, even though through the learning algorithm configuration, some adjustments can be performed. In most cases, either trend components of the data need to be removed from the underlying time series, or some data transformations might be needed so that the resulting data becomes stationary. Regardless, some careful preprocessing might be necessary for the DMLP model to be successful. In contrast, RNN based models can directly work with time-varying data, making it easier for researchers to develop DL models.

As a result, most of the DMLP implementations had embedded data preprocessing before the learning stage. However, this inconvenience did not prevent the researchers to use DMLP and its variations during their model development process. Instead, a lot of versatile data representations were attempted in order to achieve higher overall prediction performances. A combination of fundamental and/or technical analysis parameters along with other features like financial sentiment through text mining was embedded into such models. In most of the DMLP studies, the corresponding problem was treated as classification, especially in trend prediction models, whereas RNN based models directly predicted the next value of the time series. Both approaches had some success in beating the underlying benchmark; hence it is not possible to claim victory of one model type over the other. However, for the general rule of thumb, researchers prefer RNN based models for time series regression and DMLP for trend classification (or buy-sell point identification) 
Another model that started becoming popular recently is CNN. CNN also works better for classification problems and unlike RNN based models, it is more suitable for either non-time varying or static data representations. The comments for DMLP are also mostly valid for CNN. Furthermore, unlike DMLP, CNN mostly requires locality within the data representation for better-performing classification results. One particular implementation area of CNN is image-based object recognition problems. In recent years, CNN based models dominated this field, handily outperforming all other models. Meanwhile, most financial data is time-varying and it might not be easy to implement CNN directly for financial applications. However, in some recent studies, various independent research groups followed an innovative transformation of 1-D time-varying financial data into 2-D mostly stationary image-like data so that they could utilize the power of CNN through adaptive filtering and implicit dimensionality reduction. Hence, with that approach, they were able to come up with successful models.

There is also a rising trend to use deep RL based financial algorithmic trading implementations; these are mostly associated with various agent-based models where different agents interact and learn from their interactions. This field even has more opportunities to offer with advancements in financial sentiment analysis through text mining to capture investor psychology; as a result, behavioral finance can benefit from these particular studies associated with RL based learning models coupled with agent-based studies.

Other models including DBN, AE and RBM also were used by several researchers and superior performances were reported in some of their work; but the interested readers need to check these studies case by case to see how they were modelled both from the data representation and learning point of view.

\subsection{Discussions on Selected Features}

Regardless of the underlying forecasting problem, somehow the raw time series data was almost always embedded directly or indirectly within the feature vector, which is particularly valid for RNN-based models. However, in most of the other model types, other features were also included. Fundamental analysis and technical analysis features were among the most favorable choices for stock/index forecasting studies.

Meanwhile, in recent years, financial text mining is particularly getting more attention, mostly for extracting the investor/trader sentiment. The streaming flow of financial news, tweets, statements, blogs allowed the researchers to build better and more versatile prediction and evaluation models integrating numerical and textual data. The general methodology involves in extracting financial sentiment analysis through text mining and combining that information with fundamental/technical analysis data to achieve better overall performance. It is logical to assume that this trend will continue with the integration of more advanced text and NLP techniques.

\subsection{Discussions on Forecasted Asset Types}

Even though forex price forecasting is always popular among the researchers and practitioners, stock/index forecasting has always had the most interest among all asset groups. 
Regardless, price/trend prediction and algo-trading models were mostly embedded with these prediction studies.

These days, one other hot area to financial time series forecasting research is involved with cryptocurrencies. Cryptocurrency price prediction has an increasing demand from the financial community. Since the topic is fairly new, we might see more studies and implementations coming in due to high expectations and promising rewards.

There were also a number of publications in commodity price forecasting research, in particular, the price of oil. Oil price prediction is crucial due to its tremendous effect on world economic activities and planning. Meanwhile, gold is considered a safe investment and almost every investor, at one time, considers allocating some portion of their portfolios for gold-related investments. In times of political uncertainties, a lot of people turn to gold for protecting their savings. Even though we have not encountered a noteworthy study for gold price forecasting, due to its historical importance, there might be opportunities in this area for the years to come.

\subsection{Open Issues and Future Work}

Despite the general motivation for financial time series forecasting remaining fairly unchanged, the means of achieving the financial goals vary depending on the choices and trade-off between the traditional techniques and newly developed models. Since our fundamental focus is on the application of DL for financial time series studies, we will try to asses the current state of the research and extrapolate that into the future.

\subsubsection{Model Choices for the Future}

The dominance of RNN-based models for price/trend prediction will probably not disappear anytime soon, mainly due to their easy adaptation to most asset forecasting problems. Meanwhile, some enhanced versions of the original LSTM or RNN models, generally integrated with hybrid learning systems started becoming more common. Readers need to check individual studies and assess their performances to see which one fits the best for their particular needs and domain requirements.

We have observed the increasing interest in 2-D CNN implementations of financial forecasting problems through converting the time series into an image-like data type. This innovative methodology seems to work quite satisfactorily and provides promising opportunities. More studies of this kind will probably continue in the near future.

Nowadays, new models are generated through older models via modifying or enhancing the existing models so that better performances can be achieved. Such topologies include Generative Adversarial Network (GAN), Capsule networks, etc. They have been used in various non-financial studies, however, financial time series forecasting has not been investigated for those models yet. As such, there can be exciting opportunities both from research and practical point of view.

Another DL model that is not investigated thoroughly is Graph CNN. Graphs can be used to represent portfolios, social networks of financial communities, fundamental analysis data, etc. Even though graph algorithms can directly be applied to such configurations, different graph representations can also be implemented for the time series forecasting problems. Not 
much has been done on this particular topic, however, through graph representations of the time series data and implementing graph analysis algorithms, or implementing CNN through these graphs are among the possibilities that the researchers can choose.

As a final note for the future models, we believe deep RL and agent-based models offer great opportunities for the researchers. HFT algorithms, robo-advisory systems highly depend on automated algorithmic trading systems that can decide what to buy and when to buy without any human intervention. These aforementioned models can fit very well in such challenging environments. The rise of the machines will also lead to a technological (and algorithmic) arms race between Fintech companies and quant funds to be the best in their neverending search for "achieving alpha". New research in these areas can be just what the doctor ordered.

\subsubsection{Future Projections for Financial Time Series Forecasting}

Most probably, for the foreseeable future, the financial time series forecasting will have a close research cooperation with the other financial application areas like algorithmic trading and portfolio management, as it was the case before. However, changes in the available data characteristics and introduction of new asset classes might not only alter the forecasting strategies of the developers, but also force the developers to look for new or alternative techniques to better adapt to these new challenging working conditions. In addition, metrics like Continuous Ranked Probability Score (CRPS) for evaluating probability distributions might be included for more thorough analysis.

One rising trend, not only for financial time series forecasting, but for all intelligent decision support systems, is the human-computer interaction and NLP research. Within that field, text mining and financial sentiment analysis areas are of particular importance to financial time series forecasting. Behavioral finance may benefit from the new advancements in these fields.

In order to utilize the power of text mining, researchers started developing new data representations like Stock2Vec [204] that can be useful for combining textual and numerical data for better prediction models. Furthermore, NLP based ensemble models that integrate data semantics with time-series data might increase the accuracy of the existing models.

One area that can benefit a lot from the interconnected financial markets is the automated statistical arbitrage trading model development. It has been used in forex and commodity markets before. In addition, a lot of practitioners currently seek arbitrage opportunities in the cryptocurrency markets [220], due to the existence of the huge number of coins available on various marketplaces. Price disruptions, high volatility, bid-ask spread variations cause arbitrage opportunities across different platforms. Some opportunists develop software models that can track these price anomalies for the instant materialization of profits. Also, it is possible to construct pairs trading portfolios across different asset classes using appropriate models. It is possible that DL models can learn (or predict) these opportunities faster and more efficient than classical rule-based systems. This will also benefit HFT studies that are constantly looking for faster and more efficient trading algorithms and embedded systems with minimum latency. In order to achieve that, Graphics Processing Unit (GPU) or Field Programmable Gate Array (FPGA) based hardware solutions embedded with DL models 
can be utilized. There is a lack of research accomplished on this hardware aspect of financial time series forecasting and algorithmic trading. As long as there is enough computing power available, it is worth investigating the possibilities for better algorithms, since the rewards are high.

\subsection{Responses to our Initial Research Questions}

We are now ready to go back to our initially stated research questions. Our question and answer pairs, through our observations, are as follows:

- Which DL models are used for financial time series forecasting ?

Response: RNN based models (in particular LSTM) are the most commonly used models. Meanwhile, CNN and DMLP have been used extensively in classification type implementations (like trend classification) as long as appropriate data processing is applied to the raw data.

- How is the performance of DL models compared with traditional machine learning counterparts?

Response: In the majority of the studies, DL models were better than ML. However, there were also many cases where their performances were comparable. There were even two particular studies $([82,175]$ where ML models performed better than DL models. Meanwhile, appetite for preferrance of DL implementations over ML models is growing. Advances in computing power, availability of big data, superior performance, implicit feature learning capabilities and user friendly model development environment for DL models are among the main reasons for this migration.

- What is the future direction for DL research for financial time series forecasting ?

Response: NLP, semantics and text mining-based hybrid models ensembled with timeseries data might be more common in the near future.

\section{Conclusions}

Financial time series forecasting has been very popular among ML researchers for more than 40 years. The financial community got a new boost lately with the introduction of DL implementations for financial prediction research and a lot of new publications appeared accordingly. In our survey, we wanted to review the existing studies to provide a snapshot of the current research status of DL implementations for financial time series forecasting. We grouped the studies according to their intended asset class along with the preferred DL model associated with the problem. Our findings indicate, even though financial forecasting has a long research history, overall interest within the DL community is on the rise through utilizing new DL models; hence, a lot of opportunities exist for researchers. 


\section{Acknowledgement}

This work is supported by Scientific and Technological Research Council of Turkey (TUBITAK) grant no 215E248.

\section{Glossary}

AdaGrad Adaptive Gradient Algorithm. 6, 7

ADAM Adaptive Moment Estimation. 6-8, 13, 14,33

AE Autoencoder. 5, 9, 13, 14, 18, 19, 26, 29-31, 46

AI Artificial Intelligence. 3

AIS Annealed Importance Sampling. 12

AMEX American Stock Exchange. 20, 21

ANN Artificial Neural Network. 3-5, 8, 11-13, 18, $20,23,24,31,33,34$

AR Active Return. 21

AR Autoregressive. 22

ARCH Autoregressive Conditional Heteroscedasticity. 32

ARIMA Autoregressive Integrated Moving Average. 19, 20,30, 32

ARMA Autoregressive Moving Average. 28, 32

AUC Area Under the Curve. 21

AUROC Area Under the Receiver Operating Characteristics. 33, 34, 36, 37

B\&H Buy and Hold. 25

BELM Basic Extreme Learning Machine. 37

Bi-GRU Bidirectional Gated Recurrent Unit. 35, 36

Bi-LSTM Bidirectional LSTM. 22, 24, 25

BIST Istanbul Stock Exchange Index. 20, 21, 23, 24

Bovespa Brazilian Stock Exchange. 23, 25

BPTT Back Propagation Through Time. 7, 8

BSE Bombay Stock Exchange. 26

CCI Commodity Channel Index. 27

CD Contrastive Divergence. 12, 13

CDAX German Stock Market Index Calculated by Deutsche Börse. 21

CDBN Continuous-valued Deep Belief Networks. 30

CDBN-FG Fuzzy Granulation with Continuousvalued Deep Belief Networks. 31

CNN Convolutional Neural Network. 1, 3, 5, 6, 10, 17-22, 25-38, 40, 42-44, 46-49

CRBM Continuous Restricted Boltzman machine. 30

CRPS Continuous Ranked Probability Score. 48
CSE Colombo Stock Exchange. 18

CSI China Securities Index. 18, 22, 23, 26, 28, 29

DA Direction Accuracy. 31

DAX The Deutscher Aktienindex. 29, 33

DBN Deep Belief Network. 1, 5, 12, 13, 18, 19, 28, $30,31,33-36,46$

DE Differential Evolution. 26

DFNN Deep Feedforward Neural Network. 17, 19, $22,23,29,33$

DGM Deep Neural Generative Model. 36, 37

DJI Dow Jones Index. 36

DJIA Dow Jones Industrial Average. 23-26, 36

DL Deep Learning. 1-5, 7, 9, 10, 13, 14, 16-21, 23, $25,27,29,30,35,36,38-40,44,45,47-49$

DLR Deep Learning Representation. 37

DMLP Deep Multilayer Perceptron. 5-8, 10, 23, 30, 34, 35, 40-42, 45, 46, 49

DNN Deep Neural Network. 6, 10, 18-21, 23-28, $30-33,35,36$

DOW30 Dow Jones Industrial Average 30. 23, 2628

DP Dynamic Programming. 15, 16

DPA Direction Prediction Accuracy. 21

DRL Deep Reinforcement Learning. 3, 5, 16

DRSE Deep Random Subspace Ensembles. 36

DWNN Deep and Wide Neural Network. 18, 19

EA Evolutionary Algorithm. 25, 27

EC Evolutionary Computation. 3, 4

EGARCH Exponential GARCH. 24

ELM Extreme Learning Machine. 18, 19, 37

EMA Exponential Moving Average. 27

EMD2FNN Empirical Mode Decomposition and Factorization Machine based Neural Network. 33,34

ETF Exchange-Traded Fund. 28, 31, 33, 34

FCNN Fully Connected Neural Network. 37

FDDR Fuzzy Deep Direct Reinforcement Learning. 23-25, 27, 28

FFNN Feedforward Neural Network. 13, 14, 20, $24,30,33$

FHSO Firefly Harmony Search Optimization. 25, 31

FLANN Functional Link Neural network. 27 
FNN Fully Connected Neural Network. 7, 27, 33

FTSE London Financial Times Stock Exchange Index. 23, 26, 28, 29, 31, 34

GA Genetic Algorithm. 4, 25, 34

GAF Gramian Angular Field. 33

GAN Generative Adversarial Network. 21, 47

GAN-FD GAN for minimizing Forecast error loss and Direction prediction loss. 20

GARCH Generalised Auto-Regressive Conditional Heteroscedasticity. 28, 29, 31, 32

GASVR GA with a SVR. 25, 26, 29

GBT Gradient Boosted Trees. 18, 19

GDAX Global Digital Asset Exchange. 26

GLM Generalized Linear Model. 24

GML Generalized Linear Model. 25

GP Genetic Programming. 3, 4, 30

GPA The Gaussian Process Approach. 7, 9

GRU Gated-Recurrent Unit. 9, 18, 19, 21, 22, 32$37,40,45$

GS Grid Search. 7, 9, 10, 12-14, 16

GSPC S\&P500 Commodity Price Index. 24

HAN Hybrid Attention Network. 36

HAR Heterogeneous Autoregressive Process. 25, 29

HAR-GASVR HAR with a GASVR. 24, 29

HFT High Frequency Trading. 18, 28, 37, 48

HIT Hit Rate. 23, 24

HMAE Heteroscedasticity Adjusted MAE. 29

HMM Hidden Markov Model. 35

HMRPSO Modified Version of PSO. 26

HMSE Heteroscedasticity Adjusted MSE. 29

HR Hit Rate. 21, 29, 31

HS China Shanghai Shenzhen Stock Index. 26, 27, 37

HSI Hong Kong Hang Seng Index. 23, 24, 26, 33

IBOVESPA Indice Bolsa de Valores de Sao Paulo. 34

IC Information Coeffiencient. 21

IR Information Ratio. 21

ISE Istanbul Stock Exchange Index. 24, 31

IXIC NASDAQ Composite Index. 24

KELM Kernel Extreme Learning Machine. 37

KL-Divergence Kullback Leibler Divergence. 12

KOSPI The Korea Composite Stock Price Index. $18,19,23-26,29$

KSE Korea Stock Exchange. 34

LAR Linear Auto-regression Predictor. 22

LDA Latent Dirichlet Allocation. 27, 37

LOB Limit Order Book Data. 37, 38

LRNFIS Locally Recurrent Neuro-fuzzy Information System. 23, 25, 31
LSTM Long-Short Term Memory. 1, 3, 5, 8, 9, 17-30, 32-37, 40, 45, 47, 49

MACD Moving Average Convergence and Divergence. 34,36

MAD Mean Absolute Deviation. 18

MAE Mean Absolute Error. 18, 21, 23, 24, 26, 27, $29,31,33,37$

MAM Moving Average Mapping. 33

MAPE Mean Absolute Percentage Error. 18, 2124, 26-29, 31, 33, 37

MASE Mean Standard Deviation. 23

MC Monte Carlo. 15, 16

MCC Matthew Correlation Coefficient. 22, 26, 36, 37

MDA Multilinear Discriminant Analysis. 20, 21

MDD Maximum Drawdown. 18

MDP Markov Decision Process. 14, 15

MI Mutual Information. 18

ML Machine Learning. 1-5, 7, 19, 25, 29, 30, 34, $35,45,49$

MLP Multilayer Perceptron. 10, 18-21, 23, 24, 30, $31,34,41,45$

MM Markov Model. 29

MODRL Multi-objective Deep Reinforcement Learning. 26

MoE Mixture of Experts. 24

MOEA Multiobjective Evolutionary Algorithm. 4

MRS Markov Regime Switching. 27

MS Manual Search. 7, 9, 10, 12-14, 16

MSE Mean Squared Error. 13, 14, 18, 20, 21, 24, 26, 28, 29, 31-34

MSFE Mean Squared Forecast Error. 24

MSPE Mean Squared Prediction Error. 20

NASDAQ National Association of Securities Dealers Automated Quotations. 18-21, 23, 26$28,33,34,36$

NIFTY National Stock Exchange of India. 23, 26, 36

NIKKEI Tokyo Nikkei Index. 23, 25, 26, 31

NLP Natural Language Processing. 9, 46, 48, 49

NMAE Normalized Mean Absolute Error. 29, 31

NMSE Normalized Mean Square Error. 18, 29, 31

NN Neural Network. 5, 6, 10, 16, 29, 33, 35-37

norm-RMSE Normalized RMSE. 18

NSE National Stock Exchange of India. 18

NYMEX New York Mercantile Exchange. 27, 28

NYSE New York Stock Exchange. 18, 20, 21, 23, 34,36

OCHL Open,Close,High, Low. 21, 24, 26

OCHLV Open,Close,High, Low, Volume. 18, 19, $21-24,26,32-34,36$ 
OMX Stockholm Stock Exchange. 23-26, 28, 31, 33,34

PCA Principal Component Analysis. 20, 21, 29, 37

PCC Pearson's Correlation Coefficient. 31

PCD Percentage of Correct Direction. 21

PLR Piecewise Linear Representation. 21

PNN Probabilistic Neural Network. 33

PPOSC Percentage Price Oscillator. 27

PSN Psi-Sigma Network. 30, 31

PSO Particle Swarm Optimization. 26, 27

$\mathbf{R}^{2}$ Squared correlation, Non-linear regression multiple correlation. 21

RBF Radial Basis Function Neural Network. 18, $19,24,31,34$

RBM Restricted Boltzmann Machine. 5, 11-13, 19, 30, 31, 34-36, 46

RCEFLANN Recurrent Computationally Efficient Functional Link Neural Network. 26, 27

RCNN Recurrent CNN. 22

ReLU Rectified Linear Unit. 5, 7, 12

RF Random Forest. 18, 19, 22, 29

RHE Recurrent Hybrid Elman. 24, 25, 31

RL Reinforcement learning. 14, 15, 23-25, 28, 46, 48

RMDN Recurrent Mixture Density Network. 29, 31

RMDN-GARCH RMDN with a GARCH. 29

RMSE Root Mean Square Error. 18, 21-24, 26-29, $31-33,37$

RMSProp Root Mean Square Propagation. 6-8, $10,13,14$

RMSRE Root Mean Square Relative Error. 21

RNN Recurrent Neural Network. 2, 5, 7-9, 18-33, 35-37, 40, 45-47, 49

RS RandomSearch. 7, 9, 10, 12-14, 16

RSE Relative Squared Error. 21

RSI Relative Strength Index. 27, 34

RW Random Walk. 27, 28, 30

S\&P500 Standard's \& Poor's 500 Index. 18-29, $31,33,36,37$

SCI SSE Composite Index. 22

SDAE Stacked Denoising Autoencoders. 27, 28

SFM State Frequency Memory. 18, 19
SGD Stochastic Gradient Descent. 6-8, 10, 13, 14

SLP Single Layer Perceptron. 34

SMAPE Symmetric Mean Absolute Percentage Error. 21

SMBGO Sequential Model-Based Global Optimization. 7, 9

SPY SPDR S\&P 500 ETF. 33

SR Sharpe-ratio. 21, 23-26, 28, 31

SRNN Stacked Recurrent Neural Network. 18, 19

SSE Shanghai Stock Exchange. 18, 21, 23, 26, 34

SSEC Shanghai Stock Exchange Composite. 33

STD Standard Deviation. 23, 25, 26

SVM Support Vector Machine. 20, 22, 33-36

SVR Support Vector Regressor. 25, 27, 30, 31

SZSE Shenzhen Stock Exchange Composite Index. 23-26, 28, 33

TAIEX Taiwan Capitalization Weighted Stock Index. 23, 26

TALIB Technical Analysis Library Package. 26, 34

TAQ Trade and Quote. 21

TAR Threshold Autoregressive. 24, 25, 31

TD Temporal Difference. 15, 16

TDNN Timedelay Neural Network. 33

TF-IDF Term Frequency-Inverse Document Frequency. 37

TGRU Two-stream GRU. 35

THEIL-U Theil's inequality coefficient. 26, 31

TR Total Return. 21, 24

TSPEA Tree-structured Parzen Estimator Approach. 7,9

TUNINDEX Tunisian Stock Market Index. 24

TWSE Taiwan Stock Exchange. 22, 26, 27

VEC Vector Error Correction model. 24, 25, 31

VIX S\&P500 Volatility Index. 23-25, 29

VXD Dow Jones Industrial Average Volatility Index. 24, 25, 29

VXN NASDAQ100 Volatility Index. 23-25, 29

WHR Weighted Hit Rate. 29, 31

William\%R Williams Percent Range. 27

WMTR Weighted Multichannel Time-series Regression. 20, 21

WT Wavelet Transforms. 26

WTI West Texas Intermediate. 28

XGBoost eXtreme Gradient Boosting. 34, 35 


\section{References}

[1] Ahmet Murat Ozbayoglu, Mehmet Ugur Gudelek, and Omer Berat Sezer. Deep learning for financial applications: A survey. working paper, 2019.

[2] Rafik A. Aliev, Bijan Fazlollahi, and Rashad R. Aliev. Soft computing and its applications in business and economics. In Studies in Fuzziness and Soft Computing, 2004.

[3] Ludmila Dymowa. Soft Computing in Economics and Finance. Springer Berlin Heidelberg, 2011.

[4] Boris Kovalerchuk and Evgenii Vityaev. Data Mining in Finance: Advances in Relational and Hybrid Methods. Kluwer Academic Publishers, Norwell, MA, USA, 2000.

[5] Anthony Brabazon and Michael O'Neill, editors. Natural Computing in Computational Finance. Springer Berlin Heidelberg, 2008.

[6] Arash Bahrammirzaee. A comparative survey of artificial intelligence applications in finance: artificial neural networks, expert system and hybrid intelligent systems. Neural Computing and Applications, 19(8):1165-1195, June 2010.

[7] D. Zhang and L. Zhou. Discovering golden nuggets: Data mining in financial application. IEEE Transactions on Systems, Man and Cybernetics, Part C (Applications and Reviews), 34(4):513-522, November 2004.

[8] Asunción Mochón, David Quintana, Yago Sáez, and Pedro Isasi Viñuela. Soft computing techniques applied to finance. Applied Intelligence, 29:111-115, 2007.

[9] Sendhil Mullainathan and Jann Spiess. Machine learning: An applied econometric approach. Journal of Economic Perspectives, 31(2):87-106, May 2017.

[10] Shu-Heng Chen, editor. Genetic Algorithms and Genetic Programming in Computational Finance. Springer US, 2002.

[11] Ma. Guadalupe Castillo Tapia and Carlos A. Coello Coello. Applications of multi-objective evolutionary algorithms in economics and finance: A survey. In 2007 IEEE Congress on Evolutionary Computation. IEEE, September 2007.

[12] Antonin Ponsich, Antonio Lopez Jaimes, and Carlos A. Coello Coello. A survey on multiobjective evolutionary algorithms for the solution of the portfolio optimization problem and other finance and economics applications. IEEE Transactions on Evolutionary Computation, 17(3):321-344, June 2013.

[13] Ruben Aguilar-Rivera, Manuel Valenzuela-Rendon, and J.J. Rodriguez-Ortiz. Genetic algorithms and darwinian approaches in financial applications: A survey. Expert Systems with Applications, 42(21): 7684-7697, November 2015.

[14] Roy Rada. Expert systems and evolutionary computing for financial investing: A review. Expert Systems with Applications, 34(4):2232-2240, 2008.

[15] Yuhong Li and Weihua Ma. Applications of artificial neural networks in financial economics: A survey. In 2010 International Symposium on Computational Intelligence and Design. IEEE, October 2010.

[16] Michal Tkáč and Robert Verner. Artificial neural networks in business: Two decades of research. Applied Soft Computing, 38:788 - 804, 2016.

[17] B. Elmsili and B. Outtaj. Artificial neural networks applications in economics and management research: An exploratory literature review. In 2018 4th International Conference on Optimization and Applications (ICOA), pages 1-6, April 2018.

[18] Marc-André Mittermayer and Gerhard F Knolmayer. Text mining systems for market response to news: A survey. September 2006.

[19] Leela Mitra and Gautam Mitra. Applications of news analytics in finance: A review. In The Handbook of News Analytics in Finance, pages 1-39. John Wiley \& Sons, Ltd., May 2012.

[20] Arman Khadjeh Nassirtoussi, Saeed Aghabozorgi, Teh Ying Wah, and David Chek Ling Ngo. Text mining for market prediction: A systematic review. Expert Systems with Applications, 41(16):76537670, November 2014.

[21] Colm Kearney and Sha Liu. Textual sentiment in finance: A survey of methods and models. International Review of Financial Analysis, 33:171-185, May 2014.

[22] B. Shravan Kumar and Vadlamani Ravi. A survey of the applications of text mining in financial domain. Knowledge-Based Systems, 114:128-147, December 2016. 
[23] Frank Z. Xing, Erik Cambria, and Roy E. Welsch. Natural language based financial forecasting: a survey. Artificial Intelligence Review, 50(1):49-73, October 2017.

[24] Bruce J Vanstone and Clarence Tan. A survey of the application of soft computing to investment and financial trading. In Brian C Lovell, Duncan A Campbell, Clinton B Fookes, and Anthony J Maeder, editors, Proceedings of the Eighth Australian and New Zealand Intelligent Information Systems Conference (ANZIIS 2003), pages 211-216. The Australian Pattern Recognition Society, 2003. Copyright The Australian Pattern Recognition Society 2003. All rights reserved. Permission granted.

[25] Ehsan Hajizadeh, H. Davari Ardakani, and Jamal Shahrabi. Application of data mining techniques in stock markets : A survey. 2010.

[26] Binoy B. Nair and V.P. Mohandas. Artificial intelligence applications in financial forecasting - a survey and some empirical results. Intelligent Decision Technologies, 9(2):99-140, December 2014.

[27] Rodolfo C. Cavalcante, Rodrigo C. Brasileiro, Victor L.F. Souza, Jarley P. Nobrega, and Adriano L.I. Oliveira. Computational intelligence and financial markets: A survey and future directions. Expert Systems with Applications, 55:194-211, August 2016.

[28] Bjoern Krollner, Bruce J. Vanstone, and Gavin R. Finnie. Financial time series forecasting with machine learning techniques: a survey. In ESANN, 2010.

[29] P. D. Yoo, M. H. Kim, and T. Jan. Machine learning techniques and use of event information for stock market prediction: A survey and evaluation. In International Conference on Computational Intelligence for Modelling, Control and Automation and International Conference on Intelligent Agents, Web Technologies and Internet Commerce (CIMCA-IAWTIC'06), volume 2, pages 835-841, November 2005.

[30] G Preethi and B Santhi. Stock market forecasting techniques: A survey. Journal of Theoretical and Applied Information Technology, 46:24-30, December 2012.

[31] George S. Atsalakis and Kimon P. Valavanis. Surveying stock market forecasting techniques - part ii: Soft computing methods. Expert Systems with Applications, 36(3):5932-5941, April 2009.

[32] Amitava Chatterjee, O.Felix Ayadi, and Bryan E. Boone. Artificial neural network and the financial markets: A survey. Managerial Finance, 26(12):32-45, December 2000.

[33] R. Katarya and A. Mahajan. A survey of neural network techniques in market trend analysis. In 2017 International Conference on Intelligent Sustainable Systems (ICISS), pages 873-877, December 2017.

[34] Yong Hu, Kang Liu, Xiangzhou Zhang, Lijun Su, E.W.T. Ngai, and Mei Liu. Application of evolutionary computation for rule discovery in stock algorithmic trading: A literature review. Applied Soft Computing, 36:534-551, November 2015.

[35] Wei Huang, K. K. Lai, Y. Nakamori, and Shouyang Wang. Forecasting foreign exchange rates with artificial neural networks: A review. International Journal of Information Technology 85 Decision Making, 03(01):145-165, 2004.

[36] Dadabada Pradeepkumar and Vadlamani Ravi. Soft computing hybrids for forex rate prediction: A comprehensive review. Computers \&3 Operations Research, 99:262 - 284, 2018.

[37] Yann LeCun, Yoshua Bengio, and Geoffrey Hinton. Deep learning. Nature, 521(7553):436-444, 2015.

[38] Jürgen Schmidhuber. Deep learning in neural networks: An overview. Neural networks, 61:85-117, 2015.

[39] Ian Goodfellow, Yoshua Bengio, and Aaron Courville. Deep Learning. MIT Press, 2016. http://www.deeplearningbook.org.

[40] George Cybenko. Approximation by superpositions of a sigmoidal function. Mathematics of control, signals and systems, 2(4):303-314, 1989.

[41] Barry L Kalman and Stan C Kwasny. Why tanh: choosing a sigmoidal function. In [Proceedings 1992] IJCNN International Joint Conference on Neural Networks, volume 4, pages 578-581. IEEE, 1992.

[42] Vinod Nair and Geoffrey E Hinton. Rectified linear units improve restricted boltzmann machines. In Proceedings of the 27th international conference on machine learning (ICML-10), pages 807-814, 2010.

[43] Andrew L Maas, Awni Y Hannun, and Andrew Y Ng. Rectifier nonlinearities improve neural network acoustic models. In Proc. icml, volume 30, page 3, 2013. 
[44] Prajit Ramachandran, Barret Zoph, and Quoc V Le. Searching for activation functions. arXiv preprint arXiv:1710.05941, 2017.

[45] Li Deng, Dong Yu, et al. Deep learning: methods and applications. Foundations and Trends® in Signal Processing, 7(3-4):197-387, 2014.

[46] Matt W Gardner and SR Dorling. Artificial neural networks (the multilayer perceptron) - a review of applications in the atmospheric sciences. Atmospheric environment, 32(14-15):2627-2636, 1998.

[47] Herbert Robbins and Sutton Monro. A stochastic approximation method. The annals of mathematical statistics, pages 400-407, 1951.

[48] Ilya Sutskever, James Martens, George Dahl, and Geoffrey Hinton. On the importance of initialization and momentum in deep learning. In International conference on machine learning, pages 1139-1147, 2013.

[49] John Duchi, Elad Hazan, and Yoram Singer. Adaptive subgradient methods for online learning and stochastic optimization. Journal of Machine Learning Research, 12(Jul):2121-2159, 2011.

[50] Tijmen Tieleman and Geoffrey Hinton. Lecture 6.5-rmsprop: Divide the gradient by a running average of its recent magnitude. COURSERA: Neural networks for machine learning, 4(2):26-31, 2012.

[51] Diederik P Kingma and Jimmy Ba. Adam: A method for stochastic optimization. arXiv preprint arXiv:1412.6980, 2014.

[52] Yoshua Bengio, Patrice Simard, Paolo Frasconi, et al. Learning long-term dependencies with gradient descent is difficult. IEEE transactions on neural networks, 5(2):157-166, 1994.

[53] Kaiming He, Xiangyu Zhang, Shaoqing Ren, and Jian Sun. Delving deep into rectifiers: Surpassing human-level performance on imagenet classification. In Proceedings of the IEEE international conference on computer vision, pages 1026-1034, 2015.

[54] Xavier Glorot and Yoshua Bengio. Understanding the difficulty of training deep feedforward neural networks. In Proceedings of the thirteenth international conference on artificial intelligence and statistics, pages 249-256, 2010.

[55] James S Bergstra, Rémi Bardenet, Yoshua Bengio, and Balázs Kégl. Algorithms for hyper-parameter optimization. In Advances in neural information processing systems, pages 2546-2554, 2011.

[56] James Bergstra and Yoshua Bengio. Random search for hyper-parameter optimization. Journal of Machine Learning Research, 13(Feb):281-305, 2012.

[57] Razvan Pascanu, Tomas Mikolov, and Yoshua Bengio. On the difficulty of training recurrent neural networks. In International conference on machine learning, pages 1310-1318, 2013.

[58] Sepp Hochreiter and Jürgen Schmidhuber. Long short-term memory. Neural computation, 9(8):1735$1780,1997$.

[59] Yonghui Wu, Mike Schuster, Zhifeng Chen, Quoc V Le, Mohammad Norouzi, Wolfgang Macherey, Maxim Krikun, Yuan Cao, Qin Gao, Klaus Macherey, et al. Google's neural machine translation system: Bridging the gap between human and machine translation. arXiv preprint arXiv:1609.08144, 2016.

[60] Klaus Greff, Rupesh K Srivastava, Jan Koutník, Bas R Steunebrink, and Jürgen Schmidhuber. Lstm: A search space odyssey. IEEE transactions on neural networks and learning systems, 28(10):22222232,2016

[61] Nils Reimers and Iryna Gurevych. Optimal hyperparameters for deep lstm-networks for sequence labeling tasks. arXiv preprint arXiv:1707.06799, 2017.

[62] Shuiwang Ji, Wei Xu, Ming Yang, and Kai Yu. 3d convolutional neural networks for human action recognition. IEEE transactions on pattern analysis and machine intelligence, 35(1):221-231, 2012.

[63] Christian Szegedy, Alexander Toshev, and Dumitru Erhan. Deep neural networks for object detection. In Advances in neural information processing systems, pages 2553-2561, 2013.

[64] Jonathan Long, Evan Shelhamer, and Trevor Darrell. Fully convolutional networks for semantic segmentation. In Proceedings of the IEEE conference on computer vision and pattern recognition, pages 3431-3440, 2015.

[65] Xueheng Qiu, Le Zhang, Ye Ren, P. Suganthan, and Gehan Amaratunga. Ensemble deep learning for regression and time series forecasting. In 2014 IEEE Symposium on Computational Intelligence in 
Ensemble Learning (CIEL), pages 1-6, 2014.

[66] Rafael Hrasko, André GC Pacheco, and Renato A Krohling. Time series prediction using restricted boltzmann machines and backpropagation. Procedia Computer Science, 55:990-999, 2015.

[67] Ruslan Salakhutdinov, Andriy Mnih, and Geoffrey Hinton. Restricted boltzmann machines for collaborative filtering. In Proceedings of the 24th international conference on Machine learning, pages 791-798. ACM, 2007.

[68] Yoshua Bengio. Deep learning of representations for unsupervised and transfer learning. In Proceedings of ICML workshop on unsupervised and transfer learning, pages 17-36, 2012.

[69] Abdel-rahman Mohamed, George Dahl, and Geoffrey Hinton. Deep belief networks for phone recognition. In Nips workshop on deep learning for speech recognition and related applications, volume 1, page 39. Vancouver, Canada, 2009.

[70] Honglak Lee, Roger Grosse, Rajesh Ranganath, and Andrew Y Ng. Convolutional deep belief networks for scalable unsupervised learning of hierarchical representations. In Proceedings of the 26th annual international conference on machine learning, pages 609-616. ACM, 2009.

[71] Laurens Van Der Maaten. Learning a parametric embedding by preserving local structure. In Artificial Intelligence and Statistics, pages 384-391, 2009.

[72] Chengwei Yao and Gencai Chen. Hyperparameters adaptation for restricted boltzmann machines based on free energy. In 2016 8th International Conference on Intelligent Human-Machine Systems and Cybernetics (IHMSC), volume 2, pages 243-248. IEEE, 2016.

[73] Miguel A Carreira-Perpinan and Geoffrey E Hinton. On contrastive divergence learning. In Aistats, volume 10, pages 33-40. Citeseer, 2005.

[74] Prasanna Tamilselvan and Pingfeng Wang. Failure diagnosis using deep belief learning based health state classification. Reliability Engineering \& System Safety, 115:124-135, 2013.

[75] Geoffrey E. Hinton, Simon Osindero, and Yee-Whye Teh. A fast learning algorithm for deep belief nets. Neural Computation, 18(7):1527-1554, 2006.

[76] Qinxue Meng, Daniel Catchpoole, David Skillicom, and Paul J Kennedy. Relational autoencoder for feature extraction. In 2017 International Joint Conference on Neural Networks (IJCNN), pages 364-371. IEEE, 2017.

[77] Pascal Vincent, Hugo Larochelle, Yoshua Bengio, and Pierre-Antoine Manzagol. Extracting and composing robust features with denoising autoencoders. In Proceedings of the 25th international conference on Machine learning, pages 1096-1103. ACM, 2008.

[78] Richard S Sutton and Andrew G Barto. Introduction to reinforcement learning, volume 135. MIT press Cambridge, 1998.

[79] Duy Nguyen-Tuong and Jan Peters. Model learning for robot control: a survey. Cognitive processing, 12(4):319-340, 2011.

[80] Eunsuk Chong, Chulwoo Han, and Frank C. Park. Deep learning networks for stock market analysis and prediction: Methodology, data representations, and case studies. Expert Systems with Applications, 83:187-205, October 2017.

[81] Kai Chen, Yi Zhou, and Fangyan Dai. A lstm-based method for stock returns prediction: A case study of china stock market. In 2015 IEEE International Conference on Big Data (Big Data). IEEE, October 2015.

[82] Eva Dezsi and Ioan Alin Nistor. Can deep machine learning outsmart the market? a comparison between econometric modelling and long- short term memory. Romanian Economic Business Review, 11(4.1):54-73, December 2016.

[83] A.J.P. Samarawickrama and T.G.I. Fernando. A recurrent neural network approach in predicting daily stock prices an application to the sri lankan stock market. In 2017 IEEE International Conference on Industrial and Information Systems (ICIIS). IEEE, December 2017.

[84] M Hiransha, E.A. Gopalakrishnan, Vijay Krishna Menon, and K.P. Soman. Nse stock market prediction using deep-learning models. Procedia Computer Science, 132:1351-1362, 2018.

[85] Sreelekshmy Selvin, R Vinayakumar, E. A Gopalakrishnan, Vijay Krishna Menon, and K. P. Soman. Stock price prediction using lstm, rnn and cnn-sliding window model. In 2017 International Conference 
on Advances in Computing, Communications and Informatics (ICACCI). IEEE, September 2017.

[86] Sang Il Lee and Seong Joon Yoo. Threshold-based portfolio: the role of the threshold and its applications. The Journal of Supercomputing, September 2018.

[87] Xiumin Li, Lin Yang, Fangzheng Xue, and Hongjun Zhou. Time series prediction of stock price using deep belief networks with intrinsic plasticity. In 2017 29th Chinese Control And Decision Conference $(C C D C)$. IEEE, May 2017.

[88] Lin Chen, Zhilin Qiao, Minggang Wang, Chao Wang, Ruijin Du, and Harry Eugene Stanley. Which artificial intelligence algorithm better predicts the chinese stock market? IEEE Access, 6:48625-48633, 2018.

[89] Christopher Krauss, Xuan Anh Do, and Nicolas Huck. Deep neural networks, gradient-boosted trees, random forests: Statistical arbitrage on the s\&p 500. European Journal of Operational Research, 259 (2):689-702, June 2017.

[90] Rohitash Chandra and Shelvin Chand. Evaluation of co-evolutionary neural network architectures for time series prediction with mobile application in finance. Applied Soft Computing, 49:462-473, December 2016.

[91] Shuanglong Liu, Chao Zhang, and Jinwen Ma. Cnn-lstm neural network model for quantitative strategy analysis in stock markets. In Neural Information Processing, pages 198-206. Springer International Publishing, 2017.

[92] J. B. Heaton, N. G. Polson, and J. H. Witte. Deep learning in finance, 2016.

[93] Bilberto Batres-Estrada. Deep learning for multivariate financial time series. Master's thesis, KTH, Mathematical Statistics, 2015.

[94] Zhaozheng Yuan, Ruixun Zhang, and Xiuli Shao. Deep and wide neural networks on multiple sets of temporal data with correlation. In Proceedings of the 2018 International Conference on Computing and Data Engineering - ICCDE 2018. ACM Press, 2018.

[95] Liheng Zhang, Charu Aggarwal, and Guo-Jun Qi. Stock price prediction via discovering multifrequency trading patterns. In Proceedings of the 23rd ACM SIGKDD International Conference on Knowledge Discovery and Data Mining - KDD17. ACM Press, 2017.

[96] Masaya Abe and Hideki Nakayama. Deep learning for forecasting stock returns in the cross-section. In Advances in Knowledge Discovery and Data Mining, pages 273-284. Springer International Publishing, 2018.

[97] Guanhao Feng, Jingyu He, and Nicholas G. Polson. Deep learning for predicting asset returns, 2018.

[98] Jianqing Fan, Lingzhou Xue, and Jiawei Yao. Sufficient forecasting using factor models. SSRN Electronic Journal, 2014.

[99] Mathias Kraus and Stefan Feuerriegel. Decision support from financial disclosures with deep neural networks and transfer learning. Decision Support Systems, 104:38-48, December 2017.

[100] Shotaro Minami. Predicting equity price with corporate action events using lstm-rnn. Journal of Mathematical Finance, 08(01):58-63, 2018.

[101] Xiaolin Zhang and Ying Tan. Deep stock ranker: A lstm neural network model for stock selection. In Data Mining and Big Data, pages 614-623. Springer International Publishing, 2018.

[102] Qun Zhuge, Lingyu Xu, and Gaowei Zhang. Lstm neural network with emotional analysis for prediction of stock price. 2017.

[103] Ryo Akita, Akira Yoshihara, Takashi Matsubara, and Kuniaki Uehara. Deep learning for stock prediction using numerical and textual information. In 2016 IEEE/ACIS 15th International Conference on Computer and Information Science (ICIS). IEEE, June 2016.

[104] A. Ozbayoglu. Neural based technical analysis in stock market forecasting. In Intelligent Engineering Systems Through Artificial Neural Networks, Volume 17, pages 261-266. ASME, 2007.

[105] Kaustubh Khare, Omkar Darekar, Prafull Gupta, and V. Z. Attar. Short term stock price prediction using deep learning. In 2017 2nd IEEE International Conference on Recent Trends in Electronics, Information \& Communication Technology (RTEICT). IEEE, May 2017.

[106] Xingyu Zhou, Zhisong Pan, Guyu Hu, Siqi Tang, and Cheng Zhao. Stock market prediction on highfrequency data using generative adversarial nets. Mathematical Problems in Engineering, 2018:1-11, 
2018.

[107] Ritika Singh and Shashi Srivastava. Stock prediction using deep learning. Multimedia Tools and Applications, 76(18):18569-18584, December 2016.

[108] Sercan Karaoglu and Ugur Arpaci. A deep learning approach for optimization of systematic signal detection in financial trading systems with big data. International Journal of Intelligent Systems and Applications in Engineering, SpecialIssue(SpecialIssue):31-36, July 2017.

[109] Bo Zhou. Deep learning and the cross-section of stock returns: Neural networks combining price and fundamental information. SSRN Electronic Journal, 2018.

[110] Narek Abroyan and. Neural networks for financial market risk classification. Frontiers in Signal Processing, 1(2), August 2017.

[111] Google. System and method for computer managed funds to outperform benchmarks.

[112] Dat Thanh Tran, Martin Magris, Juho Kanniainen, Moncef Gabbouj, and Alexandros Iosifidis. Tensor representation in high-frequency financial data for price change prediction. In 2017 IEEE Symposium Series on Computational Intelligence (SSCI). IEEE, November 2017.

[113] Guanhao Feng, Nicholas G. Polson, and Jianeng Xu. Deep factor alpha, 2018.

[114] Xiao Ding, Yue Zhang, Ting Liu, and Junwen Duan. Deep learning for event-driven stock prediction. In Proceedings of the 24th International Conference on Artificial Intelligence, IJCAI'15, pages 23272333. AAAI Press, 2015.

[115] Manuel R. Vargas, Beatriz S. L. P. de Lima, and Alexandre G. Evsukoff. Deep learning for stock market prediction from financial news articles. In 2017 IEEE International Conference on Computational Intelligence and Virtual Environments for Measurement Systems and Applications (CIVEMSA). IEEE, June 2017.

[116] Che-Yu Lee and Von-Wun Soo. Predict stock price with financial news based on recurrent convolutional neural networks. In 2017 Conference on Technologies and Applications of Artificial Intelligence (TAAI). IEEE, December 2017.

[117] Hitoshi Iwasaki and Ying Chen. Topic sentiment asset pricing with dnn supervised learning. SSRN Electronic Journal, 2018.

[118] Sushree Das, Ranjan Kumar Behera, Mukesh Kumar, and Santanu Kumar Rath. Real-time sentiment analysis of twitter streaming data for stock prediction. Procedia Computer Science, 132:956-964, 2018.

[119] Jiahong Li, Hui Bu, and Junjie Wu. Sentiment-aware stock market prediction: A deep learning method. In 2017 International Conference on Service Systems and Service Management. IEEE, June 2017.

[120] Zhongshengz. Measuring financial crisis index for risk warning through analysis of social network. Master's thesis, 2018.

[121] Janderson B. Nascimento and Marco Cristo. The impact of structured event embeddings on scalable stock forecasting models. In Proceedings of the 21st Brazilian Symposium on Multimedia and the Web - WebMedia15. ACM Press, 2015.

[122] Songqiao Han, Xiaoling Hao, and Hailiang Huang. An event-extraction approach for business analysis from online chinese news. Electronic Commerce Research and Applications, 28:244-260, March 2018.

[123] Wei Bao, Jun Yue, and Yulei Rao. A deep learning framework for financial time series using stacked autoencoders and long-short term memory. PLOS ONE, 12(7):e0180944, July 2017.

[124] A.K. Parida, R. Bisoi, and P.K. Dash. Chebyshev polynomial functions based locally recurrent neurofuzzy information system for prediction of financial and energy market data. The Journal of Finance and Data Science, 2(3):202-223, September 2016.

[125] Thomas Fischer and Christopher Krauss. Deep learning with long short-term memory networks for financial market predictions. European Journal of Operational Research, 270(2):654-669, October 2018.

[126] Philip Widegren. Deep learning-based forecasting of financial assets. Master's thesis, KTH, Mathematical Statistics, 2017.

[127] Anastasia Borovykh, Sander Bohte, and Cornelis W. Oosterlee. Dilated convolutional neural networks for time series forecasting. Journal of Computational Finance, October 2018. 
[128] Khaled A. Althelaya, El-Sayed M. El-Alfy, and Salahadin Mohammed. Evaluation of bidirectional lstm for short-and long-term stock market prediction. In 2018 9th International Conference on Information and Communication Systems (ICICS). IEEE, April 2018.

[129] Alexiei Dingli and Karl Sant Fournier. Financial time series forecasting-a deep learning approach. Int. J. Mach. Learn. Comput, 7(5):118-122, 2017.

[130] Ajit Kumar Rout, P.K. Dash, Rajashree Dash, and Ranjeeta Bisoi. Forecasting financial time series using a low complexity recurrent neural network and evolutionary learning approach. Journal of King Saud University - Computer and Information Sciences, 29(4):536-552, October 2017.

[131] Gyeeun Jeong and Ha Young Kim. Improving financial trading decisions using deep q-learning: Predicting the number of shares, action strategies, and transfer learning. Expert Systems with Applications, 117:125-138, March 2019 .

[132] Yujin Baek and Ha Young Kim. Modaugnet: A new forecasting framework for stock market index value with an overfitting prevention lstm module and a prediction lstm module. Expert Systems with Applications, 113:457-480, December 2018.

[133] Magnus Hansson. On stock return prediction with lstm networks. 2017.

[134] Aaron Elliot and Cheng Hua Hsu. Time series prediction : Predicting stock price, 2017.

[135] Zhixi Li and Vincent Tam. Combining the real-time wavelet denoising and long-short-term-memory neural network for predicting stock indexes. In 2017 IEEE Symposium Series on Computational Intelligence (SSCI). IEEE, November 2017.

[136] Sima Siami-Namini and Akbar Siami Namin. Forecasting economics and financial time series: Arima vs. lstm, 2018.

[137] Tsung-Jung Hsieh, Hsiao-Fen Hsiao, and Wei-Chang Yeh. Forecasting stock markets using wavelet transforms and recurrent neural networks: An integrated system based on artificial bee colony algorithm. Applied Soft Computing, 11(2):2510-2525, March 2011.

[138] Luna M. Zhang. Genetic deep neural networks using different activation functions for financial data mining. In 2015 IEEE International Conference on Big Data (Big Data). IEEE, October 2015.

[139] Stelios D. Bekiros. Irrational fads, short-term memory emulation, and asset predictability. Review of Financial Economics, 22(4):213-219, November 2013.

[140] Xiongwen Pang, Yanqiang Zhou, Pan Wang, Weiwei Lin, and Victor Chang. An innovative neural network approach for stock market prediction. The Journal of Supercomputing, January 2018.

[141] Yue Deng, Feng Bao, Youyong Kong, Zhiquan Ren, and Qionghai Dai. Deep direct reinforcement learning for financial signal representation and trading. IEEE Transactions on Neural Networks and Learning Systems, 28(3):653-664, March 2017.

[142] Bing Yang, Zi-Jia Gong, and Wenqi Yang. Stock market index prediction using deep neural network ensemble. In 2017 36th Chinese Control Conference (CCC). IEEE, July 2017.

[143] Oussama Lachiheb and Mohamed Salah Gouider. A hierarchical deep neural network design for stock returns prediction. Procedia Computer Science, 126:264-272, 2018.

[144] Bang Xiang Yong, Mohd Rozaini Abdul Rahim, and Ahmad Shahidan Abdullah. A stock market trading system using deep neural network. In Communications in Computer and Information Science, pages 356-364. Springer Singapore, 2017.

[145] Serdar Yümlü, Fikret S. Gürgen, and Nesrin Okay. A comparison of global, recurrent and smoothedpiecewise neural models for istanbul stock exchange (ise) prediction. Pattern Recognition Letters, 26 (13):2093-2103, October 2005.

[146] Hongju Yan and Hongbing Ouyang. Financial time series prediction based on deep learning. Wireless Personal Communications, 102(2):683-700, December 2017.

[147] Takahashi. Long memory and predictability in financial markets. Annual Conference of the Japanese Society for Artificial Intelligence, 2017.

[148] Melike Bildirici, Elçin A. Alp, and Özgür Ö. Ersin. Tar-cointegration neural network model: An empirical analysis of exchange rates and stock returns. Expert Systems with Applications, 37(1):2-11, January 2010.

[149] Ioannis Psaradellis and Georgios Sermpinis. Modelling and trading the u.s. implied volatility indices. 
evidence from the vix, vxn and vxd indices. International Journal of Forecasting, 32(4):1268-1283, October 2016.

[150] Jiaqi Chen, Wenbo Wu, and Michael Tindall. Hedge fund return prediction and fund selection: A machine-learning approach. Occasional Papers 16-4, Federal Reserve Bank of Dallas, November 2016.

[151] Marios Mourelatos, Christos Alexakos, Thomas Amorgianiotis, and Spiridon Likothanassis. Financial indices modelling and trading utilizing deep learning techniques: The athens se ftse/ase large cap use case. In 2018 Innovations in Intelligent Systems and Applications (INISTA). IEEE, July 2018.

[152] Yuzhou Chen, Junji Wu, and Hui Bu. Stock market embedding and prediction: A deep learning method. In 2018 15th International Conference on Service Systems and Service Management (ICSSSM). IEEE, July 2018.

[153] Weiyu Si, Jinke Li, Peng Ding, and Ruonan Rao. A multi-objective deep reinforcement learning approach for stock index future's intraday trading. In 2017 10th International Symposium on Computational Intelligence and Design (ISCID). IEEE, December 2017.

[154] Weiling Chen, Chai Kiat Yeo, Chiew Tong Lau, and Bu Sung Lee. Leveraging social media news to predict stock index movement using rnn-boost. Data $\mathscr{E}$ Knowledge Engineering, August 2018.

[155] Matthew Francis Dixon, Diego Klabjan, and Jin Hoon Bang. Classification-based financial markets prediction using deep neural networks. SSRN Electronic Journal, 2016.

[156] Fernando Sánchez Lasheras, Francisco Javier de Cos Juez, Ana Suárez Sánchez, Alicja Krzemień, and Pedro Riesgo Fernández. Forecasting the comex copper spot price by means of neural networks and arima models. Resources Policy, 45:37-43, September 2015.

[157] Yang Zhao, Jianping Li, and Lean Yu. A deep learning ensemble approach for crude oil price forecasting. Energy Economics, 66:9-16, August 2017.

[158] Yanhui Chen, Kaijian He, and Geoffrey K.F. Tso. Forecasting crude oil prices: a deep learning based model. Procedia Computer Science, 122:300-307, 2017.

[159] Jonathan Doering, Michael Fairbank, and Sheri Markose. Convolutional neural networks applied to high-frequency market microstructure forecasting. In 2017 9th Computer Science and Electronic Engineering (CEEC). IEEE, September 2017.

[160] P. Tino, C. Schittenkopf, and G. Dorffner. Financial volatility trading using recurrent neural networks. IEEE Transactions on Neural Networks, 12(4):865-874, July 2001.

[161] Ruoxuan Xiong, Eric P. Nichols, and Yuan Shen. Deep learning stock volatility with google domestic trends, 2015.

[162] Yu-Long Zhou, Ren-Jie Han, Qian Xu, and Wei-Ke Zhang. Long short-term memory networks for csi300 volatility prediction with baidu search volume. 2018.

[163] Ha Young Kim and Chang Hyun Won. Forecasting the volatility of stock price index: A hybrid model integrating lstm with multiple garch-type models. Expert Systems with Applications, 103:2537, August 2018.

[164] Nikolay Nikolaev, Peter Tino, and Evgueni Smirnov. Time-dependent series variance learning with recurrent mixture density networks. Neurocomputing, 122:501-512, December 2013.

[165] Campbell R. Harvey. Forecasts of economic growth from the bond and stock markets. Financial Analysts Journal, 45(5):38-45, 1989.

[166] Daniele Bianchi, Matthias Büchner, and Andrea Tamoni. Bond risk premia with machine learning. SSRN Electronic Journal, September 2018.

[167] Venketas Warren. Forex market size: A traders advantage, 2019.

[168] Ren Zhang, Furao Shen, and Jinxi Zhao. A model with fuzzy granulation and deep belief networks for exchange rate forecasting. In 2014 International Joint Conference on Neural Networks (IJCNN). IEEE, July 2014.

[169] Jing Chao, Furao Shen, and Jinxi Zhao. Forecasting exchange rate with deep belief networks. In The 2011 International Joint Conference on Neural Networks. IEEE, July 2011.

[170] Jing Zheng, Xiao Fu, and Guijun Zhang. Research on exchange rate forecasting based on deep belief network. Neural Computing and Applications, May 2017.

[171] Furao Shen, Jing Chao, and Jinxi Zhao. Forecasting exchange rate using deep belief networks and 
conjugate gradient method. Neurocomputing, 167:243-253, November 2015.

[172] Hua Shen and Xun Liang. A time series forecasting model based on deep learning integrated algorithm with stacked autoencoders and svr for fx prediction. In ICANN, 2016.

[173] Georgios Sermpinis, Jason Laws, Andreas Karathanasopoulos, and Christian L. Dunis. Forecasting and trading the eur/usd exchange rate with gene expression and psi sigma neural networks. Expert Systems with Applications, 39(10):8865-8877, August 2012.

[174] Georgios Sermpinis, Christian Dunis, Jason Laws, and Charalampos Stasinakis. Forecasting and trading the eur/usd exchange rate with stochastic neural network combination and time-varying leverage. Decision Support Systems, 54(1):316-329, December 2012.

[175] Georgios Sermpinis, Charalampos Stasinakis, and Christian Dunis. Stochastic and genetic neural network combinations in trading and hybrid time-varying leverage effects. Journal of International Financial Markets, Institutions and Money, 30:21-54, May 2014.

[176] Bo SUN and Chi XIE. Rmb exchange rate forecasting in the context of the financial crisis. Systems Engineering - Theory \& Practice, 29(12):53-64, December 2009.

[177] Nijolè Maknickienė and Algirdas Maknickas. Financial market prediction system with evolino neural network and deplhi method. Journal of Business Economics and Management, 14(2):403-413, May 2013.

[178] Nijole Maknickiene, Aleksandras Vytautas Rutkauskas, and Algirdas Maknickas. Investigation of financial market prediction by recurrent neural network. 2014.

[179] Luca Di Persio and Oleksandr Honchar. Artificial neural networks approach to the forecast of stock market price movements. International Journal of Economics and Management Systems, (1):158-162, 2016.

[180] Jerzy Korczak and Marcin Hernes. Deep learning for financial time series forecasting in a-trader system. In Proceedings of the 2017 Federated Conference on Computer Science and Information Systems. IEEE, September 2017.

[181] Gonçalo Duarte Lima Freire Lopes. Deep learning for market forecasts. 2018.

[182] Sean McNally, Jason Roche, and Simon Caton. Predicting the price of bitcoin using machine learning. In 2018 26th Euromicro International Conference on Parallel, Distributed and Network-based Processing (PDP). IEEE, March 2018.

[183] Sanjiv Das, Karthik Mokashi, and Robbie Culkin. Are markets truly efficient? experiments using deep learning algorithms for market movement prediction. Algorithms, 11(9):138, September 2018.

[184] Ariel Navon and Yosi Keller. Financial time series prediction using deep learning, 2017.

[185] E.W. Saad, D.V. Prokhorov, and D.C. Wunsch. Comparative study of stock trend prediction using time delay, recurrent and probabilistic neural networks. IEEE Transactions on Neural Networks, 9 (6):1456-1470, 1998.

[186] Luca Di Persio and Oleksandr Honchar. Recurrent neural networks approach to the financial forecast of google assets. International Journal of Mathematics and Computers in Simulation, 11:713, 2017.

[187] Guizhu Shen, Qingping Tan, Haoyu Zhang, Ping Zeng, and Jianjun Xu. Deep learning with gated recurrent unit networks for financial sequence predictions. Procedia Computer Science, 131:895-903, 2018.

[188] Jou-Fan Chen, Wei-Lun Chen, Chun-Ping Huang, Szu-Hao Huang, and An-Pin Chen. Financial timeseries data analysis using deep convolutional neural networks. In 2016 7th International Conference on Cloud Computing and Big Data (CCBD). IEEE, November 2016.

[189] Omer Berat Sezer and Ahmet Murat Ozbayoglu. Financial trading model with stock bar chart image time series with deep convolutional neural networks. arXiv preprint arXiv:1903.04610, 2019.

[190] Feng Zhou, Hao min Zhou, Zhihua Yang, and Lihua Yang. Emd2fnn: A strategy combining empirical mode decomposition and factorization machine based neural network for stock market trend prediction. Expert Systems with Applications, 115:136-151, 2019.

[191] Kristiina Ausmees, Slobodan Milovanovic, Fredrik Wrede, and Afshin Zafari. Taming deep belief networks. 2017.

[192] Kamran Raza. Prediction of stock market performance by using machine learning techniques. In 2017 
International Conference on Innovations in Electrical Engineering and Computational Technologies (ICIEECT). IEEE, April 2017.

[193] Omer Berat Sezer, Murat Ozbayoglu, and Erdogan Dogdu. A deep neural-network based stock trading system based on evolutionary optimized technical analysis parameters. Procedia Computer Science, 114:473-480, 2017.

[194] Qiubin Liang, Wenge Rong, Jiayi Zhang, Jingshuang Liu, and Zhang Xiong. Restricted boltzmann machine based stock market trend prediction. In 2017 International Joint Conference on Neural Networks (IJCNN). IEEE, May 2017.

[195] Luigi Troiano, Elena Mejuto Villa, and Vincenzo Loia. Replicating a trading strategy by means of lstm for financial industry applications. IEEE Transactions on Industrial Informatics, 14(7):32263234 , July 2018.

[196] David M. Q. Nelson, Adriano C. M. Pereira, and Renato A. de Oliveira. Stock markets price movement prediction with lstm neural networks. In 2017 International Joint Conference on Neural Networks (IJCNN). IEEE, May 2017.

[197] Yuan Song and Yingnian Wu. Stock trend prediction: Based on machine learning methods. Master's thesis, 2018.

[198] M. Ugur Gudelek, S. Arda Boluk, and A. Murat Ozbayoglu. A deep learning based stock trading model with 2-d cnn trend detection. In 2017 IEEE Symposium Series on Computational Intelligence (SSCI). IEEE, November 2017.

[199] Omer Berat Sezer and Ahmet Murat Ozbayoglu. Algorithmic financial trading with deep convolutional neural networks: Time series to image conversion approach. Applied Soft Computing, 70:525-538, September 2018.

[200] Hakan Gunduz, Yusuf Yaslan, and Zehra Cataltepe. Intraday prediction of borsa istanbul using convolutional neural networks and feature correlations. Knowledge-Based Systems, 137:138-148, December 2017.

[201] Yifu Huang, Kai Huang, Yang Wang, Hao Zhang, Jihong Guan, and Shuigeng Zhou. Exploiting twitter moods to boost financial trend prediction based on deep network models. In Intelligent Computing Methodologies, pages 449-460. Springer International Publishing, 2016.

[202] Yangtuo Peng and Hui Jiang. Leverage financial news to predict stock price movements using word embeddings and deep neural networks. In Proceedings of the 2016 Conference of the North American Chapter of the Association for Computational Linguistics: Human Language Technologies. Association for Computational Linguistics, 2016.

[203] Huy D. Huynh, L. Minh Dang, and Duc Duong. A new model for stock price movements prediction using deep neural network. In Proceedings of the Eighth International Symposium on Information and Communication Technology - SoICT 2017. ACM Press, 2017.

[204] L. Minh Dang, Abolghasem Sadeghi-Niaraki, Huy D. Huynh, Kyungbok Min, and Hyeonjoon Moon. Deep learning approach for short-term stock trends prediction based on two-stream gated recurrent unit network. IEEE Access, pages 1-1, 2018.

[205] Ishan Verma, Lipika Dey, and Hardik Meisheri. Detecting, quantifying and accessing impact of news events on indian stock indices. In Proceedings of the International Conference on Web Intelligence WI17. ACM Press, 2017.

[206] Leonardo dos Santos Pinheiro and Mark Dras. Stock market prediction with deep learning: A character-based neural language model for event-based trading. In Proceedings of the Australasian Language Technology Association Workshop 2017, pages 6-15, 2017.

[207] Jordan Prosky, Xingyou Song, Andrew Tan, and Michael Zhao. Sentiment predictability for stocks. CoRR, abs/1712.05785, 2017.

[208] Yang Liu, Qingguo Zeng, Huanrui Yang, and Adrian Carrio. Stock price movement prediction from financial news with deep learning and knowledge graph embedding. In Knowledge Management and Acquisition for Intelligent Systems, pages 102-113. Springer International Publishing, 2018.

[209] Akira Yoshihara, Kazuki Fujikawa, Kazuhiro Seki, and Kuniaki Uehara. Predicting stock market trends by recurrent deep neural networks. In Lecture Notes in Computer Science, pages 759-769. 
Springer International Publishing, 2014.

[210] Lei Shi, Zhiyang Teng, Le Wang, Yue Zhang, and Alexander Binder. Deepclue: Visual interpretation of text-based deep stock prediction. IEEE Transactions on Knowledge and Data Engineering, pages $1-1,2018$.

[211] Xi Zhang, Yunjia Zhang, Senzhang Wang, Yuntao Yao, Binxing Fang, and Philip S. Yu. Improving stock market prediction via heterogeneous information fusion. Knowledge-Based Systems, 143:236-247, March 2018.

[212] Ziniu Hu, Weiqing Liu, Jiang Bian, Xuanzhe Liu, and Tie-Yan Liu. Listening to chaotic whispers: A deep learning framework for news-oriented stock trend prediction. In Proceedings of the Eleventh ACM International Conference on Web Search and Data Mining, WSDM '18, pages 261-269, New York, NY, USA, 2018. ACM.

[213] Qili Wang, Wei Xu, and Han Zheng. Combining the wisdom of crowds and technical analysis for financial market prediction using deep random subspace ensembles. Neurocomputing, 299:51-61, July 2018.

[214] Takashi Matsubara, Ryo Akita, and Kuniaki Uehara. Stock price prediction by deep neural generative model of news articles. IEICE Transactions on Information and Systems, E101.D(4):901-908, 2018.

[215] Xiaodong Li, Jingjing Cao, and Zhaoqing Pan. Market impact analysis via deep learned architectures. Neural Computing and Applications, March 2018.

[216] Avraam Tsantekidis, Nikolaos Passalis, Anastasios Tefas, Juho Kanniainen, Moncef Gabbouj, and Alexandros Iosifidis. Using deep learning to detect price change indications in financial markets. In 2017 25th European Signal Processing Conference (EUSIPCO). IEEE, August 2017.

[217] Justin Sirignano and Rama Cont. Universal features of price formation in financial markets: Perspectives from deep learning. SSRN Electronic Journal, 2018.

[218] Przemyslaw Buczkowski. Predicting stock trends based on expert recommendations using gru/lstm neural networks. In Lecture Notes in Computer Science, pages 708-717. Springer International Publishing, 2017.

[219] Avraam Tsantekidis, Nikolaos Passalis, Anastasios Tefas, Juho Kanniainen, Moncef Gabbouj, and Alexandros Iosifidis. Forecasting stock prices from the limit order book using convolutional neural networks. In 2017 IEEE 19th Conference on Business Informatics (CBI). IEEE, July 2017.

[220] Thomas G Thomas Günter Fischer, Christopher Krauss, and Alexander Deinert. Statistical arbitrage in cryptocurrency markets. Journal of Risk and Financial Management, 12, 2019. 